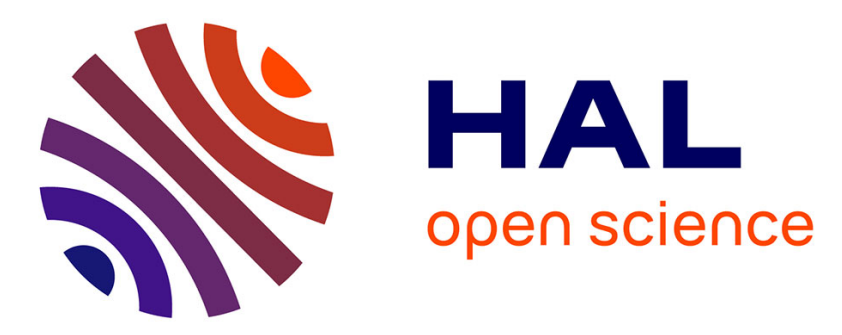

\title{
Delayed stabilization of the Korteweg-de Vries equation on a Star-shaped network
}

\author{
Hugo Parada, Emmanuelle Crépeau, Christophe Prieur
}

\section{To cite this version:}

Hugo Parada, Emmanuelle Crépeau, Christophe Prieur. Delayed stabilization of the Korteweg-de Vries equation on a Star-shaped network. Mathematics of Control, Signals, and Systems, 2022, 34 (3), pp.559-605. 10.1007/s00498-022-00319-0 . hal-03177446

\section{HAL Id: hal-03177446 \\ https://hal.science/hal-03177446}

Submitted on 23 Mar 2021

HAL is a multi-disciplinary open access archive for the deposit and dissemination of scientific research documents, whether they are published or not. The documents may come from teaching and research institutions in France or abroad, or from public or private research centers.
L'archive ouverte pluridisciplinaire HAL, est destinée au dépôt et à la diffusion de documents scientifiques de niveau recherche, publiés ou non, émanant des établissements d'enseignement et de recherche français ou étrangers, des laboratoires publics ou privés. 


\title{
Delayed stabilization of the Korteweg-de Vries equation on a Star-shaped network
}

\author{
Hugo Parada • Emmanuelle Crépeau • \\ Christophe Prieur
}

Received: date / Accepted: date

\begin{abstract}
In this work we deal with the exponential stability of the nonlinear Kortewegde Vries $(\mathrm{KdV})$ equation on a finite star-shaped network in the presence of delayed internal feedback. We start by proving the well-posedness of the system and some regularity results. Then we state an exponential stabilization result using a Lyapunov function by imposing small initial data and a restriction over the lengths. In this part also, we are able to obtain an explicit expression for the rate of decay. Then we prove the exponential stability of the solutions without restriction on the lengths and for small initial data, this result is based on an observability inequality. After that, we obtain a semi-global stabilization result working directly with the nonlinear system. Next we study the case where it may happen that a control domain with delay is outside of the control domain without delay. In that case, we obtain also a local exponential stabilization result. Finally, we present some numerical simulations in order to illustrate the stabilization.
\end{abstract}

Keywords $\mathrm{KdV}$ equation · delay · stabilization · Star-Shaped Network

Mathematics Subject Classification (2010) $35 \mathrm{Q} 53 \cdot 35 \mathrm{~B} 35 \cdot 35 \mathrm{R} 02$

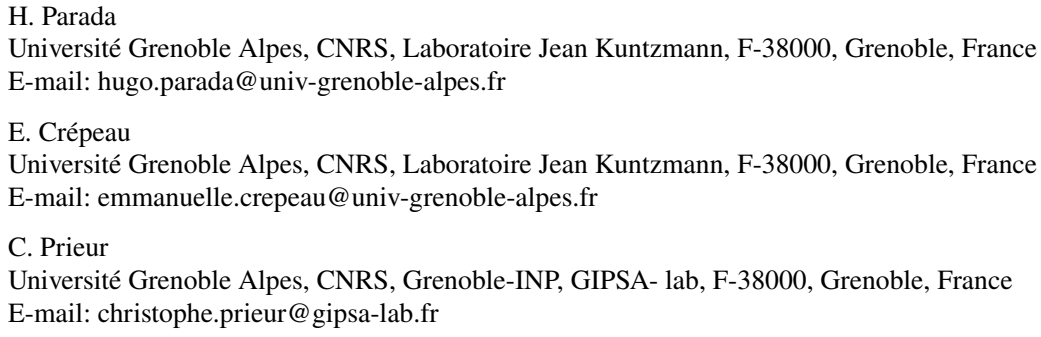

This work is supported by the French National Research Agency in the framework of the "Investissements d'avenir" program (ANR-15-IDEX-02). 


\section{Introduction}

The Korteweg-de Vries (KdV) equation $u_{t}+u_{x}+u_{x x x}+u u_{x}=0$ was introduced in [8] to model the propagation of long water waves in a channel. It has been widely studied in the last years, in particular its controllability and stabilization properties, see [3, 19] for a complete introduction to those problems.

From the stabilization point of view, we can refer to the work [22] where the boundary exponential stabilization problem was studied in the bounded spatial domain $x \in(0,1)$. It is well known that the length $L$ of the spatial domain plays an important role in the stabilization and controllability properties of the $\mathrm{KdV}$ equation. For example if $L=2 \pi$ it is possible to find a solution of the linearization around 0 of $\mathrm{KdV}$ $(u(t, x)=1-\cos (x))$ which has constant energy. More generally if $L \in \mathcal{N}$ where $\mathcal{N}$ is called the set of critical lengths defined by

$$
\mathcal{N}=\left\{2 \pi \sqrt{\frac{k^{2}+k l+l^{2}}{3}}, k, l \in \mathbb{N}^{*}\right\}
$$

we can find suitable initial data such that the solution of the linear KdV equation has constant energy. In the case of internal stabilization it is proven in [17, 15] that for any critical length by adding a localized damping we reach the local exponential stability for the nonlinear $\mathrm{KdV}$ equation.

Adding a delay term allows to study the action of a device in a more real-life setting. It is known that even the presence of small delays in internal feedback could destabilize a system, see for example [6]. In the works [2] and [21] the problem of robustness with respect to time delay for a $\mathrm{KdV}$ equation was studied with boundary and internal stabilization respectively. Our contribution to this work is to study the stabilization of a KdV equation posed on a Star Network in presence of internal time delays. With respect to the KdV equation on Networks the first work introducing this system was [1] where the stabilization and controllability problems were studied and after that the boundary controllabilty results were improved in [4].

In this work we are interested in the stability properties of the Korteweg-de Vries equation with internal input delay posed on a Star-Shaped Network. Let $K=\left\{k_{j}: 1 \leq\right.$ $j \leq N\}$ be the set of the edges of a network $\mathcal{T}$ described as the intervals $\left[0, \ell_{j}\right]$ with $\ell_{j}>0$ for $j=1, \cdots N$, the network $\mathcal{T}$ is defined by

$$
\mathcal{T}=\bigcup_{j=1}^{N} k_{j}
$$




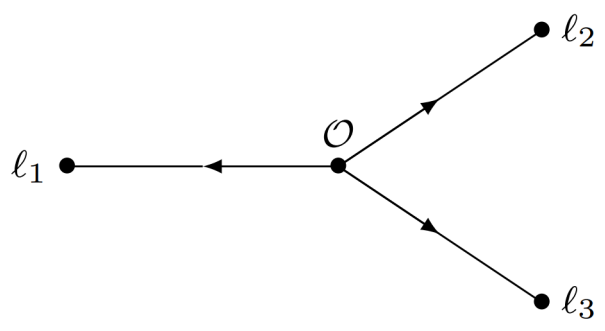

Fig. 1 Star Shaped Network for $N=3$.

Specifically we are going to consider the next evolution problem for the $\mathrm{KdV}$ equation with internal input delay on each edge.

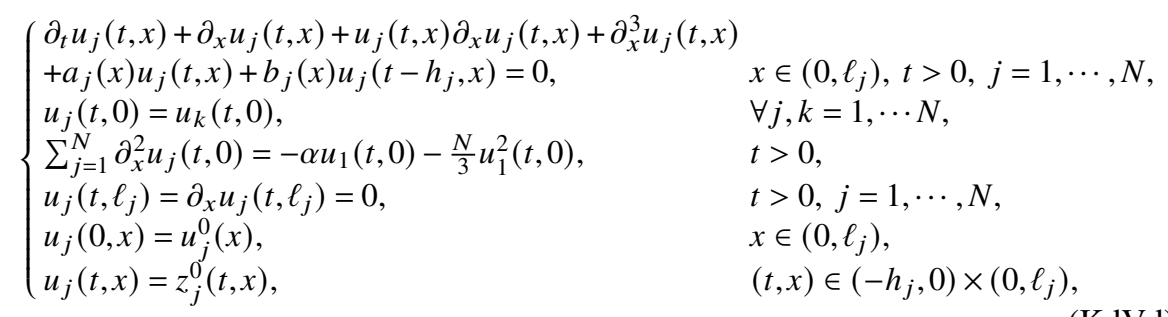

where $\alpha \geq \frac{N}{2}$ and for all $j=1, \cdots, N, h_{j}>0$ is the time delay on the edge $j, a_{j}$, $b_{j} \in L^{\infty}\left(0, \ell_{j}\right)$ are non-negative and supp $b_{j}=\omega_{j}$ is a nonempty, open subset of $\left(0, \ell_{j}\right)$ such that

$$
b_{j}(x) \geq b_{0}>0, \text { a.e on } \omega_{j},
$$

there exists $c_{0}>0$, such that $b_{j}(x)+c_{0} \leq a_{j}(x), \forall x \in \omega_{j}$.

The condition $\alpha>\frac{N}{2}$ was firstly introduced in [1] in order to have a decreasing energy, the case $\alpha=\frac{N}{2}$ was studied in [4] from a controllability point of view. In our work we consider $\alpha>\frac{N}{2}$ in some cases and $\alpha \geq \frac{N}{2}$ in others. The conditions over the damped terms with and without delay (1.1)- $(1.2)$ are the analogues of the conditions (1.2) - (1.3) presented in [21], similar conditions over the weight of the feedback with and without delay can be founded in [2, 13, 12].

In order to study this system we need first a proper functional setting. We define the following spaces

$$
H_{r}^{s}\left(0, \ell_{j}\right)=\left\{v \in H^{s}\left(0, \ell_{j}\right),\left(\frac{\mathrm{d}}{\mathrm{d} x}\right)^{i-1} v\left(\ell_{j}\right)=0,1 \leq i \leq s\right\}, s=1,2,
$$

where the index $r$ is related to the null right boundary conditions. The space $\mathbb{H}_{e}^{S}(\mathcal{T})$ will be the cartesian product of $H_{r}^{s}\left(0, \ell_{j}\right)$ including the continuity condition on the central node $\left(u_{j}(0)=u_{k}(0), \forall j, k=1, \cdots, N\right)$

$$
\mathbb{H}_{e}^{s}(\mathcal{T})=\left\{\underline{u}=\left(u_{1}, \cdots, u_{N}\right) \in \prod_{j=1}^{N} H_{r}^{s}\left(0, \ell_{j}\right), u_{j}(0)=u_{k}(0), \forall j, k=1, \cdots, N\right\}, s=1,2
$$


and

$$
\|\underline{u}\|_{\mathbb{H}_{e}^{1}(\mathcal{T})}^{2}=\sum_{j=1}^{N}\left\|u_{j}\right\|_{H^{1}\left(0, \ell_{j}\right)}^{2}
$$

where the index $e$ indicates that each edge belongs to $H_{r}^{s}\left(0, \ell_{j}\right)$.

$$
\mathbb{L}^{2}(\mathcal{T})=\prod_{j=1}^{N} L^{2}\left(0, \ell_{j}\right), \quad \mathbb{L}^{\infty}(\mathcal{T})=\prod_{j=1}^{N} L^{\infty}\left(0, \ell_{j}\right) .
$$

The space $\mathbb{L}^{2}(\mathcal{T})$ is equipped with the inner product

$$
(\underline{u}, \underline{v})_{\mathbb{L}^{2}(\mathcal{T})}=\sum_{j=1}^{N} \int_{0}^{\ell_{j}} u_{j} v_{j} d x, \quad \forall \underline{u}, \underline{v} \in \mathbb{L}^{2}(\mathcal{T}) .
$$

We also define the space

$$
\mathbb{B}=C\left([0, T], \mathbb{L}^{2}(\mathcal{T})\right) \cap L^{2}\left(0, T ; \mathbb{H}_{e}^{1}(\mathcal{T})\right)
$$

endowed with the norm

$$
\|u\|_{\mathbb{B}}=\|\underline{u}\|_{C\left([0, T], \mathbb{L}^{2}(\mathcal{T})\right)}+\|\underline{u}\|_{L^{2}\left(0, T ; \mathbb{H}_{e}^{1}(\mathcal{T})\right)}=\max _{t \in[0, T]}\|\underline{u}\|_{\mathbb{L}^{2}(\mathcal{T})}+\left(\int_{0}^{T}\|\underline{u}(t, \cdot)\|_{\mathbb{H}_{e}^{1}(\mathcal{T})}^{2} d t\right)^{1 / 2} .
$$

Note first (1.1) and (1.2) imply

$$
\omega_{j}=\operatorname{supp} b_{j} \subset \operatorname{supp} a_{j} \text {, and } a_{j}(x) \geq b_{0}+c_{0}>0 \text {, in } \omega_{j} .
$$

To deal with delays we introduce the following space

$$
\mathcal{H}=\mathbb{L}^{2}(\mathcal{T}) \times\left(\prod_{j=1}^{N} L^{2}\left(\left(-h_{j}, 0\right) \times\left(0, \ell_{j}\right)\right)\right)
$$

endowed with

$$
\|(\underline{u}, \underline{z})\|_{\mathcal{H}}^{2}=\sum_{j=1}^{N}\left(\int_{0}^{\ell_{j}} u_{j}(x)^{2} d x+\int_{-h_{j}}^{0} \int_{0}^{\ell_{j}} \xi_{j}(x) z_{j}^{2}(s, x) d x d s\right)
$$

where for all $j=1, \cdots, N, \xi_{j}$ is a non-negative function belonging to $L^{\infty}\left(0, \ell_{j}\right)$ such that $\operatorname{supp} \xi_{j}=\operatorname{supp} b_{j}=\omega_{j}$ and

$$
b_{j}(x)+c_{0} \leq \xi_{j}(x) \leq 2 a_{j}(x)-b_{j}(x)-c_{0}, \text { in } \omega_{j} .
$$

For $(\mathrm{KdVd})$, we define the energy

$$
E(t)=\sum_{j=1}^{N}\left(\int_{0}^{\ell_{j}} u_{j}^{2}(t, x) d x+h_{j} \int_{\omega_{j}} \int_{0}^{1} \xi_{j}(x) u_{j}^{2}\left(t-h_{j} \rho, x\right) d \rho d x\right) .
$$

The above expression corresponds to the square norm of $(\underline{u}(t, \cdot), \underline{u}(t+\cdot, \cdot))$ in $\mathcal{H}$, with the change of variable $s=-h_{j} \rho$ for $u_{j}(t+s, x)$. 
Finally we denote $\mathbb{L}^{2}(\Omega)=\prod_{j=1}^{N} L^{2}\left((0,1) \times \omega_{j}\right)$, and let

$$
H=\mathbb{L}^{2}(\mathcal{T}) \times\left(\prod_{j=1}^{N} L^{2}\left((0,1) \times \omega_{j}\right)\right)=\mathbb{L}^{2}(\mathcal{T}) \times \mathbb{L}^{2}(\Omega)
$$

with its inner product

$$
\left\langle(\underline{\underline{u}}),\left(\frac{\underline{v}}{\underline{y}}\right)\right\rangle=\sum_{j=1}^{N} \int_{0}^{\ell_{j}} u_{j}(x) v_{j}(x) d x+h_{j} \int_{\omega_{j}} \int_{0}^{1} \xi_{j}(x) z_{j}(\rho, x) y_{j}(\rho, x) d \rho d x,
$$

we denote by $\|\cdot\|_{H}$ its associated norm.

Our first main result is the following one, where local exponential stability of $\mathrm{KdVd}$ is obtained for a restricted assumption over $L=\max _{j=1 \ldots, N} \ell_{j}$, but an estimation of the decay rate is given.

Theorem 1.1 Assume $\underline{a}, \underline{b} \in \mathbb{L}^{\infty}(\mathcal{T})$ componentwise non-negative that satisfy 1.1 and (1.2). Let $\alpha>\frac{N}{2}$ and $\left(\ell_{j}\right)_{j=1}^{N} \subset(0, \infty)$ such that $L<\frac{\sqrt{3}}{2} \pi$. Then there exists $\epsilon>0$, such that for every $\left(\underline{u}^{0}, \underline{z}^{0}(-\underline{h} \cdot, \cdot)\right) \in H$ satisfying $\left\|\left(\underline{u}^{0}, \underline{z}^{0}(-\underline{h} \cdot, \cdot)\right)\right\|_{H} \leq \epsilon$, the energy of (KdVd) defined by (1.6) decays exponentially. That is, there exist $C>0, \gamma>0$ such that

$$
E(t) \leq C E(0) e^{-2 \gamma t}, \quad t>0,
$$

where

$$
\begin{gathered}
\gamma \leq \min \left\{\frac{\left(3 \mu_{1} \pi^{2}+\frac{2}{3} L^{3 / 2} \epsilon \mu_{1} \pi^{2}-\mu_{1} 4 L^{2}\right)}{8 L^{2}\left(\left(1+L \mu_{1}\right)\right)} \min _{j=1, \cdots, N} \frac{\mu_{2}}{2 h_{j}\left(\mu_{2}+\left\|\xi_{j}\right\|_{L^{\infty}\left(0, \ell_{j}\right)}\right)}\right\}, \\
C=\left(1+\max \left\{L \mu_{1}, \frac{\mu_{2}}{b_{0}}\right\}\right),
\end{gathered}
$$

for $\mu_{1}$ and $\mu_{2}$ such that

$$
\begin{aligned}
0<\mu_{1}<\min & \left\{1, \frac{1}{N}(2 \alpha-N) \min _{j=1, \cdots, N}\left\{\inf _{\omega_{j}} \frac{2 a_{j}-b_{j}-\xi_{j}}{L b_{j}}, \inf _{\omega_{j}} \frac{\xi_{j}-b_{j}}{L b_{j}}\right\}\right\}, \\
0 & <\mu_{2}<\min _{j=1, \cdots, N} \inf _{\omega_{j}}\left\{2 a_{j}-b_{j}-\xi_{j}-\mu_{1} L b_{j}\right\} .
\end{aligned}
$$

This result will be proved in a constructive way by using a Lyapunov function, similar to those used in [2, 21].

On the other hand, our second main result is obtained without restriction on the lengths of $\mathcal{T}$ and gives us a local exponential stability. 
Theorem 1.2 Assume $\underline{a}, \underline{b} \in \mathbb{L}^{\infty}(\mathcal{T})$ componentwise non-negative that satisfy 1.1 and 1.2 . Let $\left(\ell_{j}\right)_{j=1}^{N} \subset(0, \infty)$, then there exists $\epsilon>0$ such that for all $\left(\underline{u}^{0}, \underline{z}^{0}(-\underline{h} \cdot, \cdot)\right) \in$ $H$ with $\left\|\left(\underline{u}^{0}, \underline{z}^{0}(-\underline{h} \cdot, \cdot)\right)\right\|_{H} \leq \epsilon$ the energy of $\mathrm{KdVd}$ decays exponentially, i.e, there exists $C>0$ and $\mu>0$ such that $E(t) \leq C E(0) e^{-\mu t}$ for all $t>0$.

The main difference between Theorem 1.1 and Theorem 1.2 is that Theorem 1.2 is based on an observability inequality which is proved using a contradiction argument. Thus we can not estimate the decay rate.

In our third main result, we proved a semi-global exponential stabilization by working directly with the nonlinear system (KdVd).

Theorem 1.3 Assume $\underline{a}, \underline{b} \in \mathbb{L}^{\infty}(\mathcal{T})$ componentwise non-negative that satisfies 1.1 and (1.2). Let $\left(\ell_{j}\right)_{j=1}^{N} \subset(0, \infty)$ and $R>0$. Then for all $\left(\underline{u}^{0}, \underline{z}^{0}(-\underline{h} \cdot, \cdot)\right) \in H$ with $\left\|\left(\underline{u}^{0}, \underline{z}^{0}(-\underline{h} \cdot, \cdot)\right)\right\|_{H} \leq R$ then there exist $C=C(R)>0$ and $\mu=\mu(R)>0$ such that the

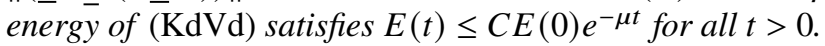

The semi-global sense of this result arises from the fact that we can choose as we want the parameter $R>0$ of the initial data but the decay rate depends on it.

In the last results presented, it is not possible to take $a_{j}=0$ and $b_{j} \neq 0$ for some $j \in\{1, \cdots, N\}$ (by (1.4) if $a_{j}=0$ then $b_{j}=0$ ). However this is only a technical part of the proof and in the next result we deal with this problem in a more general case, we suppose for this part that

$$
\omega_{j}=\operatorname{supp} b_{j} \not \subset \operatorname{supp} a_{j}, \text { for } j \in I \subset\{1, \cdots, N\} .
$$

For this we write now the analogues of the condition $(1.2)$ in the setting $(1.8)$, take $I^{*}=\{1, \cdots, N\} \backslash I$,

there exists $c_{0}>0$, such that $b_{j}(x)+c_{0} \leq a_{j}(x), \forall x \in \omega_{j}$, for $j \in I^{*}$.

Then we write our last result of stabilization when the internal delay is not necessarily supported in the domain of $a_{j}$.

Theorem 1.4 Assume $\underline{a}, \underline{b} \in \mathbb{L}^{\infty}(\mathcal{T})$ componentwise non-negative that satisfy 1.1 and 1.9. Let $\alpha>\frac{N}{2}, \eta>1$ and $\left(\ell_{j}\right)_{j=1}^{N} \subset(0,+\infty)$ such that $L<\frac{\sqrt{3}}{2} \pi$. Then there exits $\delta=\delta(\alpha, \eta, L, \underline{h})>0$ and $\epsilon>0$, such that for every $\left(\underline{u}^{0}, \underline{z}^{0}(-\underline{h} \cdot, \cdot)\right.$ satisfying $\|\underline{b}\|_{\mathbb{L}^{\infty}(\mathcal{T})} \leq \delta$ and $\left\|\left(\underline{u}^{0}, \underline{z}^{0}(-\underline{h} \cdot, \cdot)\right)\right\|_{H} \leq \epsilon$, the energy of $(\mathrm{KdVd})$ decays exponentially to 0 .

The organization of this paper is the following:

Section 2 is devoted to the study of the well-posedness of ( $\mathrm{KdVd}$. More precisely we consider the linearization around 0 of $(\mathrm{KdVd})$ and using Semigroup Theory we show the well-posedness of the linear system. Then using a fixed point argument we obtain the well-posedness for the nonlinear system. In Section 3 we present our stabilization results when the feedback terms $\underline{a}, \underline{b} \in \mathbb{L}^{\infty}(\mathcal{T})$ satisfy $(1.1)$ and $(1.2)$. The first one namely Theorem 1.1 is obtained following the same steps as [2, 21]. Then we 
detail the proofs of Theorem 1.2 and Theorem 1.3 that are based on an observability inequality. In Section 4 , we study the case where (1.2) is not satisfied and we show the proof of Theorem 1.4 using a suitable auxiliary system and a perturbation argument. Some numerical simulations are presented in Section 5 in order to illustrate the results obtained. Section 6 collects some concluding ideas and future research lines.

\section{Well-posedness of a delayed KdV system}

Our idea is the following, first we work with the linearization around 0 of $(\mathrm{KdVd})$, then we add a boundary source term at the central node $g(t)$ to consider the nonlinear boundary condition $-\frac{N}{3} u_{1}^{2}(t, 0)$ and secondly we add the internal source terms $f_{j}$ to consider after the term $u_{j} \partial_{x} u_{j}$. Finally to pass to the nonlinear $\mathrm{KdVd}$ we use a fixed point argument.

\subsection{Well-posedness of the linear case}

We start by proving the well-posedness for the linearization of $(\overline{\mathrm{KdVd}})$ around 0 , that writes

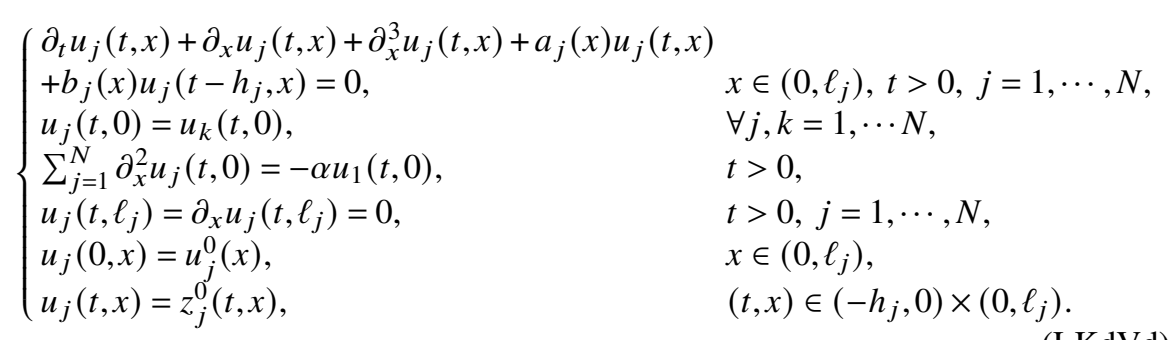

$(\mathrm{LKdVd})$

We set $z_{j}(t, \rho, x)=\left.u_{j}\right|_{\omega_{j}}\left(t-h_{j} \rho, x\right) x \in \omega_{j}, \rho \in(0,1)$. Then we can check that

$$
\begin{cases}h_{j} \partial_{t} z_{j}(t, \rho, x)+\partial_{\rho} z_{j}(t, \rho, x)=0, & x \in \omega_{j}, \rho \in(0,1), t>0, \\ z_{j}(t, 0, x)=u_{j}(t, x), & x \in \omega_{j}, t>0, \\ z_{j}(0, \rho, x)=\left.u_{j}\right|_{\omega_{j}}\left(-h_{j} \rho, x\right)=z_{j}^{0}\left(-h_{j} \rho, x\right), \rho \in(0,1) .\end{cases}
$$

Let us introduce the componentwise product $*$ as

$$
\left(\begin{array}{c}
p_{1} \\
\vdots \\
p_{N}
\end{array}\right) \cdot *\left(\begin{array}{c}
q_{1} \\
\vdots \\
q_{N}
\end{array}\right)=\left(\begin{array}{c}
p_{1} q_{1} \\
\vdots \\
p_{N} q_{N}
\end{array}\right) .
$$

Then (LKdVd) can be written as

$$
\left\{\begin{array}{c}
U_{t}(t)=\mathcal{A} U(t), t>0 \\
U(0)=U_{0}
\end{array}\right.
$$


where $U=(\underline{u}), U_{0}=\left(\left.\underline{z}^{0}\right|_{\omega} \underline{u}^{0}(-\underline{h} \cdot, \cdot)\right)$ and the operator $\mathcal{A}$ is defined by:

$$
\mathcal{A} U=\left(\begin{array}{c}
-\left(\mathrm{D}_{x}(\mathcal{T})+\mathrm{D}_{x}^{3}(\mathcal{T})\right) \underline{u}-\underline{a} \cdot * \underline{u}-\underline{b} \cdot * \underline{\tilde{z}}(1, \cdot) \\
-\frac{1}{\underline{h}} \cdot * \mathrm{D}_{\rho}(\mathcal{T}) \underline{z}
\end{array}\right)
$$

for $\underline{u}=\left(u_{j}\right)_{j=1}^{N}, \underline{a}=\left(a_{j}\right)_{j=1}^{N}, \underline{b}=\left(b_{j}\right)_{j=1}^{N}, \underline{h}=\left(h_{j}\right)_{j=1}^{N},\left(\frac{1}{\underline{h}}\right)_{j}=\frac{1}{h_{j}}$ and $\underline{z}(1, \cdot)=$ $\left(\tilde{z}_{j}(1, \cdot)\right)_{j=1}^{N}$ in which $\tilde{z}_{j}(1, \cdot) \in L^{2}\left(0, \ell_{j}\right)$ is the extension by 0 of $z_{j}(1, \cdot)$ outside $\omega_{j}$ and the operators $\mathrm{D}_{x}(\mathcal{T})\left(\right.$ resp. $\mathrm{D}_{\rho}(\mathcal{T})$ ) acts like the derivative with respect to $x$ (resp $\rho$ ) componentwise as

$$
\mathrm{D}_{x}(\mathcal{T})\left(\begin{array}{c}
u_{1} \\
\vdots \\
u_{N}
\end{array}\right)=\left(\begin{array}{c}
\partial_{x} u_{1} \\
\vdots \\
\partial_{x} u_{N}
\end{array}\right), \quad \mathrm{D}_{\rho}(\mathcal{T})\left(\begin{array}{c}
z_{1} \\
\vdots \\
z_{N}
\end{array}\right)=\left(\begin{array}{c}
\partial_{\rho} z_{1} \\
\vdots \\
\partial_{\rho} z_{N}
\end{array}\right)
$$

The domain of $\mathcal{A}$ is the following

$$
\begin{gathered}
D(\mathcal{A})=\left\{\left(\frac{\underline{u}}{\underline{z}}\right), \underline{u} \in\left(\prod_{j=1}^{N} H^{3}\left(0, \ell_{j}\right)\right) \cap \mathbb{H}_{e}^{2}(\mathcal{T}), \sum_{j=1}^{N} \frac{\mathrm{d}^{2} u_{j}}{\mathrm{~d} x^{2}}(0)=-\alpha u_{1}(0),\right. \\
\left.\underline{z} \in \prod_{j=1}^{N} L^{2}\left(H^{1}(0,1) \times \omega_{j}\right), z_{j}(0, x)=\left.u_{j}\right|_{\omega_{j}}(x)\right\} .
\end{gathered}
$$

Note that if $(\underline{\underline{u}}) \in D(\mathcal{A})$ then $\underline{u} \in \mathbb{H}_{e}^{2}(\mathcal{T})$ that implies $u_{j}\left(t, \ell_{j}\right)=\partial_{x} u_{j}\left(t, \ell_{j}\right)=0$.

Theorem 2.1 Assume $\underline{a}, \underline{b} \in \mathbb{L}^{\infty}(\mathcal{T})$ componentwise non-negative that satisfy 1.1 and (1.2). Let $U_{0} \in H$. Then there exists a unique solution $U \in C([0, \infty) ; H)$ of $(2.2)$. Moreover if $U_{0} \in D(\mathcal{A})$ then $U$ is a classical solution and

$$
U \in C([0, \infty) ; D(\mathcal{A})) \cap C^{1}([0, \infty) ; H) .
$$

Proof Let $U=\left(\frac{\underline{u}}{\underline{z}}\right) \in D(\mathcal{A})$, then 


$$
\begin{gathered}
\langle\mathcal{A} U, U\rangle=\sum_{j=1}^{N}\left(\int_{0}^{\ell_{j}}\left(-\partial_{x}^{3} u_{j}(x)-\partial_{x} u_{j}(x)-a_{j}(x) u_{j}(x)\right) u_{j}(x) d x\right. \\
\left.-\int_{\omega_{j}} b_{j}(x) z_{j}(1, x) u_{j}(x) d x-h_{j} \int_{\omega_{j}} \int_{0}^{1} \xi_{j}(x) \frac{1}{h_{j}} \partial_{\rho} z_{j}(\rho, x) z_{j}(\rho, x) d \rho d x\right) \\
=\sum_{j=1}^{N}\left(\int_{0}^{\ell_{j}} \partial_{x}^{2} u_{j}(x) \partial_{x} u_{j}(x) d x-\left.\partial_{x}^{2} u_{j}(x) u_{j}(x)\right|_{0} ^{\ell_{j}}-\left.\frac{1}{2} u_{j}^{2}(x)\right|_{0} ^{\ell_{j}}-\int_{0}^{\ell_{j}} a_{j}(x) u_{j}^{2}(x) d x\right. \\
\left.-\int_{\omega_{j}} b_{j}(x) z_{j}(1, x) u_{j}(x) d x-\left.\frac{1}{2} \int_{\omega_{j}} \xi_{j}(x) z_{j}^{2}(\rho, x)\right|_{0} ^{1} d x\right) \\
=\sum_{j=1}^{N}\left(\left.\frac{1}{2}\left(\partial_{x} u_{j}(x)\right)^{2}\right|_{0} ^{\ell_{j}}+\partial_{x}^{2} u_{j}(0) u_{1}(0)+\frac{1}{2} u_{1}^{2}(0)-\int_{0}^{\ell_{j}} a_{j}(x) u_{j}^{2}(x) d x\right. \\
\left.-\int_{\omega_{j}} b_{j}(x) z_{j}(1, x) u_{j}(x) d x-\frac{1}{2} \int_{\omega_{j}} \xi_{j}(x) z_{j}^{2}(1, x) d x+\frac{1}{2} \int_{\omega_{j}} \xi_{j}(x) z_{j}^{2}(0, x) d x\right) .
\end{gathered}
$$

Thus,

$$
\begin{gathered}
\langle\mathcal{A} U, U\rangle \leq-\frac{1}{2} \sum_{j=1}^{N}\left(\partial_{x} u_{j}(0)\right)^{2}+\left(\frac{N}{2}-\alpha\right) u_{1}^{2}(0)-\sum_{j=1}^{N} \int_{\left(0, \ell_{j}\right) / \omega_{j}} a_{j}(x) u_{j}^{2}(x) d x \\
+\sum_{j=1}^{N} \int_{\omega_{j}}\left(-a_{j}(x)+\frac{b_{j}(x)}{2}+\frac{\xi_{j}(x)}{2}\right) u_{j}^{2}(x) d x+\sum_{j=1}^{N} \int_{\omega_{j}}\left(\frac{b_{j}(x)}{2}-\frac{\xi_{j}(x)}{2}\right) z_{j}^{2}(1, x) d x .
\end{gathered}
$$

Using [1.4, , 1.5) and that $\alpha \geq \frac{N}{2}$ we conclude that $\langle\mathcal{A} U, U\rangle \leq 0$, thus $\mathcal{A}$ is dissipative. Easy calculations show that

$$
\mathcal{A}^{*}(\underline{\underline{y}})=\left(\begin{array}{c}
\left(\mathrm{D}_{x}(\mathcal{T})+\mathrm{D}_{x}^{3}(\mathcal{T})\right) \underline{v}-\underline{a} \cdot * \underline{v}+\underline{\xi} \cdot * \underline{\tilde{y}}(0, \cdot) \\
\frac{1}{\underline{h}} * * \mathrm{D}_{\rho}(\mathcal{T}) \underline{y}
\end{array}\right),
$$

in which $\tilde{y}_{j}(0, \cdot) \in L^{2}\left(0, \ell_{j}\right)$ is the extension by 0 of $y_{j}(0, \cdot)$ outside $\omega_{j}$ and with

$$
\begin{gathered}
D\left(\mathcal{A}^{*}\right)=\left\{(\underline{\underline{v}}), \underline{v} \in\left(\prod_{j=1}^{N} H^{3}\left(0, \ell_{j}\right)\right) \cap \mathbb{H}_{e}^{1}(\mathcal{T}), \sum_{j=1}^{N} \frac{\mathrm{d}^{2} v_{j}}{\mathrm{~d} x^{2}}(0)=(\alpha-N) v_{1}(0),\right. \\
\left.\partial_{x} v_{j}(0)=0, \forall j=1, \cdots, N, \underline{y} \in \prod_{j=1}^{N} L^{2}\left(H^{1}(0,1) \times \omega_{j}\right), y_{j}(1, x)=-\left.\frac{b_{j}(x)}{\xi_{j}(x)} v_{j}\right|_{\omega_{j}}(x)\right\} .
\end{gathered}
$$

Note that $\left(\frac{\underline{v}}{\underline{y}}\right) \in D\left(\mathcal{A}^{*}\right)$ then $\underline{v} \in \mathbb{H}_{e}^{1}(\mathcal{T})$ that implies $v_{j}\left(t, \ell_{j}\right)=0$. Let $V=\left(\begin{array}{l}\underline{v} \\ \underline{y}\end{array}\right) \in$ $D\left(\mathcal{A}^{*}\right)$, then 


$$
\begin{gathered}
\left\langle\mathcal{A}^{*} V, V\right\rangle=\sum_{j=1}^{N}\left(\int_{0}^{\ell_{j}}\left(\partial_{x}^{3} v_{j}(x)+\partial_{x} v_{j}(x)-a_{j}(x) v_{j}(x)\right) v_{j}(x) d x\right. \\
\left.+\int_{\omega_{j}} \xi_{j}(x) y_{j}(0, x) v_{j}(x) d x+\int_{\omega_{j}} h_{j} \int_{0}^{1} \xi_{j}(x) \frac{1}{h_{j}} \partial_{\rho} y_{j}(\rho, x) y_{j}(\rho, x) d \rho d x\right), \\
=\sum_{j=1}^{N}\left(-\int_{0}^{\ell_{j}} \partial_{x}^{2} v_{j}(x) \partial_{x} v_{j}(x) d x+\left.\partial_{x}^{2} v_{j}(x) v_{j}(x)\right|_{0} ^{\ell_{j}}+\left.\frac{1}{2}\left|v_{j}(x)\right|^{2}\right|_{0} ^{\ell_{j}}\right. \\
\left.-\int_{0}^{\ell_{j}} a_{j}(x) v_{j}^{2}(x) d x+\int_{\omega_{j}} \xi_{j}(x) y_{j}(0, x) v_{j}(x) d x+\left.\frac{1}{2} \int_{\omega_{j}} \xi_{j}(x)\left|y_{j}(\rho, x)\right|^{2}\right|_{0} ^{1} d x\right) \\
\leq \sum_{j=1}^{N}\left(-\left.\frac{1}{2}\left|\partial_{x} v_{j}(x)\right|^{2}\right|_{0} ^{\ell_{j}}-\partial_{x}^{2} v_{j}(0) v_{1}(0)-\frac{1}{2} v_{1}^{2}(0)-\int_{0}^{\ell_{j}} a_{j}(x) v_{j}^{2}(x) d x\right. \\
\left.\leq \int_{\omega_{j}} \xi_{j}(x) y_{j}^{2}(0, x) d x+\frac{1}{2} \int_{\omega_{j}} \xi_{j}(x) v_{j}^{2}(x) d x+\frac{1}{2} \int_{\omega_{j}} \xi_{j}(x)\left(y_{j}^{2}(1, x)-y_{j}^{2}(0, x)\right) d x\right), \\
\leq-\frac{1}{2} \sum_{j=1}^{N}\left|\partial_{x} v_{j}\left(\ell_{j}\right)\right|^{2}+\left(\frac{N}{2}-\alpha\right) v_{1}^{2}(0)+\int_{\omega_{j}}\left(-a_{j}(x)+\frac{\xi_{j}(x)}{2}+\frac{b_{j}^{2}(x)}{2 \xi_{j}(x)}\right) v_{j}^{2}(x) d x \\
-\int_{\left(0, \ell_{j}\right) / \omega_{j}} a_{j}(x) v_{j}^{2}(x) d x-\frac{1}{2} \int_{\omega_{j}} \xi_{j}(x) y_{j}^{2}(0, x) d x .
\end{gathered}
$$

Moreover we know that $\xi_{j}(x)>b_{j}(x)>b_{0}>0$, for $x \in \omega_{j}$, then we have that $\frac{b_{j}^{2}(x)}{\xi_{j}(x)}<$ $b_{j}(x)$, for $x \in \omega_{j}$ and then

$$
-a_{j}(x)+\frac{\xi_{j}(x)}{2}+\frac{b_{j}^{2}(x)}{2 \xi_{j}(x)}<-a_{j}(x)+\frac{\xi_{j}(x)}{2}+\frac{b_{j}(x)}{2} \leq 0, \quad \text { for } x \in \omega_{j},
$$

thus as $\alpha \geq \frac{N}{2}, \mathcal{A}^{*}$ is dissipative. Finally $\mathcal{A}$ and $\mathcal{A}^{*}$ are dissipative, also $\mathcal{A}$ is densely defined closed operator, thus $\mathcal{A}$ is the infinitesimal generator of a $C_{0}$ semigroup of contractions on $H$ [16].

As the systems ( $(\mathrm{LKdVd})$ and $(2.2)$ are equivalent we obtain the well-posedness of (LKdVd). Let $S(t), t \geq 0$ the semigroup of contractions associated with $\mathcal{A}$. Next result gives us some a priori estimates for $[\mathrm{LKdVd}$.

Proposition 2.1 Assume $\underline{a}, \underline{b} \in \mathbb{L}^{\infty}(\mathcal{T})$ componentwise non-negative that satisfy 1.1 , and (1.2). Then, the map

$$
U_{0}=\left(\underline{u}^{0}, \underline{z}^{0}(-\underline{h} \cdot, \cdot)\right) \mapsto S(\cdot)\left(\underline{u}^{0}, \underline{z}^{0}(-\underline{h} \cdot, \cdot)\right)
$$


is continuous from $H$ to $\mathbb{B} \times C\left([0, T] ; \mathbb{L}^{2}(\Omega)\right)$ and for $\left(\underline{u}^{0}, \underline{z}^{0}(-\underline{h} \cdot, \cdot)\right) \in H$ the following estimates hold

$$
\begin{gathered}
\sum_{j=1}^{N} \int_{0}^{T} \int_{0}^{\ell_{j}} a_{j}(x)\left(u_{j}(t, x)\right)^{2} d x d t+\int_{0}^{T} \int_{\omega_{j}}\left(z_{j}(t, 1, x)\right)^{2} d x d t \\
\leq C\left(\left\|\underline{u}^{0}\right\|_{\mathbb{L}^{2}(\mathcal{T})}^{2}+\left\|\underline{z}^{0}(-\underline{h} \cdot, \cdot)\right\|_{\mathbb{L}^{2}(\Omega)}^{2}\right), \\
+\underline{\|} \underline{u}^{0}\left\|_{\mathbb{L}^{2}(\mathcal{T})}^{2} \leq\left(\frac{1+2 T\|\underline{a}\|_{\mathbb{L}^{\infty}(\mathcal{T})}+2 T\|\underline{b}\|_{\mathbb{L}^{\infty}(\mathcal{T})}}{T}\right)\right\| \underline{u} \|_{L^{2}\left(0, T ; \mathbb{L}^{2}(\mathcal{T})\right)}^{2} \\
+2\left(\alpha-\frac{N}{2}\right)\left\|u_{1}(\cdot, 0)\right\|_{L^{2}(0, T)}^{2}+\left\|\partial_{x} \underline{u}(\cdot, 0)\right\|_{L^{2}(0, T)}^{2}+\|\underline{b}\|_{\mathbb{L}^{\infty}(\mathcal{T})}\left\|\underline{z}^{0}(-\underline{h} \cdot, \cdot)\right\|_{\mathbb{L}^{2}(\Omega)}^{2}, \\
\left\|\underline{z}^{0}(-\underline{h}, \cdot)\right\|_{\mathbb{L}^{2}(\Omega)}^{2} \leq\|\underline{z}(T, \cdot, \cdot)\|_{\mathbb{L}^{2}(\Omega)}^{2}+\sum_{j=1}^{N} \frac{1}{h_{j}} \int_{0}^{T} \int_{\omega_{j}}\left|z_{j}(t, 1, x)\right|^{2} d x d t .
\end{gathered}
$$

Proof Taking $\left(\underline{u}^{0}, \underline{z}^{0}(-\underline{h} \cdot, \cdot)\right) \in H$, Theorem 2.1 gives us $S(\cdot)\left(\underline{u}^{0}, \underline{z}^{0}(-\underline{h} \cdot, \cdot)\right)=(\underline{u}, \underline{z}) \in$ $C([0, T] ; H)$ and using that $\mathcal{A}$ generates a $C_{0}$ semigroup of contractions, we get all $t \in[0, T]$

$$
\begin{aligned}
& \sum_{j=1}^{N} \int_{0}^{\ell_{j}}\left(u_{j}(t, x)\right)^{2} d x+\sum_{j=1}^{N} h_{j} \int_{\omega_{j}} \int_{0}^{1} \xi_{j}(x)\left(z_{j}(t, \rho, x)\right)^{2} d \rho d x \\
\leq & \sum_{j=1}^{N} \int_{0}^{\ell_{j}}\left(u_{j}^{0}(x)\right)^{2} d x+\sum_{j=1}^{N} h_{j} \int_{\omega_{j}} \int_{0}^{1} \xi_{j}(x)\left(z_{j}^{0}\left(-h_{j} \rho, x\right)\right)^{2} d \rho d x .
\end{aligned}
$$

Let $\underline{p} \in \prod_{j=1}^{N} C^{\infty}([0, T] \times(0,1))$ and $\underline{q} \in \prod_{j=1}^{N} C^{\infty}\left([0, T] \times\left(0, \ell_{j}\right)\right)$. Now multiplying

LKdVd by $q_{j} u_{j}$ and 2.1 by $p_{j} z_{j}$ and integrating on $(0, s) \times\left(0, \ell_{j}\right)$ and $(0, s) \times$ $(0,1) \times \omega_{j}$ we can obtain

$$
\begin{gathered}
\left.\int_{0}^{\ell_{j}} q_{j}(t, x)\left|u_{j}(t, x)\right|^{2} d x\right|_{0} ^{s}-\int_{0}^{s} \int_{0}^{\ell_{j}}\left(\partial_{t} q_{j}+\partial_{x} q_{j}+\partial_{x}^{3} q_{j}\right)\left|u_{j}\right|^{2} d x d t \\
+2 \int_{0}^{s} \int_{0}^{\ell_{j}} a_{j} q_{j}\left|u_{j}\right|^{2} d x d t+2 \int_{0}^{s} \int_{0}^{\ell_{j}} b_{j}(x) q_{j}(t, x) u_{j}\left(t-h_{j}, x\right) u_{j}(t, x) d x d t \\
+3 \int_{0}^{s} \int_{0}^{\ell_{j}}\left|\partial_{x} u_{j}\right|^{2} \partial_{x} q_{j} d x d t=\int_{0}^{s}\left[\left(q_{j}+\partial_{x}^{2} q_{j}\right)\left|u_{j}\right|^{2}+2 q_{j} u_{j} \partial_{x}^{2} u_{j}\right. \\
\left.-2 \partial_{x} q_{j} u_{j} \partial_{x} u_{j}-q_{j}\left|\partial_{x} u_{j}\right|^{2}\right](t, 0) d t, \\
\left.\int_{0}^{1} \int_{\omega_{j}}\left(z_{j}(t, \rho, x)\right)^{2} p_{j}(t, \rho) d x d \rho\right|_{0} ^{s}-\frac{1}{h_{j}} \int_{0}^{s} \int_{0}^{1} \int_{\omega_{j}}\left(h_{j} \partial_{t} p_{j}+\partial_{\rho} p_{j}\right)\left|z_{j}\right|^{2} d x d \rho d t \\
+\frac{1}{h_{j}} \int_{0}^{s} \int_{\omega_{j}}\left(z_{j}(t, 1, x)\right)^{2} p_{j}(t, 1)-\left(u_{j}(t, x)\right)^{2} p_{j}(t, 0) d x d t=0 .
\end{gathered}
$$


Taking $s=T$ and $p_{j}=\rho$ in 2.10 we get

$$
\begin{gathered}
\int_{0}^{1} \int_{\omega_{j}} \rho\left[z_{j}(T, \rho, x)^{2}-z_{j}^{0}\left(-\rho h_{j}, x\right)^{2}\right] d x d \rho-\frac{1}{h_{j}} \int_{0}^{T} \int_{0}^{1} \int_{\omega_{j}}\left|z_{j}\right|^{2} d x d \rho d t \\
+\frac{1}{h_{j}} \int_{0}^{T} \int_{\omega_{j}} z_{j}(t, 1, x)^{2} d x d \rho=0 .
\end{gathered}
$$

Thus,

$$
\begin{gathered}
\frac{1}{h_{j}} \int_{0}^{T} \int_{\omega_{j}} z_{j}(t, 1, x)^{2} d x d \rho \\
\leq \frac{1}{h_{j}} \int_{0}^{T} \int_{0}^{1} \int_{\omega_{j}}\left|z_{j}\right|^{2} d x d \rho d t+\int_{0}^{1} \int_{\omega_{j}} \rho z_{j}^{0}\left(-\rho h_{j}, x\right)^{2} d x d \rho .
\end{gathered}
$$

and hence with 2.8 we get

$$
\sum_{j=1}^{N} \int_{\omega_{j}} z_{j}(t, 1, x)^{2} d x d \rho \leq C\left(\left\|\underline{u}^{0}\right\|_{\mathbb{L}^{2}(\mathcal{T})}^{2}+\left\|\underline{z}^{0}(-\underline{h} \cdot, \cdot)\right\|_{\mathbb{L}^{2}(\Omega)}^{2}\right) .
$$

Then taking $q_{j}=1$ in 2.9

$$
\begin{gathered}
\sum_{j=1}^{N} \int_{0}^{\ell_{j}}\left|u_{j}(s, x)\right|^{2} d x+\int_{0}^{s} \sum_{j=1}^{N}\left|\partial_{x} u_{j}(t, 0)\right|^{2} d t+(2 \alpha-N) \int_{0}^{s}\left|u_{1}(t, 0)\right|^{2} d t \\
+2 \sum_{j=1}^{N} \int_{0}^{s} \int_{0}^{\ell_{j}} a_{j}\left|u_{j}\right|^{2} d x d t+\sum_{j=1}^{N} 2 \int_{0}^{s} \int_{0}^{\ell_{j}} b_{j} u_{j}\left(t-h_{j}, x\right) u_{j}(t, x) d x d t \\
=\sum_{j=1}^{N} \int_{0}^{\ell_{j}}\left|u_{j}(0, x)\right|^{2} d x .
\end{gathered}
$$

Thus

$$
\begin{aligned}
& \sum_{j=1}^{N} \int_{0}^{\ell_{j}}\left|u_{j}(s, x)\right|^{2} d x+\int_{0}^{s} \sum_{j=1}^{N}\left|\partial_{x} u_{j}(t, 0)\right|^{2} d t+\sum_{j=1}^{N} 2 \int_{0}^{T} \int_{0}^{\ell_{j}} a_{j}\left|u_{j}\right|^{2} d x d t \\
& \quad \leq \sum_{j=1}^{N} \int_{0}^{\ell_{j}}\left|u_{j}(0, x)\right|^{2} d x+\sum_{j=1}^{N} 2 \int_{0}^{T} \int_{0}^{\ell_{j}} b_{j}\left|u_{j}\left(t-h_{j}, x\right) \| u_{j}(t, x)\right| d x d t .
\end{aligned}
$$

Note now that

$$
\begin{gathered}
2 \int_{0}^{s} \int_{0}^{\ell_{j}} b_{j}(x)\left|u_{j}\left(t-h_{j}, x\right)\right|\left|u_{j}(t, x)\right| d x d t \\
\leq \int_{0}^{s} \int_{0}^{\ell_{j}} b_{j}(x)\left|u_{j}\left(t-h_{j}, x\right)\right|^{2} d x d t+\int_{0}^{s} \int_{0}^{\ell_{j}} b_{j}(x)\left|u_{j}(t, x)\right|^{2} d x d t, \\
=\int_{0}^{s} \int_{0}^{\ell_{j}} b_{j}(x)\left|u_{j}(t, x)\right|^{2} d x d t+\int_{-h_{j}}^{s-h_{j}} \int_{\omega_{j}} b_{j}(x)\left|u_{j}(t, x)\right|^{2} d x d t, \\
\leq 2 \int_{0}^{s} \int_{0}^{\ell_{j}} b_{j}(x)\left|u_{j}(t, x)\right|^{2} d x d t+\int_{-h_{j}}^{0} \int_{\omega_{j}} b_{j}(x)\left|z_{j}^{0}(t, x)\right|^{2} d x d t,
\end{gathered}
$$


which implies

$2 \sum_{j=1}^{N} \int_{0}^{s} \int_{0}^{\ell_{j}} b_{j}(x)\left|u_{j}\left(t-h_{j}, x\right) \| u_{j}(t, x)\right| d x d t \leq C\left(\left\|\underline{u}^{0}\right\|_{\mathbb{L}^{2}(\mathcal{T})}^{2}+\left\|\underline{z}^{0}(-\underline{h} \cdot, \cdot)\right\|_{\mathbb{L}^{2}(\Omega)}^{2}\right)$.

Thus, we have

$$
\begin{aligned}
\sum_{j=1}^{N} \int_{0}^{\ell_{j}}\left|u_{j}(s, x)\right|^{2} d x & +\int_{0}^{T} \sum_{j=1}^{N}\left|\partial_{x} u_{j}(t, 0)\right|^{2} d t+\sum_{j=1}^{N} \int_{0}^{T} \int_{0}^{\ell_{j}} a_{j}(x)\left(u_{j}(t, x)\right)^{2} d x d t \\
& \leq C\left(\left\|\underline{u}^{0}\right\|_{\mathbb{L}^{2}(\mathcal{T})}^{2}+\left\|\underline{z}^{0}(-\underline{h} \cdot, \cdot)\right\|_{\mathbb{L}^{2}(\Omega)}^{2}\right)
\end{aligned}
$$

that brings 2.5 using 2.12).

Note also that $\sum_{j=1}^{N} \partial_{x} u_{j}(\cdot, 0) \in L^{2}(0, T)$. Moreover integrating 2.14 with respect to $s$ over $[0, T]$ we can obtain.

$$
\|\underline{u}\|_{L^{2}\left(0, T ; \mathbb{L}^{2}(\mathcal{T})\right)}^{2} \leq C T\left(\left\|\underline{u}^{0}\right\|_{\mathbb{L}^{2}(\mathcal{T})}^{2}+\left\|\underline{z}^{0}(-\underline{h} \cdot, \cdot)\right\|_{\mathbb{L}^{2}(\Omega)}^{2}\right) .
$$

We are going to consider the following multiplier presented in [4], $q_{j}(t, x)=\frac{x\left(2 \ell_{j}-x\right)}{\ell_{j}^{2}}$, this multiplier satisfies the next properties

$-q_{j}(t, 0)=0, \forall t \in[0, T]$.

$-0 \leq q_{j}(t, x) \leq 1, \forall(t, x) \in[0, T] \times\left[0, \ell_{j}\right]$.

$-0 \leq \partial_{x} q_{j}(t, x) \leq \frac{2}{\ell_{j}}, \forall(t, x) \in[0, T] \times\left[0, \ell_{j}\right]$.

- $\partial_{x}^{2} q_{j}(t, x)=-\frac{2}{\ell_{j}^{2}}, \forall(t, x) \in[0, T] \times\left[0, \ell_{j}\right]$.

Taking $q_{j}(t, x)=\frac{x\left(2 \ell_{j}-x\right)}{\ell_{j}^{2}}$ and $s=T$ in 2.9 we get

$$
\begin{gathered}
\sum_{j=1}^{N} \int_{0} \ell_{j} q_{j}(t, x)\left|u_{j}(T, x)\right|^{2} d x+2 \sum_{j=1}^{N} \int_{0}^{T} \int_{\omega_{j}} q_{j}(t, x) b_{j}(x) u_{j}\left(t-h_{j}, x\right) u_{j}(t, x) d x d t \\
+2 \int_{0}^{T} u_{1}(t, 0) \sum_{j=1}^{N} \frac{2}{\ell_{j}} \partial_{x} u_{j}(t, 0) d t+2 \sum_{j=1}^{N} \int_{0}^{T} \int_{0}^{\ell_{j}} q_{j}(t, x) a_{j}(x)\left|u_{j}(t, x)\right|^{2} d x d t \\
-\sum_{j=1}^{N} \int_{0}^{T} \int_{0}^{\ell_{j}} \partial_{x} q_{j}(t, x)\left|u_{j}(t, x)\right|^{2} d x d t+3 \sum_{j=1}^{N} \int_{0}^{T} \int_{0}^{\ell_{j}}\left|\partial_{x} u_{j}(t, x)\right|^{2} \partial_{x} q_{j}(t, x) d x d t \\
=\sum_{j=1}^{N} \int_{0} \ell_{j} q_{j}(0, x)\left|u_{j}^{0}\right|^{2} d x-\left(\sum_{j=1}^{N} \frac{2}{\ell_{j}^{2}}\right) \int_{0}^{T}\left|u_{1}(t, 0)\right|^{2} d t .
\end{gathered}
$$

and then recalling that $L=\max _{j=1, \cdots, N} \ell_{j}$ and taking $\ell=\min _{j=1 \cdots, N} \ell_{j}$ 


$$
\begin{gathered}
\frac{2}{L^{2}}\left\|u_{1}(\cdot, 0)\right\|_{L^{2}(0, T)}^{2} \leq \frac{2}{\ell^{2}}\|\underline{u}\|_{L^{2}\left(0, T ; \mathbb{L}^{2}(\mathcal{T})\right)}^{2}-2 \int_{0}^{T} u_{1}(t, 0) \sum_{j=1}^{N} \partial_{x} u_{j}(t, 0) \frac{2}{\ell_{j}} d t \\
-2 \sum_{j=1}^{N} \int_{0}^{T} \int_{\omega_{j}} q_{j}(t, x) b_{j}(x) u_{j}\left(t-h_{j}, x\right) u_{j}(t, x) d x d t+\left\|\underline{u}^{0}\right\|_{\mathbb{L}^{2}(\mathcal{T})}^{2} .
\end{gathered}
$$

Using Young's inequality, (2.13) and (2.15) we get that $u_{1}(\cdot, 0) \in L^{2}(0, T)$ and

$$
\left\|u_{1}(\cdot, 0)\right\|_{L^{2}(0, T)}^{2} \leq C\left(\left\|\underline{u}^{0}\right\|_{\mathbb{L}^{2}(\mathcal{T})}^{2}+\left\|\underline{z}^{0}(-\underline{h \cdot}, \cdot)\right\|_{\mathbb{L}^{2}(\Omega)}^{2}\right) .
$$

Now, let us choose $q_{j}=x$ and $s=T$ in 2.9

$$
\begin{aligned}
& \left.\int_{0}^{\ell_{j}} x\left|u_{j}\right|^{2} d x\right|_{0} ^{T} d x-\int_{0}^{T} \int_{0}^{\ell_{j}}\left|u_{j}\right|^{2} d x d t+2 \int_{0}^{T} \int_{0}^{\ell_{j}} x b_{j}(x) u_{j}\left(t-h_{j}, x\right) u_{j}(t, x) d x d t \\
& +2 \int_{0}^{T} \int_{0}^{\ell_{j}} a_{j}(x) x\left|u_{j}\right|^{2} d x d t+3 \int_{0}^{T} \int_{0}^{\ell_{j}}\left|\partial_{x} u_{j}\right|^{2} d x d t=\int_{0}^{T}-2 u_{j}(t, 0) \partial_{x} u_{j}(t, 0) d t
\end{aligned}
$$

Then

$$
\begin{gathered}
3 \sum_{j=1}^{N} \int_{0}^{T} \int_{0}^{\ell_{j}}\left|\partial_{x} u_{j}\right|^{2} d x d t \leq\left(1+2 L\|\underline{b}\|_{\mathbb{L}^{\infty}(\mathcal{T})}\right) \sum_{j=1}^{N} \int_{0}^{T} \int_{0}^{\ell_{j}}\left|u_{j}\right|^{2} d x d t \\
+L\|\underline{b}\|_{\mathcal{L}^{\infty}(\mathcal{T})} \sum_{j=1}^{N} \int_{-h_{j}}^{0} \int_{\omega_{j}}\left|z_{j}^{0}(t, x)\right|^{2} d x d t+L \sum_{j=1}^{N} \int_{0}^{\ell_{j}}\left|u_{j}(0, x)\right|^{2} d x \\
-2 \sum_{j=1}^{N} \int_{0}^{T} u_{1}(t, 0) \partial_{x} u_{j}(t, 0) d t
\end{gathered}
$$

and hence

$$
3 \sum_{j=1}^{N} \int_{0}^{T} \int_{0}^{\ell_{j}}\left|\partial_{x} u_{j}\right|^{2} d x d t \leq C\left(\left\|\underline{u}^{0}\right\|_{\mathbb{L}^{2}(\mathcal{T})}^{2}+\left\|\underline{z}^{0}(-\underline{h}, \cdot)\right\|_{\mathbb{L}^{2}(\Omega)}^{2}\right),
$$

that brings with (2.8) the continuity of the map (2.4) from $H$ to $\mathbb{B} \times C\left([0, T]: \mathbb{L}^{2}(\Omega)\right)$. Now taking $q_{j}=T-t$ and $s=T$ in 2.9 , we obtain,

$$
\begin{gathered}
-\int_{0}^{\ell_{j}} T\left|u_{j}(0, x)\right|^{2} d x+\int_{0}^{T} \int_{0}^{\ell_{j}}\left|u_{j}\right|^{2} d x d t+2 \int_{0}^{T} \int_{0}^{\ell_{j}} a_{j}(x)(T-t)\left|u_{j}\right|^{2} d x d t \\
+2 \int_{0}^{T} \int_{0}^{\ell_{j}} b_{j}(x)(T-t) u_{j}\left(t-h_{j}, x\right) u_{j}(t, x) d x d t=\int_{0}^{T}\left[(T-t)\left|u_{j}(t, 0)\right|^{2}\right. \\
\left.+2(T-t) u_{j}(t, 0) \partial_{x}^{2} u_{j}(t, 0)-(T-t)\left|\partial_{x} u_{j}(t, 0)\right|^{2}\right] d t,
\end{gathered}
$$


then

$$
\begin{gathered}
T \sum_{j=1}^{N} \int_{0}^{\ell_{j}}\left|u_{j}(0, x)\right|^{2} d x=\sum_{j=1}^{N}\left(\int_{0}^{T} \int_{0}^{\ell_{j}}\left|u_{j}\right|^{2} d x d t++2 \int_{0}^{T} \int_{0}^{\ell_{j}}(T-t) a_{j}\left|u_{j}\right|^{2} d x d t\right. \\
\left.+2 \int_{0}^{T} \int_{0}^{\ell_{j}} b_{j}(x)(T-t) u_{j}\left(t-h_{j}, x\right) u_{j}(t, x) d x d t\right)+(2 \alpha-N) \int_{0}^{T}(T-t)\left|u_{1}(t, 0)\right|^{2} d t \\
+\sum_{j=1}^{N} \int_{0}^{T}(T-t)\left|\partial_{x} u_{j}(t, 0)\right|^{2} d t .
\end{gathered}
$$

Finally we get (2.6), that is

$$
\begin{gathered}
\left\|\underline{u}^{0}\right\|_{\mathbb{L}^{2}(\mathcal{T})}^{2} \leq\left(\frac{1+2 T\|\underline{a}\|_{\mathbb{L}^{\infty}(\mathcal{T})}+2 T\|\underline{b}\|_{\mathbb{L}^{\infty}(\mathcal{T})}}{T}\right)\|\underline{u}\|_{L^{2}\left(0, T ; \mathbb{L}^{2}(\mathcal{T})\right)}^{2} \\
+2\left(\alpha-\frac{N}{2}\right)\left\|u_{1}(\cdot, 0)\right\|_{L^{2}(0, T)}^{2}+\left\|\partial_{x} \underline{u}(\cdot, 0)\right\|_{L^{2}(0, T)}^{2}+\|\underline{b}\|_{\mathbb{L}^{\infty}(\mathcal{T})}\left\|\underline{z}^{0}(-\underline{h} \cdot, \cdot)\right\|_{\mathbb{L}^{2}(\Omega)}^{2} .
\end{gathered}
$$

Lastly taking $p_{j}=1$ and $s=T$ in 2.10

$$
\begin{gathered}
\int_{0}^{1} \int_{\omega_{j}}\left|z_{j}(T, \rho, x)\right|^{2} d x d \rho-\int_{0}^{1} \int_{\omega_{j}}\left|z_{j}^{0}\left(-h_{j} \rho, x\right)\right|^{2} d x d \rho \\
+\frac{1}{h_{j}} \int_{0}^{T} \int_{\omega_{j}}\left[\left|z_{j}(t, 1, x)\right|^{2}-\left|u_{j}(t, x)\right|^{2}\right] d x d t=0
\end{gathered}
$$

and hence we obtain 2.7).

\subsection{Extra boundary conditions}

Following [1] we need now some regularity results for the linear delayed $\mathrm{KdV}$ equation with extra boundary source term $g(t)$ at the central node

$$
\begin{cases}\partial_{t} u_{j}(t, x)+\partial_{x} u_{j}(t, x)+\partial_{x}^{3} u_{j}(t, x)+a_{j}(x) u_{j}(t, x) & x \in\left(0, \ell_{j}\right), t>0, j=1, \cdots, N, \\ +b_{j}(x) u_{j}\left(t-h_{j}, x\right)=0, & \forall j, k=1, \cdots N, \\ u_{j}(t, 0)=u_{k}(t, 0), & t>0, \\ \sum_{j=1}^{N} \partial_{x}^{2} u_{j}(t, 0)=-\alpha u_{1}(t, 0)+g(t), & t>0, j=1, \cdots, N, \\ u_{j}\left(t, \ell_{j}\right)=\partial_{x} u_{j}\left(t, \ell_{j}\right)=0, & x \in\left(0, \ell_{j}\right), \\ u_{j}(0, x)=u_{j}^{0}(x), & (t, x) \in\left(-h_{j}, 0\right) \times\left(0, \ell_{j}\right) . \\ u_{j}(t, x)=z_{j}^{0}(t, x), & \end{cases}
$$

Recall that $z_{j}(t, \rho, x)=\left.u_{j}\right|_{\omega_{j}}\left(t-h_{j} \rho, x\right)$, for $x \in \omega_{j}, \rho \in(0,1)$ is solution of

$$
\begin{cases}h_{j} \partial_{t} z_{j}(t, \rho, x)+\partial_{\rho} z_{j}(t, \rho, x)=0, & x \in \omega_{j}, \rho \in(0,1), t>0, \\ z_{j}(t, 0, x)=u_{j}(t, x), & x \in \omega_{j}, t>0, \\ z_{j}(0, \rho, x)=\left.u_{j}\right|_{\omega_{j}}\left(-h_{j} \rho, x\right)=z_{j}^{0}\left(-h_{j} \rho, x\right), \rho \in(0,1) .\end{cases}
$$

Define $\mathfrak{h}=\max _{j=1, \cdots N} h_{j}$. 
Proposition 2.2 Assume $\underline{a}, \underline{b} \in \mathbb{L}^{\infty}(\mathcal{T})$ componentwise non-negative that satisfy (1.1) and $(1.2)$. Let $\left(U_{0}, g\right) \in \bar{D}(\mathcal{A}) \times C_{0}^{2}([0, T])$ where $C_{0}^{2}([0, T]):=\left\{\varphi \in C^{2}([0, T])\right.$ : $\varphi(0)=0\}$. Then there exists a unique classical solution $U=(\underline{u} \underline{\underline{z}}) \in C([0, T], D(\mathcal{A})) \cap$ $C^{1}([0, T] ; H)$ of 2.17$)-2.18$.

Proof Let $\underline{v}=\underline{u}-g \underline{\phi}$, where $\underline{\phi}$ is defined as

$$
\phi_{j}(x)=\frac{\left(x-\ell_{j}\right)^{2}}{\ell_{j}^{2}\left(2 \sum_{j=1}^{N} \ell_{j}^{-2}+\alpha\right)}
$$

We can easily check that

$$
\left\{\begin{array}{lc}
\phi_{j}\left(\ell_{j}\right)=\phi_{j}^{\prime}\left(\ell_{j}\right)=0, & \forall j=1, \cdots, N \\
\phi_{j}(0)=\frac{1}{2 \sum_{j=1}^{N} \ell_{j}^{-2}+\alpha}=\phi_{k}(0), \forall j, k=1, \cdots N, \\
\sum_{j=1}^{N} \phi_{j}^{\prime \prime}(0)=1-\alpha \phi_{1}(0), & t>0 .
\end{array}\right.
$$

We extend $g$ on $[-\mathfrak{h}, 0]$ by $g(t) \equiv 0$ for $t \in[-\mathfrak{h}, 0]$. Then $\underline{v}$ satisfies

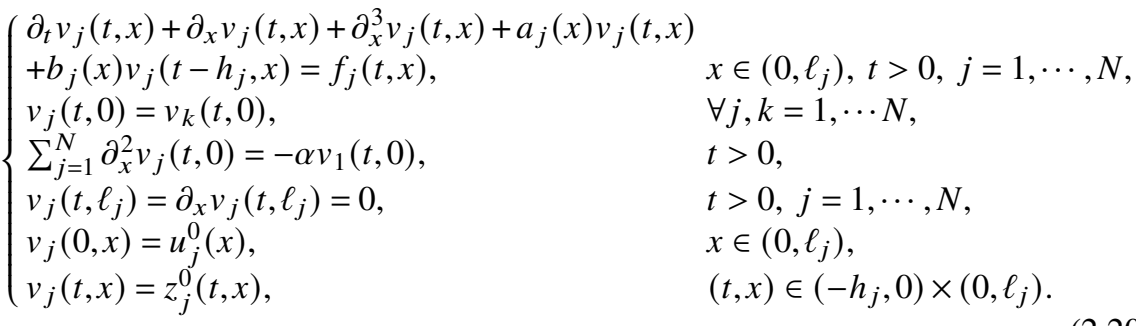

for $f_{j}(t, x)=-\phi_{j}(x) g^{\prime}(t)-\left(\phi_{j}^{\prime}+\phi_{j}^{\prime \prime \prime}+a_{j} \phi_{j}\right)(x) g(t)$. Then, taking $y_{j}(t, \rho, x)=\left.v_{j}\right|_{\omega_{j}}(t-$ $\left.h_{j} \rho, x\right)$

$$
\begin{cases}h_{j} \partial_{t} y_{j}(t, \rho, x)+\partial_{\rho} y_{j}(t, \rho, x)=0, & x \in \omega_{j}, \rho \in(0,1), t>0 \\ y_{j}(t, 0, x)=v_{j}(t, x), & x \in \omega_{j}, t>0 \\ y_{j}(0, \rho, x)=\left.v_{j}\right|_{\omega_{j}}\left(-h_{j} \rho, x\right)=z_{j}^{0}\left(-h_{j} \rho, x\right), \rho \in(0,1)\end{cases}
$$

Thus defining $V=\left(\frac{v}{y}\right)$, as $-\underline{\phi} g^{\prime}-\left(\underline{\phi}^{\prime}+\underline{\phi}^{\prime \prime \prime}+\underline{a} . * \underline{\phi}\right) g \in C^{1}\left([0, T], \mathbb{L}^{2}(\mathcal{T})\right)$, by the classical semigroup theory and the well-posedness of the linear case, we deduce the existence of a unique solution $V$ of 2.20$]$ - 2.21). Moreover $V \in C([0, T], D(\mathcal{A})) \cap$ $C^{1}([0, T] ; H)$ and hence 2.17)-2.18 admits a unique solution $U \in C([0, T], D(\mathcal{A})) \cap$ $C^{1}([0, T] ; H)$. 
Now, we study the same system but with less regular data.

Proposition 2.3 Assume $\underline{a}, \underline{b} \in \mathbb{L}^{\infty}(\mathcal{T})$ componentwise non-negative that satisfy $(1.1)$ and (1.2). Let $\left(U_{0}, g\right) \in \bar{H} \times L^{2}(0, T)$, then there exists a unique mild solution $U \in$ $\mathbb{B} \times C\left([0, T] ; \mathbb{L}^{2}(\Omega)\right)$ of 2.17$)-\left(2.18\right.$. Furthermore $u_{1}(\cdot, 0)$ and $\partial_{x} \underline{u}(\cdot, 0)$ belong to $L^{2}(0, T)$ and we have the following estimates

$$
\begin{gathered}
\|\underline{u}\|_{\mathbb{B}}^{2} \leq C\left(\|g\|_{L^{2}(0, T)}^{2}+\left\|\underline{u}^{0}\right\|_{\mathbb{L}^{(\mathcal{T})}}^{2}+\left\|\underline{z}^{0}(-\underline{h} \cdot, \cdot)\right\|_{\mathbb{L}^{2}(\Omega)}^{2}\right) . \\
\|\underline{z}\|_{C\left([0, T], \mathbb{L}^{2}(\Omega)\right)}^{2} \leq C\left(\left\|\underline{u}^{0}\right\|_{\mathbb{L}^{2}(\mathcal{T})}^{2}+\left\|\underline{z}^{0}(-\underline{h} \cdot, \cdot)\right\|_{\mathbb{L}^{2}(\Omega)}^{2}+\|g\|_{L^{2}(0, T)}^{2}\right) . \\
\left\|\underline{u}^{0}\right\|_{\mathbb{L}^{2}(\mathcal{T})}^{2} \leq\left(\frac{1+2 T\|\underline{a}\|_{\mathbb{L}^{\infty}(\mathcal{T})}+2 T\|\underline{b}\|_{\mathbb{L}^{\infty}(\mathcal{T})}}{T}\|\underline{u}\|_{L^{2}\left(0, T ; \mathbb{L}^{2}(\mathcal{T})\right)}^{2}\right. \\
+\|\underline{b}\|_{\mathbb{L}^{\infty}(\mathcal{T})}\left\|\underline{z}^{0}(-\underline{h} \cdot, \cdot)\right\|_{\mathbb{L}^{2}(\Omega)}^{2}+C\left(\left\|u_{1}(\cdot, 0)\right\|_{L^{2}(0, T)}^{2}+\|g\|_{L^{2}(0, T)}^{2}\right) \\
\left\|\underline{z}^{0}(-\underline{h}, \cdot)\right\|_{\mathbb{L}^{2}(\Omega)}^{2} \leq\|\underline{z}(T, \cdot, \cdot)\|_{\mathbb{L}^{2}(\Omega)}^{2}+\sum_{j=1}^{N} \frac{1}{h_{j}} \int_{0}^{T} \int_{\omega_{j}}\left|z_{j}(t, 1, x)\right|^{2} d x d t .
\end{gathered}
$$

Proof Those estimates are obtained in a similar way as Proposition 2.1 and for that many calculations are omitted. First suppose that $\left(U_{0}, g\right) \in D(\mathcal{A}) \times C_{0}^{2}([0, T])$ and thus the solution of 2.17-2.18 satisfies $U \in C\left([0, T] ; D(\mathcal{A}) \cap C^{1}([0, T] ; H)\right.$.

Multiplying 2.17) by $u_{j}$ and integrating on $[0, s] \times\left[0, \ell_{j}\right]$ gives us

$$
\begin{gathered}
\sum_{j=1}^{N} \int_{0}^{\ell_{j}}\left|u_{j}(s, x)\right|^{2} d x+\int_{0}^{s} \sum_{j=1}^{N}\left|\partial_{x} u_{j}(t, 0)\right|^{2} d t+(2 \alpha-N) \int_{0}^{s}\left|u_{1}(t, 0)\right|^{2} d t \\
+2 \sum_{j=1}^{N} \int_{0}^{s} \int_{0}^{\ell_{j}} a_{j}\left|u_{j}\right|^{2} d x d t+2 \sum_{j=1}^{N} \int_{0}^{s} \int_{0}^{\ell_{j}} b_{j} u_{j}\left(t-h_{j}, x\right) u_{j}(t, x) d x d t \\
=\sum_{j=1}^{N} \int_{0}^{\ell_{j}}\left|u_{j}(0, x)\right|^{2} d x+2 \int_{0}^{s} u_{1}(t, 0) g(t) d t,
\end{gathered}
$$

then using that

$$
\begin{gathered}
2 \int_{0}^{s} \int_{0}^{\ell_{j}} b_{j}(x) u_{j}\left(t-h_{j}, x\right) u_{j}(t, x) d x d t \\
\leq 2 \int_{0}^{s} \int_{0}^{\ell_{j}} b_{j}(x)\left|u_{j}(t, x)\right|^{2} d x d t+\int_{-h_{j}}^{0} \int_{\omega_{j}} b_{j}(x)\left|z_{j}^{0}(t, x)\right|^{2} d x d t \\
\sum_{j=1}^{N} \int_{0}^{\ell_{j}}\left|u_{j}(s, x)\right|^{2} d x+\int_{0}^{s} \sum_{j=1}^{N}\left|\partial_{x} u_{j}(t, 0)\right|^{2} d t+(2 \alpha-N) \int_{0}^{s}\left|u_{1}(t, 0)\right|^{2} d t+ \\
2 \sum_{j=1}^{N} \int_{0}^{s} \int_{0}^{\ell_{j}}\left(a_{j}-b_{j}\right)\left|u_{j}\right|^{2} d x d t \leq 2 \int_{0}^{s} u_{1}(t, 0) g(t) d t \\
+C\left(\left\|\underline{u}^{0}\right\|_{\mathbb{L}(\mathcal{T})}^{2}+\left\|\underline{z}^{0}(-\underline{h} \cdot, \cdot)\right\|_{\mathbb{L}^{2}(\Omega)}^{2}\right) .
\end{gathered}
$$


Note now that 2.16 still holds in this case and we can obtain

$$
\begin{gathered}
\left\|u_{1}(\cdot, 0)\right\|_{L^{2}(0, T)}^{2} \leq C\left(\|\underline{u}\|_{L^{2}\left(0, T ; \mathbb{L}^{2}(\mathcal{T})\right)}^{2}+\left\|\underline{u}^{0}\right\|_{\mathbb{L}^{2}(\mathcal{T})}^{2}+\sum_{j=1}^{N} \int_{0}^{T}\left|\partial_{x} u_{j}(t, 0)\right|^{2} d t\right. \\
\left.+\left\|\underline{z}^{0}(-\underline{h} \cdot, \cdot)\right\|_{\mathbb{L}^{2}(\Omega)}^{2}\right) .
\end{gathered}
$$

From 2.26 we obtain

$$
\left\|u_{1}(\cdot, 0)\right\|_{L^{2}(0, T)}^{2} \leq C\left(\left\|\underline{u}^{0}\right\|_{\mathbb{L}^{2}(\mathcal{T})}^{2}+\left\|\underline{z}^{0}(-\underline{h} \cdot, \cdot)\right\|_{\mathbb{L}^{2}(\Omega)}^{2}+\int_{0}^{T} u_{1}(t, 0) g(t) d t\right)
$$

and again by Young's inequality

$$
\left\|u_{1}(\cdot, 0)\right\|_{L^{2}(0, T)}^{2} \leq C\left(\left\|\underline{u}^{0}\right\|_{\mathbb{L}^{2}(\mathcal{T})}^{2}+\left\|\underline{z}^{0}(-\underline{h} \cdot, \cdot)\right\|_{\mathbb{L}^{2}(\Omega)}^{2}+\|g\|_{L^{2}(0, T)}^{2}\right)
$$

Thus $u_{1}(\cdot, 0) \in L^{2}(0, T)$ and from $[2.26) \underline{u}(s, \cdot) \in \mathbb{L}^{2}(\mathcal{T})$ for $s \in[0, T], \partial_{x} \underline{u}(\cdot, 0) \in$ $L^{2}(0, T)$ and

$$
\max _{s \in[0, T]}\|\underline{u}(s, \cdot)\|_{\mathbb{L}^{2}(\mathcal{T})}^{2} \leq C\left(\|g\|_{L^{2}(0, T)}^{2}+\left\|\underline{u}^{0}\right\|_{\mathbb{L}^{2}(\mathcal{T})}^{2}+\left\|\underline{z}^{0}(-\cdot \underline{h} \cdot \cdot)\right\|_{\mathbb{L}^{2}(\Omega)}^{2}\right) .
$$

Now multiplying 2.17) by $x u_{j}$ yields

$$
\begin{aligned}
& \left.\int_{0}^{\ell_{j}} x\left|u_{j}\right|^{2} d x\right|_{0} ^{T} d x-\int_{0}^{T} \int_{0}^{\ell_{j}}\left|u_{j}\right|^{2} d x d t+2 \int_{0}^{T} \int_{0}^{\ell_{j}} x b_{j}(x) u_{j}\left(t-h_{j}, x\right) u_{j}(t, x) d x d t \\
& +2 \int_{0}^{T} \int_{0}^{\ell_{j}} a_{j}(x) x\left|u_{j}\right|^{2} d x d t+3 \int_{0}^{T} \int_{0}^{\ell_{j}}\left|\partial_{x} u_{j}\right|^{2} d x d t=\int_{0}^{T}-2 u_{j}(t, 0) \partial_{x} u_{j}(t, 0) d t .
\end{aligned}
$$

Then

$$
\begin{gathered}
3 \sum_{j=1}^{N} \int_{0}^{T} \int_{0}^{\ell_{j}}\left|\partial_{x} u_{j}\right|^{2} d x d t \leq\left(1+2 L\|\underline{b}\|_{L^{\infty}(\mathcal{T})}\right) \sum_{j=1}^{N} \int_{0}^{T} \int_{0}^{\ell_{j}}\left|u_{j}\right|^{2} d x d t \\
+L \sum_{j=1}^{N} \int_{0}^{\ell_{j}}\left|u_{j}(0, x)\right|^{2} d x+L\|\underline{b}\|_{L^{\infty}(\mathcal{T})} \sum_{j=1}^{N} \int_{-h_{j}}^{0} \int_{\omega_{j}}\left|z_{j}^{0}(t, x)\right|^{2} d x d t \\
+\left\|u_{1}(\cdot, 0)\right\|_{L^{2}(0, T)}^{2}+\left\|\partial_{x} \underline{u}(\cdot, 0)\right\|_{L^{2}(0, T)}^{2}
\end{gathered}
$$

and using 2.27) we deduce 2.22). Now multiplying 2.17) by $(T-t) u_{j}$ yields

$$
\begin{gathered}
-\int_{0}^{\ell_{j}} T\left|u_{j}(0, x)\right|^{2} d x+\int_{0}^{T} \int_{0}^{\ell_{j}}\left|u_{j}\right|^{2} d x d t+2 \int_{0}^{T} \int_{0}^{\ell_{j}} a_{j}(x)(T-t)\left|u_{j}\right|^{2} d x d t \\
+2 \int_{0}^{T} \int_{0}^{\ell_{j}} b_{j}(x)(T-t) u_{j}\left(t-h_{j}, x\right) u_{j}(t, x) d x d t=\int_{0}^{T}\left[(T-t)\left|u_{j}(t, 0)\right|^{2}\right. \\
\left.+2(T-t) u_{j}(t, 0) \partial_{x}^{2} u_{j}(t, 0)-(T-t)\left|\partial_{x} u_{j}(t, 0)\right|^{2}\right] d t,
\end{gathered}
$$


then

$$
\begin{gathered}
T \sum_{j=1}^{N} \int_{0}^{\ell_{j}}\left|u_{j}(0, x)\right|^{2} d x=\sum_{j=1}^{N}\left(\int_{0}^{T} \int_{0}^{\ell_{j}}\left|u_{j}\right|^{2} d x d t+2 \int_{0}^{T} \int_{0}^{\ell_{j}}(T-t) a_{j}\left|u_{j}\right|^{2} d x d t+\right. \\
\left.2 \int_{0}^{T} \int_{0}^{\ell_{j}} b_{j}(x)(T-t) u_{j}\left(t-h_{j}, x\right) u_{j}(t, x) d x d t\right)+(2 \alpha-N) \int_{0}^{T}(T-t)\left|u_{1}(t, 0)\right|^{2} d t \\
+\sum_{j=1}^{N} \int_{0}^{T}(T-t)\left|\partial_{x} u_{j}(t, 0)\right|^{2} d t-2 \int_{0}^{T}(T-t) u_{1}(t, 0) g(t) d t .
\end{gathered}
$$

Finally we get

$$
\begin{aligned}
& \|\underline{u}\|_{\mathbb{L}^{2}(\mathcal{T})}^{2} \leq\left(\frac{1+2 T\|\underline{a}\|_{\mathbb{L}^{\infty}(\mathcal{T})}+2 T\|\underline{b}\|_{\mathbb{L}^{\infty}(\mathcal{T})}}{T}\right)\|\underline{u}\|_{L^{2}\left(0, T ; \mathbb{L}^{2}(\mathcal{T})\right)}^{2} \\
& +\|\underline{b}\|_{\mathbb{L}^{\infty}(\mathcal{T})}\left\|\underline{z}^{0}(-\underline{h} \cdot, \cdot)\right\|_{\mathbb{L}^{2}(\Omega)}^{2}+C\left(\left\|u_{1}(\cdot, 0)\right\|_{L^{2}(0, T)}^{2}+\|g\|_{L^{2}(0, T)}^{2}\right)
\end{aligned}
$$

and hence (2.24). We can conclude that the estimates for 2.18 are the same as Proposition 2.1. By density of $D(\mathcal{A})$ in $H, C_{0}^{2}([0, T])$ in $L^{2}(0, T)$, we extend our result to arbitrary data $\left(U_{0}, g\right) \in H \times L^{2}(0, T)$.

\subsection{Extra source term}

We add now a source term $f_{j}(t, x)$ on each edge in our $\mathrm{KdV}$ problem.

$$
\begin{cases}\partial_{t} u_{j}(t, x)+\partial_{x} u_{j}(t, x)+\partial_{x}^{3} u_{j}(t, x)+a_{j}(x) u_{j}(t, x) & x \in\left(0, \ell_{j}\right), t>0, j=1, \cdots, N, \\ +b_{j}(x) u_{j}\left(t-h_{j}, x\right)=f_{j}(t, x), & \forall j, k=1, \cdots N, \\ u_{j}(t, 0)=u_{k}(t, 0), & t>0, \\ \sum_{j=1}^{N} \partial_{x}^{2} u_{j}(t, 0)=-\alpha u_{1}(t, 0)+g(t), & t>0, j=1, \cdots, N, \\ u_{j}\left(t, \ell_{j}\right)=\partial_{x} u_{j}\left(t, \ell_{j}\right)=0, & x \in\left(0, \ell_{j}\right) \\ u_{j}(0, x)=u_{j}^{0}(x), & (t, x) \in\left(-h_{j}, 0\right) \times\left(0, \ell_{j}\right) .\end{cases}
$$

We set as in the previous cases $z_{j}(t, \rho, x)=\left.u_{j}\right|_{\omega_{j}}\left(t-h_{j} \rho, x\right) x \in \omega_{j}, \rho \in(0,1)$. Then

$$
\begin{cases}h_{j} \partial_{t} z_{j}(t, \rho, x)+\partial_{\rho} z_{j}(t, \rho, x)=0, & x \in \omega_{j}, \rho \in(0,1), t>0 \\ z_{j}(t, 0, x)=u_{j}(t, x), & x \in \omega_{j}, t>0 \\ z_{j}(0, \rho, x)=\left.u_{j}\right|_{\omega_{j}}\left(-h_{j} \rho, x\right)=z_{j}^{0}\left(-h_{j} \rho, x\right), \rho \in(0,1)\end{cases}
$$

Proposition 2.4 Assume $\underline{a}, \underline{b} \in \mathbb{L}^{\infty}(\mathcal{T})$ componentwise non-negative that satisfy (1.1) and (1.2). Let $\left(U_{0}, g, f\right) \in H \times L^{2}(0, T) \times L^{1}\left(0, T ; \mathbb{L}^{2}(\mathcal{T})\right)$ then there exists a unique mild solution $U=\left(\frac{u}{\underline{z}}\right) \in \mathbb{B} \times C\left([0, T] ; \mathbb{L}^{2}(\Omega)\right)$ to 2.28$)$-2.29]. Furthermore we have the following estimates, 


$$
\begin{gathered}
\|(\underline{u}, \underline{z})\|_{C([0, T], H)}^{2} \leq C\left(\left\|\underline{u}^{0}\right\|_{\mathbb{L}^{2}(\mathcal{T})}^{2}+\left\|\underline{z}^{0}(-\underline{h}, \cdot)\right\|_{\mathbb{L}^{2}(\Omega)}^{2}+\|\underline{f}\|_{L^{1}\left(0, T ; \mathbb{L}^{2}(\mathcal{T})\right)}^{2}+\|g\|_{L^{2}(0, T)}^{2}\right), \\
\left\|\partial_{x} \underline{u}\right\|_{L^{2}\left(0, T ; \mathbb{L}^{2}(\mathcal{T})\right)}^{2} \leq C(1+T)\left(\left\|\underline{u}^{0}\right\|_{\mathbb{L}^{2}(\mathcal{T})}^{2}+\left\|\underline{z}^{0}(-\underline{h} \cdot, \cdot)\right\|_{\mathbb{L}^{2}(\Omega)}^{2}\right. \\
\left.+\|f\|_{L^{1}\left(0, T ; \mathbb{L}^{2}(\mathcal{T})\right)}^{2}+\|g\|_{L^{2}(0, T)}^{2}\right) .
\end{gathered}
$$

Proof The well-posedness of 2.28)-2.29] follows from classical semigroup theory and from the propositions given considering the source term $\left(\frac{f}{\underline{0}}\right)$. Also this gives us the first inequality, for the second one note that multiplying (2.28) by $u_{j}$ and integrating we get

$$
\begin{gathered}
\sum_{j=1}^{N} \int_{0}^{\ell_{j}}\left|u_{j}(T, x)\right|^{2} d x+(2 \alpha-N) \int_{0}^{T}\left|u_{1}(t, 0)\right|^{2} d t+\sum_{j=1}^{N} \int_{0}^{T}\left|\partial_{x} u_{j}(t, 0)\right|^{2} d t \\
+2 \sum_{j=1}^{N} \int_{0}^{T} \int_{0}^{\ell_{j}} a_{j}(x)\left|u_{j}(t, x)\right|^{2} d x d t+2 \sum_{j=1}^{N} \int_{0}^{T} \int_{0}^{\ell_{j}} b_{j} u_{j}(t, x) u_{j}\left(t-h_{j}, x\right) d x d t \\
-2 \int_{0}^{T} u_{1}(t, 0) g(t) d t=2 \sum_{j=1}^{N} \int_{0}^{T} \int_{0}^{\ell_{j}} f_{j}(t, x) u_{j}(t, x) d x d t+\left\|\underline{u}^{0}\right\|_{\mathbb{L}^{2}(\mathcal{T})}^{2} .
\end{gathered}
$$

Note that

$$
\begin{gathered}
2 \sum_{j=1}^{N} \int_{0}^{T} \int_{0}^{\ell_{j}} f_{j}(t, x) u_{j}(t, x) d x d t \leq 2 \sum_{j=1}^{N} \int_{0}^{T}\left\|f_{j}\right\|_{L^{2}\left(0, \ell_{j}\right)}\left\|u_{j}\right\|_{L^{2}\left(0, \ell_{j}\right)} d t, \\
\leq 2 \sum_{j=1}^{N}\left\|u_{j}\right\|_{C\left([0, T], L^{2}\left(0, \ell_{j}\right)\right)} \int_{0}^{T}\left\|f_{j}\right\|_{L^{2}\left(0, \ell_{j}\right)} d t \leq\|\underline{u}\|_{C\left([0, T], \mathbb{L}^{2}(\mathcal{T})\right)}^{2}+\|\underline{f}\|_{L^{1}\left(0, T ; \mathbb{L}^{2}(\mathcal{T})\right)}^{2} .
\end{gathered}
$$

Following the same steps as in Proposition 2.1 and Proposition 2.3 we can get

$$
\begin{gathered}
\sum_{j=1}^{N} \int_{0}^{\ell_{j}}\left|u_{j}(T, x)\right|^{2} d x+\sum_{j=1}^{N} \int_{0}^{T}\left|\partial_{x} u_{j}(t, 0)\right|^{2} d t \leq C\left(\|\underline{u}\|_{L^{2}\left(0, T ; \mathbb{L}^{2}(\mathcal{T})\right)}^{2}+\left\|\left(\underline{u}^{0}, \underline{z}^{0}(-\underline{h} \cdot, \cdot)\right)\right\|_{H}^{2}\right. \\
\left.+2 \int_{0}^{T} u_{1}(t, 0) g(t) d t+\|\underline{u}\|_{C\left([0, T], \mathbb{L}^{2}(\mathcal{T})\right)}^{2}+\|\underline{f}\|_{L^{1}\left(0, T ; \mathbb{L}^{2}(\mathcal{T})\right)}^{2}\right) .
\end{gathered}
$$

Now multiplying (2.28) by $q_{j} u_{j}$ for $q_{j}=\frac{x\left(2 \ell_{j}-x\right)}{\ell_{j}^{2}}$ and using the last inequality we get $\left\|u_{1}(\cdot, 0)\right\|_{L^{2}(0, T)}^{2} \leq C\left(\left\|\underline{u}^{0}\right\|_{\mathbb{L}^{2}(\mathcal{T})}^{2}+\left\|\underline{z}^{0}(-\underline{h} \cdot, \cdot)\right\|_{\mathbb{L}^{2}(\Omega)}^{2}+\|g\|_{L^{2}(0, T)}^{2}+\|\underline{f}\|_{L^{1}\left(0, T ; \mathbb{L}^{2}(\mathcal{T})\right)}^{2}\right)$ and we can also have

$$
\begin{gathered}
\left\|u_{1}(\cdot, 0)\right\|_{L^{2}(0, T)}^{2}+\left\|\partial_{x} u(\cdot, 0)\right\|_{L^{2}(0, T)}^{2} \leq C\left(\|\underline{f}\|_{L^{1}\left(0, T ; \mathbb{L}^{2}(\mathcal{T})\right)}^{2}+\|g\|_{L^{2}(0, T)}^{2}\right. \\
\left.+\left\|\left(\underline{u}^{0}, \underline{z}^{0}(-\underline{h}, \cdot)\right)\right\|_{H}^{2}\right) .
\end{gathered}
$$


Now multiplying 2.28 by $x u_{j}$ gives us

$$
\begin{gathered}
3 \sum_{j=1}^{N} \int_{0}^{T} \int_{0}^{\ell_{j}}\left|\partial_{x} u_{j}\right|^{2} d x d t+\sum_{j=1}^{N} \int_{0}^{\ell_{j}} x\left|u_{j}(T, x)\right|^{2} d x-\sum_{j=1}^{N} \int_{0}^{T} \int_{0}^{\ell_{j}}\left|u_{j}\right|^{2} d x d t \\
+2 \sum_{j=1}^{N} x\left|u_{j}\right|^{2} a_{j} d x d t+2 \sum_{j=1}^{N} \int_{0}^{T} \int_{0}^{\ell_{j}} x b_{j} u_{j}\left(t-h_{j}, x\right) u_{j}(t, x) d x d t \\
\quad=2 \sum_{j=1}^{N} \int_{0}^{T} \int_{0}^{\ell_{j}} x u_{j} f_{j} d x d t-2 \sum_{j=1}^{N} \int_{0}^{T} u_{1}(t, 0) \partial_{x} u_{j}(t, 0) d t .
\end{gathered}
$$

Hence

$$
\begin{aligned}
3\left\|\partial_{x} \underline{u}\right\|_{L^{2}\left(0, T ; \mathbb{L}^{2}(\mathcal{T})\right)}^{2} \leq & T\|\underline{u}\|_{C\left([0, T] ; \mathbb{L}^{2}(\mathcal{T})\right)}^{2}+L\left(\|\underline{u}\|_{C\left([0, T], \mathbb{L}^{2}(\mathcal{T})\right)}^{2}+\|\underline{f}\|_{L^{1}\left(0, T ; \mathbb{L}^{2}(\mathcal{T})\right)}^{2}\right) \\
& +N\left\|u_{1}(\cdot, 0)\right\|_{L^{2}(0, T)}^{2}+\left\|\partial_{x} \underline{u}(\cdot, 0)\right\|_{L^{2}(0, T)}^{2} .
\end{aligned}
$$

2.4 Well-posedness of nonlinear system

The aim of this section is to use the estimates obtained in the last sections to pass to the nonlinear system. The following propositions are needed in order to deal with the internal nonlinearity and boundary nonlinearity respectively.

Proposition 2.5 (Proposition 4.1, [18]) Let $T, L>0$, and $y \in L^{2}\left(0, T ; H^{1}(0, L)\right.$ ). Then $y y_{x} \in L^{1}\left(0, T ; L^{2}(0, L)\right)$ and the map

$$
y \in L^{2}\left(0, T ; H^{1}(0, L)\right) \mapsto y y_{x} \in L^{1}\left(0, T ; L^{2}(0, L)\right)
$$

is continuous. Moreover we have

$$
\left\|y y_{x}\right\|_{L^{1}\left(0, T ; L^{2}(0, L)\right)} \leq C\|y\|_{L^{2}\left(0, T ; H^{1}(0, L)\right)}^{2} .
$$

Proposition 2.6 (Proposition 2.6, [1]) Let $\underline{u} \in \mathbb{B}$, then $\left|u_{1}(t, 0)\right|^{2} \in L^{2}(0, T)$ and the map

$$
\underline{u} \in \mathbb{B} \mapsto\left|u_{1}(t, 0)\right|^{2} \in L^{2}(0, T)
$$

is continuous. Moreover, we have the estimate,

$$
\left\|u_{1}^{2}(\cdot, 0)\right\|_{L^{2}(0, T)} \leq \frac{1}{\sqrt{2}}\|\underline{u}\|_{\mathbb{B}}^{2} .
$$

Now we are ready to establish our well-posedness result of the nonlinear $\mathrm{KdVd}$ for small initial data.

Theorem 2.2 Assume $\underline{a}, \underline{b} \in \mathbb{L}^{\infty}(\mathcal{T})$ componentwise non-negative that satisfy (1.1) and (1.2). Let $\left(\ell_{j}\right)_{j=1}^{N} \subset(0,+\infty), T>0$, there exists $\epsilon>0$ and $C>0$ such that for all $U_{0}=\left(\underline{u}^{0}, \underline{z}^{0}(-\underline{h} \cdot, \cdot)\right) \in H$ with $\left\|U_{0}\right\|_{H} \leq \epsilon$, the nonlinear equation $\mathrm{KdVd}$ has a unique mild solution $\underline{u} \in \mathbb{B}$. Moreover it satisfy

$$
\|\underline{u}\|_{\mathbb{B}} \leq C\left\|\left(\underline{u}^{0}, \underline{z}^{0}(-\underline{h} \cdot, \cdot)\right)\right\|_{H} .
$$


Proof Let $U_{0} \in H$, with $\left\|U_{0}\right\|_{H}<\epsilon$, where $\epsilon>0$ will be chosen later, $\underline{u} \in \mathbb{B}$ and consider the map $\Phi: \mathbb{B} \rightarrow \mathbb{B}$ defined by $\Phi(\underline{u})=\underline{v}$ where $\underline{v}$ is solution of

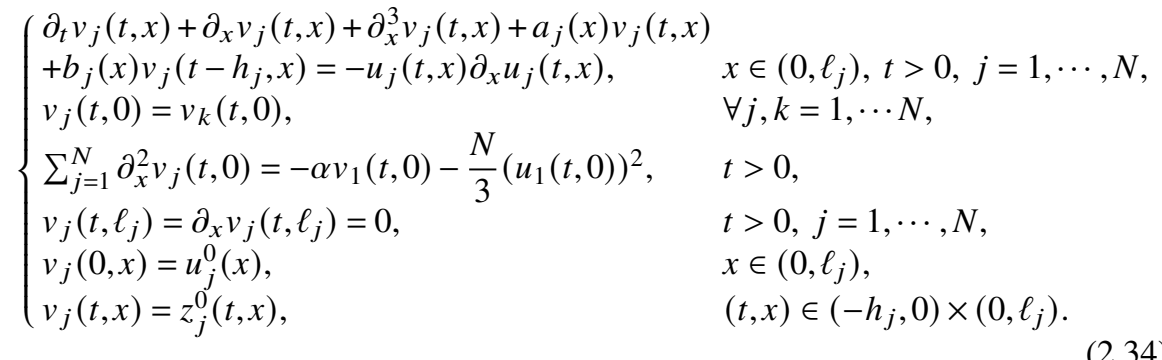

Clearly $\underline{u} \in \mathbb{B}$ is solution of $(\mathrm{KdVd})$ if $\underline{u}$ is a fixed point $\Phi$. From Proposition 2.5 and Proposition 2.6 we get for all $\underline{u} \in \mathbb{B}$

$$
\|\Phi(\underline{u})\|_{\mathbb{B}}=\|\underline{v}\|_{\mathbb{B}} \leq C\left(\left\|U_{0}\right\|_{H}+\|\underline{u}\|_{\mathbb{B}}^{2}\right)
$$

and for $\underline{u}, \underline{\tilde{u}} \in \mathbb{B}$

$$
\|\Phi(\underline{u})-\Phi(\underline{\tilde{u}})\|_{\mathbb{B}} \leq C\left(\|\underline{u}\|_{\mathbb{B}}+\|\underline{\tilde{u}}\|_{\mathbb{B}}\right)\|\underline{u}-\underline{\tilde{u}}\|_{\mathbb{B}}
$$

Let us choose $R>0$ to be defined later and consider $\Phi$ restricted to the closed ball $B_{\mathbb{B}}(0, R)$. Then, for any $\underline{u}, \underline{\tilde{u}} \in B_{\mathbb{B}}(0, R)$, we have

$$
\begin{gathered}
\|\Phi(\underline{u})\|_{\mathbb{B}} \leq C\left(\epsilon+R^{2}\right) \\
\|\Phi(\underline{u})-\Phi(\underline{\tilde{u}})\|_{\mathbb{B}} \leq 2 C R\|\underline{u}-\underline{\tilde{u}}\|_{\mathbb{B}} .
\end{gathered}
$$

Thus if $R<\frac{1}{2 C}$ and $\epsilon>0$ such that $C\left(\epsilon+R^{2}\right)<R$ we obtain the local well-posedness result applying the Banach fixed point Theorem.

Remark 2.1 On a similar way as Theorem 2.1, we can obtain classical solutions by taking $U_{0} \in D(\mathcal{A})$.

\section{Stabilization of delayed KdV system}

3.1 Lyapunov stabilization of the delayed system

The aim of this part is to prove Theorem 1.1 As we said before this proof is developed in a constructive manner by using a Lyapunov function. 
Proof of Theorem 1.1:

Let $\underline{u}$ a regular enough solution of $\overline{\mathrm{KdVd}}$ with $U_{0} \in D(\mathcal{A})$ satisfying $\left\|U_{0}\right\|_{H} \leq \epsilon$, where $\epsilon>0$ will be chosen later. Following [2, 21] we consider the next Lyapunov candidate for (KdVd)

$$
V(t)=E(t)+\mu_{1} V_{1}(t)+\mu_{2} V_{2}(t) .
$$

where $E$ is defined by 1.6

$V_{1}(t)=\sum_{j=1}^{N} \int_{0}^{\ell_{j}} x\left|u_{j}(t, x)\right|^{2} d x$, and $V_{2}(t)=\sum_{j=1}^{N} h_{j} \int_{\omega_{j}} \int_{0}^{1}(1-\rho)\left|u_{j}\left(t-h_{j} \rho, x\right)\right|^{2} d \rho d x$.

Clearly

$$
E(t) \leq V(t) \leq\left(1+\max \left\{L \mu_{1}, \frac{\mu_{2}}{b_{0}}\right\}\right) E(t) .
$$

After some computations we have,

$$
\begin{gathered}
\frac{\mathrm{d}}{\mathrm{d} t} E(t) \leq-(2 \alpha-N)\left|u_{1}(t, 0)\right|^{2}-\sum_{j=1}^{N}\left|\partial_{x} u_{j}(t, 0)\right|^{2}-\sum_{j=1}^{N} \int_{\left(0, \ell_{j}\right) / \omega_{j}} a_{j}(x)\left|u_{j}(t, x)\right|^{2} d x \\
+\sum_{j=1}^{N} \int_{\omega_{j}}\left(-2 a_{j}(x)+b_{j}(x)+\xi_{j}(x)\right)\left|u_{j}(t, x)\right|^{2} d x+\sum_{j=1}^{N} \int_{\omega_{j}}\left(b_{j}(x)-\xi_{j}(x)\right)\left|u_{j}\left(t-h_{j}, x\right)\right|^{2} d x, \\
\frac{\mathrm{d}}{\mathrm{d} t} V_{1}(t)=\sum_{j=1}^{N} \int_{0}^{\ell_{j}}\left|u_{j}(t, x)\right|^{2} d x-3 \sum_{j=1}^{N} \int_{0}^{\ell_{j}}\left|\partial_{x} u_{j}(t, x)\right|^{2} d x-2 u_{1}(t, 0) \sum_{j=1}^{N} \partial_{x} u_{j}(t, 0) \\
+\frac{2}{3} \sum_{j=1}^{N} \int_{0}^{\ell_{j}} u_{j}^{3}(t, x) d x-2 \sum_{j=1}^{N} \int_{0}^{\ell_{j}} x a_{j}(x)\left|u_{j}(t, x)\right|^{2} d x-2 \sum_{j=1}^{N} \int_{\omega_{j}} x b_{j}(x) u_{j}(t, x) u_{j}\left(t-h_{j}, x\right) d x \\
\leq \sum_{j=1}^{N} \int_{0}^{\ell_{j}}\left|u_{j}(t, x)\right|^{2} d x-3 \sum_{j=1}^{N} \int_{0}^{\ell_{j}}\left|\partial_{x} u_{j}(t, x)\right|^{2} d x+\frac{N}{2}\left|u_{1}(t, 0)\right|^{2}+\frac{1}{2} \sum_{j=1}^{N}\left|\partial_{x} u_{j}(t, 0)\right|^{2} \\
+\frac{2}{3} \sum_{j=1}^{N} \int_{0}^{\ell_{j}} u_{j}^{3}(t, x) d x+L \sum_{j=1}^{n} \int_{\omega_{j}} b_{j}(x)\left|u_{j}(t, x)\right|^{2} d x++L \sum_{j=1}^{n} \int_{\omega_{j}} b_{j}(x)\left|u_{j}\left(t-h_{j}, x\right)\right|^{2} d x,
\end{gathered}
$$

and

$$
\frac{\mathrm{d}}{\mathrm{d} t} V_{2}(t)=\sum_{j=1}^{N} \int_{\omega_{j}}\left|u_{j}(t, x)\right|^{2} d x-\sum_{j=1}^{N} \int_{\omega_{j}} \int_{0}^{1}\left|u_{j}\left(t-h_{j} \rho, x\right)\right|^{2} d \rho d x .
$$

Our idea now is to prove that for suitable choice of $\mu_{1}, \mu_{2}, \gamma>0$ we have that $\frac{\mathrm{d}}{\mathrm{d} t} V(t)+2 \gamma V(t) \leq 0$, which gives the exponential stability.

Using the following Poincare's inequality: If $y \in H^{1}(0, L)$ and $y(0)=0$ or $y(L)=0$, 
we have $\|y\|_{L^{2}(0, L)} \leq \frac{2 L}{\pi}\left\|\partial_{x} y\right\|_{L^{2}(0, L)}$. We can check easily that for $\gamma>0$

$$
\begin{gathered}
\frac{\mathrm{d}}{\mathrm{d} t} V(t)+2 \gamma V(t) \leq-2\left(\alpha-\frac{N}{2}-\mu_{1} \frac{N}{2}\right)\left|u_{1}(t, 0)\right|^{2}+\left(\mu_{1}-1\right) \sum_{j=1}^{N}\left|\partial_{x} u_{j}(t, 0)\right|^{2} \\
+\sum_{j=1}^{N} \int_{\omega_{j}}\left(-2 a_{j}+b_{j}+\xi_{j}+L \mu_{1} b_{j}+\mu_{2}\right)\left|u_{j}\right|^{2} d x+\frac{2}{3} \mu_{1} \sum_{j=1}^{N} \int_{0}^{\ell_{j}}\left(u_{j}\right)^{3} d x \\
\quad+\sum_{j=1}^{N} \int_{\omega_{j}}\left(b_{j}-\xi_{j}+\mu_{1} L b_{j}\right)\left|u_{j}\left(t-h_{j}, x\right)\right|^{2} d x \\
+\left[\frac{4 L^{2}\left(\mu_{1}+2 \mu_{1} \gamma L+2 \gamma\right)}{\pi^{2}}-3 \mu_{1}\right] \sum_{j=1}^{N} \int_{0}^{\ell_{j}}\left|\partial_{x} u_{j}(t, x)\right|^{2} d x \\
+\sum_{j=1}^{N} \int_{\omega_{j}} \int_{0}^{1}\left(2 \gamma \mu_{2} h_{j}+2 \gamma h_{j} \xi_{j}-\mu_{2}\right)\left|u_{j}\left(t-h_{j} \rho, x\right)\right|^{2} d \rho d x .
\end{gathered}
$$

For the term involving $\int_{0}^{\ell_{j}} u_{j}^{3}(t, x) d x$, note that

$$
\int_{0}^{\ell_{j}} u_{j}^{3}(t, x) d x \leq\left\|u_{j}\right\|_{L^{\infty}\left(0, \ell_{j}\right)}^{2} \int_{0}^{\ell_{j}}\left|u_{j}(t, x)\right| d x \leq\left\|u_{j}\right\|_{L^{\infty}\left(0, \ell_{j}\right)}^{2}\left\|u_{j}\right\|_{L^{2}\left(0, \ell_{j}\right)} \sqrt{\ell_{j}} .
$$

By the injection of $H^{1}\left(0, \ell_{j}\right)$ into $L^{\infty}\left(0, \ell_{j}\right)$ we know that $\left\|u_{j}\right\|_{L^{\infty}\left(0, \ell_{j}\right)} \leq$ $\sqrt{\ell_{j}}\left\|\partial_{x} u_{j}\right\|_{L^{2}\left(0, \ell_{j}\right)}$, then

$$
\int_{0}^{\ell_{j}} u_{j}^{3}(t, x) d x \leq\left\|u_{j}\right\|_{L^{\infty}\left(0, \ell_{j}\right)}^{2}\left\|u_{j}\right\|_{L^{2}\left(0, \ell_{j}\right)} \sqrt{\ell_{j}} \leq \ell_{j}\left\|\partial_{x} u_{j}\right\|_{L^{2}\left(0, \ell_{j}\right)}^{2} \sqrt{\ell_{j}}\left\|u_{j}\right\|_{L^{2}\left(0, \ell_{j}\right)} .
$$

Recalling that $L=\max _{j=1, \cdots, N} \ell_{j}$ and as the energy is not increasing we get $\left\|u_{j}\right\|_{L^{2}\left(0, \ell_{j}\right)} \leq\left\|U_{0}\right\|_{H}$. Choosing $\left\|U_{0}\right\|_{H} \leq \epsilon$ we get

$$
\frac{2}{3} \mu_{1} \sum_{j=1}^{N} \int_{0}^{\ell_{j}} u_{j}^{3}(t, x) d x \leq \frac{2}{3} \mu_{1} \epsilon L^{3 / 2} \sum_{j=1}^{N} \int_{0}^{\ell_{j}}\left|\partial_{x} u_{j}(t, x)\right|^{2} d x .
$$

Now taking

$$
\begin{aligned}
0<\mu_{1}<\min _{j=1, \cdots, N} \inf _{\omega_{j}}\left\{1, \frac{2 a_{j}-b_{j}-\xi_{j}}{L b_{j}}, \frac{\xi_{j}-b_{j}}{L b_{j}}, \frac{1}{N}(2 \alpha-N)\right\}, \\
0<\mu_{2}<\min _{j=1, \cdots, N} \inf _{\omega_{j}}\left\{2 a_{j}-b_{j}-\xi_{j}-\mu_{1} L b_{j}\right\} .
\end{aligned}
$$

Then $-\left(2 \alpha-N-\mu_{1} N\right)<0$ and $\left(\mu_{1}-1\right)<0$. Moreover for all $j=1, \cdots, N$

$$
\left(-2 a_{j}+b_{j}+\xi_{j}+L \mu_{1} b_{j}+\mu_{2}\right)<0, \quad\left(b_{j}-\xi_{j}+\mu_{1} L b_{j}\right)<0
$$

Finally joining the estimates 


$$
\begin{gathered}
\frac{\mathrm{d}}{\mathrm{d} t} V(t)+2 \gamma V(t) \leq\left[\frac{4 L^{2}\left(\mu_{1}+2 \mu_{1} \gamma L+2 \gamma\right)}{\pi^{2}}-3 \mu_{1}+\frac{2 L^{3 / 2} \epsilon \mu_{1}}{3}\right]\left\|\partial_{x} \underline{u}(t, x)\right\|_{\mathbb{L}^{2}(\mathcal{T})}^{2} \\
+\sum_{j=1}^{N} \int_{\omega_{j}} \int_{0}^{1}\left(2 h_{j} \gamma\left(\mu_{2}+\xi_{j}\right)-\mu_{2}\right)\left|u_{j}\left(t-h_{j} \rho, x\right)\right|^{2} d \rho d x
\end{gathered}
$$

and then as $L<\frac{\sqrt{3}}{2} \pi$, we can choose $\epsilon<\frac{3}{2} \frac{\left(3 \pi^{2}-4 L^{2}\right)}{\pi^{2} L^{3 / 2}}$ and then take $\gamma>0$ satisfying (1.7) to obtain $\frac{\mathrm{d}}{\mathrm{d} t} V(t)+2 \gamma V(t) \leq 0$. We get the desired exponential stability, by density we can extend the result to any $U_{0} \in H$, with $\left\|U_{0}\right\|_{H} \leq \epsilon$.

Remark 3.1 As in [2, 21] we obtain an estimation of the rate of decay. Also recall that we can improve the result searching for a better Poincaré's inequality, and as is commented in [2, 21] looking for a new multiplier for the Lyapunov function $V_{1}$, in the sense that the restriction on the lengths, comes from the multiplier $x$.

Remark 3.2 Note that in absence of the feedback terms (with and without delay) this result can be see as an alternative proof via Lyapunov theory of Theorem 3.4 [1] (in our case with a more restrictive condition on the lengths).

\subsection{Observability approach}

In the previous section we obtained a stabilization result under the hypothesis that $L<\frac{\sqrt{3}}{2} \pi, \alpha>N / 2$ and for small initial data. Now we are going to prove a result without restrictions on the lengths that holds for $\alpha \geq N / 2$ and small initial data. The idea is to obtain an observability inequality in the linear system. The proof is based on a contradiction argument and hence we can not estimate the decay rate of the energy, contrary to Theorem 1.1 .

Theorem 3.1 Assume a, $b \in \mathbb{L}^{\infty}(\mathcal{T})$ componentwise non-negative that satisfy 1.1 and (1.2). Let $\left(\ell_{j}\right)_{j=1}^{N} \subset(0, \infty)$ and $T>\mathfrak{h}$, then there exists $C>0$ such that for all $U_{0}=\left(\underline{u}^{0}, \underline{z}^{0}(-\underline{h} \cdot, \cdot)\right) \in H$ we have the following observability inequality

$$
\begin{aligned}
& \sum_{j=1}^{N} \int_{0}^{\ell_{j}}\left(u_{j}^{0}(x)\right)^{2} d x+\sum_{j=1}^{N} h_{j} \int_{\omega_{j}} \int_{0}^{1} \xi_{j}(x)\left(z_{j}^{0}\left(-h_{j} \rho, x\right)\right)^{2} d x d \rho \\
\leq & C\left(\sum_{j=1}^{N} \int_{0}^{T}\left(\partial_{x} u_{j}(t, 0)\right)^{2} d t+\sum_{j=1}^{N} \int_{0}^{T} \int_{0}^{\ell_{j}} a_{j}(x)\left(u_{j}(t, x)\right)^{2} d x d t\right. \\
& \left.+\sum_{j=1}^{N} \int_{0}^{T} \int_{\omega_{j}}\left(z_{j}(t, 1, x)\right)^{2} d x d t+(2 \alpha-N) \int_{0}^{T}\left(u_{1}(t, 0)\right)^{2} d t\right),
\end{aligned}
$$

for $\left(\frac{u}{\underline{z}}\right)=S(\cdot) U_{0}$, solution of $(\underline{\mathrm{LKdVd}})$. 
Proof We follow the classical approach presented in [18].

Suppose that Obs is false. Then we can find a sequence $\left(U_{0}^{n}\right)_{n \in \mathbb{N}}=$ $\left(\underline{u}^{0, n}, \underline{z}^{0, n}(-\underline{h} \cdot, \cdot)\right)_{n \in \mathbb{N}} \subset H$ such that

$$
\sum_{j=1}^{N} \int_{0}^{\ell_{j}}\left(u_{j}^{0, n}(x)\right)^{2} d x+\sum_{j=1}^{N} h_{j} \int_{\omega_{j}} \int_{0}^{1} \xi_{j}(x)\left(z_{j}^{0, n}\left(-h_{j} \rho, x\right)\right)^{2} d x d \rho=1
$$

and for $\left(\underline{u}^{n}, \underline{z}^{n}\right)=S\left(\underline{u}^{0, n}, \underline{z}^{0, n}(-\underline{h} \cdot, \cdot)\right)$ we have

$$
\begin{aligned}
& \sum_{j=1}^{N} \int_{0}^{T}\left(\partial_{x} u_{j}^{n}(t, 0)\right)^{2} d t+\sum_{j=1}^{N} \int_{0}^{T} \int_{0}^{\ell_{j}} a_{j}(x)\left(u_{j}^{n}(t, x)\right)^{2} d x d t \\
+ & \sum_{j=1}^{N} \int_{0}^{T} \int_{\omega_{j}}\left(z_{j}^{n}(t, 1, z)\right)^{2} d x d t+(2 \alpha-N) \int_{0}^{T}\left(u_{1}^{n}(t, 0)\right)^{2} \rightarrow 0
\end{aligned}
$$

when $n \rightarrow \infty$. Now using 2.22 for $g=0$ we get that $\left(\underline{u}^{n}\right)_{n \in \mathbb{N}}$ is bounded in $L^{2}\left(0, T ; \mathbb{H}_{e}^{1}(\mathcal{T})\right)$ and then as $\partial_{t} u_{j}^{n}=-\partial_{x} u_{j}^{n}-\partial_{x}^{3} u_{j}^{n}-a_{j} u_{j}^{n}-\bar{b}_{j} z_{j}^{n}(1)$, we have that $\left(\partial_{t} u_{j}^{n}\right)_{n \in \mathbb{N}}$ is bounded in $L^{2}\left(0, T ; H^{-2}\left(0, \ell_{j}\right)\right)$. Using the Aubin-Lions Lemma, we can deduce that $\left(\underline{u}^{n}\right)_{n \in \mathbb{N}}$ is relatively compact in $L^{2}\left(0, T ; \mathbb{L}^{2}(\mathcal{T})\right)$ and hence we can assume that it is convergent in $L^{2}\left(0, T ; \mathbb{L}^{2}(\mathcal{T})\right)$.

Moreover for $T>\mathfrak{h}$ since $z_{j}^{n}(t, \rho, x)=\left.u_{j}^{n}\right|_{\omega_{j}}\left(t-h_{j} \rho, x\right)$ we have

$$
\begin{gathered}
\int_{\omega_{j}} \int_{0}^{1}\left(z_{j}^{n}(T, \rho, x)\right)^{2} d \rho d x=\int_{\omega_{j}} \int_{0}^{1}\left(u_{j}^{n}\left(T-\rho h_{j}, x\right)\right)^{2} d \rho d x \\
\leq \frac{1}{h_{j}} \int_{\omega_{j}} \int_{0}^{T}\left(u_{j}(t, x)\right)^{2} d t d x .
\end{gathered}
$$

Now thanks to 2.7

$$
\begin{gathered}
\left\|\underline{z^{0, n}}(-\underline{h} \cdot, \cdot)\right\|_{\mathbb{L}^{2}(\Omega)}^{2} \leq\left\|\underline{z}^{n}(T, \cdot, \cdot)\right\|_{\mathbb{L}^{2}(\Omega)}^{2}+\sum_{j=1}^{N} \frac{1}{h_{j}} \int_{0}^{T} \int_{\omega_{j}}\left|z_{j}^{n}(t, 1, x)\right|^{2} d x d t . \\
\leq \sum_{j=1}^{N} \frac{1}{h_{j}} \int_{0}^{T} \int_{\omega_{j}}\left(u_{j}^{n}(t, x)\right)^{2} d x d t+\sum_{j=1}^{N} \frac{1}{h_{j}} \int_{0}^{T} \int_{\omega_{j}}\left|z_{j}^{n}(t, 1, x)\right|^{2} d x d t
\end{gathered}
$$

and hence $\left(\underline{z}^{0, n}(-\underline{h} \cdot, \cdot)\right)_{n \in \mathbb{N}}$ is a Cauchy sequence in $\mathbb{L}^{2}(\Omega)$ using (3.2). Moreover using 2.6 and 3.2 we get that $\left(\underline{u}^{0, n}\right)_{n \in \mathbb{N}}$ is a Cauchy sequence in $\mathbb{L}^{2}(\mathcal{T})$.

Let $U_{0}=\left(\underline{u}^{0}, \underline{z}^{0}(-\underline{h} \cdot, \cdot)\right)=\lim _{n \rightarrow \infty}\left(\underline{u}^{0, n}, \underline{z}^{0, n}(-\underline{h} \cdot, \cdot)\right)$ in $H$ and $(\underline{u}, \underline{z})=$ $S(\cdot)\left(\underline{u}^{0}, \underline{z}^{0}(-\underline{h} \cdot, \cdot)\right)$. By Proposition 2.1 we have: 


$$
\begin{aligned}
& \sum_{j=1}^{N} \int_{0}^{T} \int_{0}^{\ell_{j}} a_{j}(x)\left(u_{j}^{n}(t, x)\right)^{2} d x d t+\sum_{j=1}^{N} \int_{0}^{T} \int_{\omega_{j}} b_{j}(x)\left(z_{j}^{n}(t, 1, x)\right)^{2} d x d t \\
& \underset{n \rightarrow \infty}{\longrightarrow} \sum_{j=1}^{N} \int_{0}^{T} \int_{0}^{\ell_{j}} a_{j}(x)\left(u_{j}(t, x)\right)^{2} d x d t+\sum_{j=1}^{N} \int_{0}^{T} \int_{\omega_{j}} b_{j}(x)\left(z_{j}(t, 1, x)\right)^{2} d x d t .
\end{aligned}
$$

Thus

$$
\sum_{j=1}^{N} \int_{0}^{\ell_{j}}\left(u_{j}^{0}(x)\right)^{2} d x+\sum_{j=1}^{N} h_{j} \int_{\omega_{j}} \int_{0}^{1} \xi_{j}(x)\left(z_{j}^{0}\left(-h_{j} \rho, x\right)\right)^{2} d x d \rho=1
$$

and

$$
\sum_{j=1}^{N} \int_{0}^{T} \int_{0}^{\ell_{j}} a_{j}(x)\left(u_{j}(t, x)\right)^{2} d x d t+\sum_{j=1}^{N} \int_{0}^{T} \int_{\omega_{j}} b_{j}(x)\left(z_{j}(t, 1, x)\right)^{2} d x d t=0 .
$$

As $z_{j}(t, 1, x)=u_{j}\left(t-h_{j}, x\right)=0$ in $(0, T) \times \omega_{j}$, we can deduce that $\underline{z}^{0}=0$ and $\underline{z}=0$. Moreover $u_{j}=0$ on $(0, T) \times \omega_{j}$, and as $u_{j}$ is solution of $\partial_{t} u_{j}+\partial_{x} u_{j}+\partial_{x}^{3} u_{j}=0$ thanks to Holmgrem's Theorem, $u_{j}=0$ in $(0, T) \times\left(0, \ell_{j}\right)$. Thus $(\underline{u}, \underline{z})=(\underline{0}, \underline{0})$ and we get a contradiction which ends the proof.

Remark 3.3 Note that in the case $\alpha=N / 2$ the term of $\left\|u_{1}(t, 0)\right\|_{L^{2}(0, T)}^{2}$ disappear of Obs.

Now from the observability inequality (Obs), we can obtain the exponential stability of the linear system ( $\mathrm{LKdVd}$.

Theorem 3.2 Assume $a, b \in \mathbb{L}^{\infty}(\mathcal{T})$ componentwise non-negative that satisfy (1.1) and 1.2 . Let $\left(\ell_{j}\right)_{j=1}^{N} \bar{\subset}(0, \infty)$, then for all $\left(\underline{u}^{0}, \underline{z}^{0}(-\underline{h} \cdot, \cdot)\right) \in H$, the energy of the system (LKdVd) defined by (1.6) decays exponentially, i.e, there exists $C>0$ and $\mu>0$ such that $E(t) \leq C E(0) e^{-\mu t}$ for all $t>0$.

Proof We follow [21, 10] (see also [17]). Note that for $U_{0} \in D(\mathcal{A})$ the energy of (LKdVd) satisfies for $C_{1}>0$

$$
\begin{gathered}
\frac{d}{d t} E(t) \leq-C_{1}\left((2 \alpha-N) \int_{0}^{T}\left|u_{1}(t, 0)\right|^{2} d t+\sum_{j=1}^{N} \int_{0}^{T}\left|\partial_{x} u_{j}(t, 0)\right|^{2}\right. \\
\left.+\sum_{j=1}^{N} \int_{0}^{\ell_{j}} a_{j}(x)\left|u_{j}(t, x)\right|^{2} d x+\sum_{j=1}^{N} \int_{\omega_{j}}\left|u_{j}\left(t-h_{j}, x\right)\right|^{2} d x\right) .
\end{gathered}
$$

Integrating between 0 and $T>\mathfrak{h}$ we have 


$$
\begin{aligned}
& E(T)-E(0) \leq-C_{1}\left((2 \alpha-N) \int_{0}^{T}\left|u_{1}(t, 0)\right|^{2} d t+\sum_{j=1}^{N} \int_{0}^{T}\left|\partial_{x} u_{j}(t, 0)\right|^{2} d t\right. \\
& \left.+\sum_{j=1}^{N} \int_{0}^{T} \int_{0}^{\ell_{j}} a_{j}(x)\left|u_{j}(t, x)\right|^{2} d x d t+\sum_{j=1}^{N} \int_{\omega_{j}} \int_{0}^{T}\left|u_{j}\left(t-h_{j}, x\right)\right|^{2} d x d t\right) .
\end{aligned}
$$

The last expression can be rewritten as

$$
\begin{gathered}
(2 \alpha-N) \int_{0}^{T}\left|u_{1}(t, 0)\right|^{2} d t+\sum_{j=1}^{N} \int_{0}^{T} \int_{0}^{\ell_{j}} a_{j}(x)\left|u_{j}(t, x)\right|^{2} d x d t \\
+\sum_{j=1}^{N} \int_{0}^{T}\left|\partial_{x} u_{j}(t, 0)\right|^{2} d t+\sum_{j=1}^{N} \int_{\omega_{j}} \int_{0}^{T}\left|u_{j}\left(t-h_{j}, x\right)\right|^{2} d x d t \leq \frac{1}{C_{1}}(E(0)-E(T)) .
\end{gathered}
$$

Using that the energy is non-increasing and (Obs) we get

$$
\begin{aligned}
& E(T) \leq E(0) \leq C\left((2 \alpha-N) \int_{0}^{T}\left|u_{1}(t, 0)\right|^{2} d t+\sum_{j=1}^{N} \int_{0}^{T} \int_{0}^{\ell_{j}} a_{j}(x)\left|u_{j}(t, x)\right|^{2} d x d t\right. \\
& \left.+\sum_{j=1}^{N} \int_{0}^{T}\left|\partial_{x} u_{j}(t, 0)\right|^{2} d t+\sum_{j=1}^{N} \int_{\omega_{j}} \int_{0}^{T}\left|u_{j}\left(t-h_{j}, x\right)\right|^{2} d x d t\right) \leq \frac{C}{C_{1}}(E(0)-E(T))
\end{aligned}
$$

which implies

$$
E(T) \leq \gamma E(0), \text { with } \gamma=\frac{\frac{C}{C_{1}}}{1+\frac{C}{C_{1}}}<1 .
$$

Now as the system is invariant in time, we can repeat this argument on $[(m-$ 1) $T, m T]$ for $m=1,2, \cdots$ to obtain

$$
E(m T) \leq \gamma E((m-1) T) \leq \cdots \leq \gamma^{m} E(0) .
$$

Hence we have $E(m T) \leq e^{-\mu m T} E(0)$ where $\mu=\frac{1}{T} \ln \left(\frac{1}{\gamma}\right)>0$. Let $t>\mathfrak{h}$. Then there exists $m \in \mathbb{N}^{*}$ such that $(m-1) T<t \leq m T$, and then using again the non-increasing property of the energy we get

$$
E(t) \leq E((m-1) T) \leq e^{-\mu(m-1) T} E(0) \leq \frac{1}{\gamma} e^{-\mu t} E(0) .
$$

By density of $D(\mathcal{A})$ in $H$ we can extend our result to any initial data in $H$.

Remark 3.4 Note that if $\alpha=N / 2$ the term of $\left\|u_{1}(t, 0)\right\|_{L^{2}(0, T)}^{2}$ disappears of 3.3. which is consistent with Remark 3.3

To end this part we give the proof of Theorem 1.2 , inspired by [21, 3, 9]. 
Proof of Theorem 1.2:

Let $\left(\underline{u}^{0}, \underline{z}^{0}(-\underline{h} \cdot, \cdot)\right) \in H$ with $\left\|\left(\underline{u}^{0}, \underline{z}^{0}(-\underline{h} \cdot, \cdot)\right)\right\|_{H} \leq r$ for some $r>0$ that will be chosen after, then the solution $\underline{u}$ of $(\overline{\mathrm{KdVd}}$ can be decomposed into $\underline{u}=\underline{\bar{u}}+\underline{\tilde{u}}$ respectively solutions of

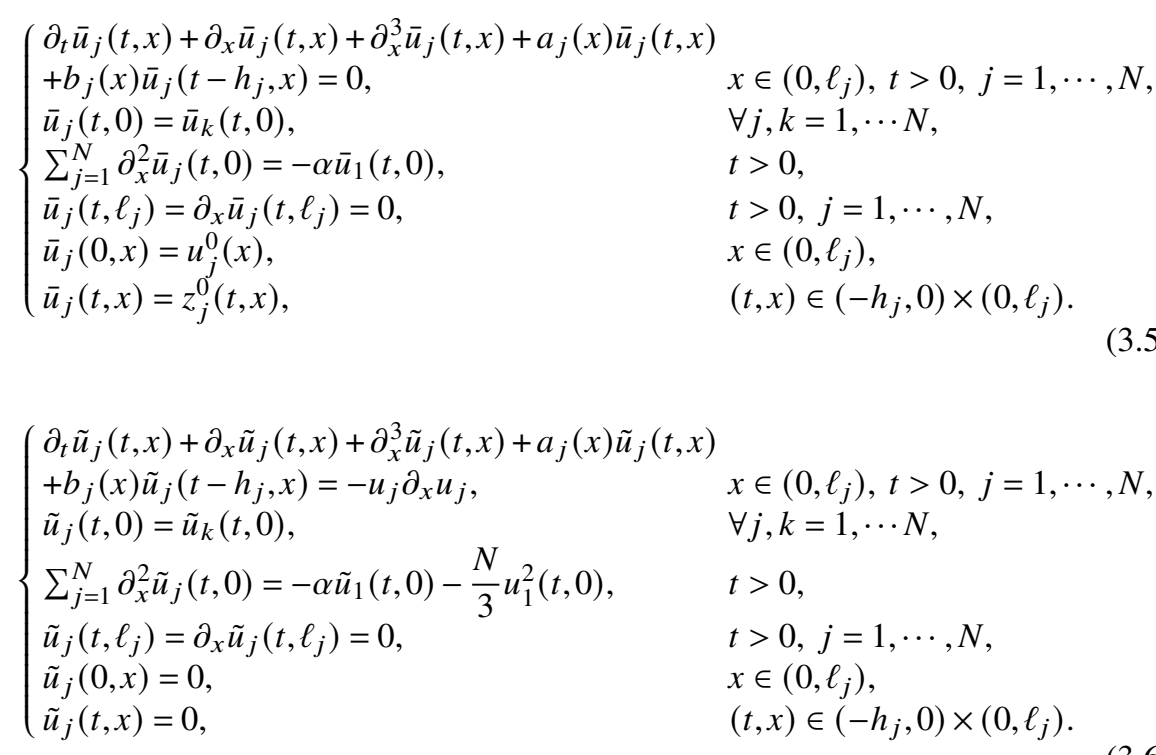

In simple words $\bar{u}$ is solution of (LKdVd) with initial data $\left(u^{0}, z^{0}(-h \cdot, \cdot)\right)$ and $\tilde{u}$ is solution of (2.28) with null initial data and source terms $f_{j}=u_{j} \partial_{x} u_{j}$ and $g=$ $-\frac{N}{3} u_{1}^{2}(t, 0)$. Then using Proposition 2.5. Proposition 2.6 and Theorem 3.2. we have

$$
\begin{gathered}
\|\underline{u}(T), \underline{z}(T)\|_{H} \leq\|\underline{\tilde{u}}(T), \underline{\tilde{z}}(T)\|_{H}+\|\underline{\tilde{u}}(T), \underline{z}(T)\|_{H} \\
\leq C\left(\left\|\underline{u} \partial_{x} \underline{u}\right\|_{L^{1}\left(0, T ; \mathbb{L}^{2}(\mathcal{T})\right)}+\left\|u_{1}^{2}(t, 0)\right\|_{L^{2}(0, T)}\right)+\gamma\left\|U_{0}\right\|_{H} \leq \gamma\left\|U_{0}\right\|_{H}+C\|\underline{u}\|_{\mathbb{B}}^{2},
\end{gathered}
$$

where $\gamma<1$. Our plan now is to deal with the term $\|\underline{u}\|_{\mathbb{B}}^{2}$. Multiplying $\left(\mathrm{KdVd}\right.$ by $u_{j}$ and integrating in $(0, s) \times\left(0, \ell_{j}\right)$ we can get

$$
\begin{aligned}
& \|\underline{u}(s, \cdot)\|_{\mathbb{L}^{2}(\mathcal{T})}^{2}+\sum_{j=1}^{N} \int_{0}^{s}\left|\partial_{x} u_{j}(t, 0)\right|^{2} d s+(2 \alpha-N) \int_{0}^{s}\left|u_{1}(t, 0)\right|^{2} d s \\
& +2 \sum_{j=1}^{N} \int_{0}^{s} \int_{0}^{\ell_{j}}\left(a_{j}-b_{j}\right)\left|u_{j}\right|^{2} d x d s \leq\left\|\underline{u}^{0}\right\|_{\mathbb{L}^{2}(\mathcal{T})}^{2}+\left\|\underline{z}^{0}(-\underline{h}, \cdot)\right\|_{\mathbb{L}^{2}(\Omega)}^{2}
\end{aligned}
$$

Now multiplying $\overline{\mathrm{KdVd}}$ by $q_{j} u_{j}$ with $q_{j}=\frac{x\left(2 \ell_{j}-x\right)}{\ell_{j}^{2}}$ we can obtain

$$
\left\|u_{1}(t, 0)\right\|_{L^{2}(0, T)}^{2} \leq C\left\|U_{0}\right\|_{H}^{2}+\frac{2}{3} \sum_{j=1}^{N} \int_{0}^{T} \int_{0}^{\ell_{j}} u_{j}^{3}(t, x) d x d t
$$


As $\forall j=1, \cdots, N u_{j} \in L^{2}\left(0, T ; H^{1}\left(0, \ell_{j}\right)\right)$ and $H^{1}\left(0, \ell_{j}\right)$ embeds into $C\left(\left[0, \ell_{j}\right]\right)$ we get following [3, 17]

$$
\sum_{j=1}^{N} \int_{0}^{T} \int_{0}^{\ell_{j}}\left|u_{j}\right|^{3} d x d t \leq C T^{1 / 2}\left\|U_{0}\right\|_{H}^{2}\|\underline{u}\|_{L^{2}\left(0, T ; \mathbb{H}_{e}^{1}(\mathcal{T})\right)}
$$

and then

$$
\left\|u_{1}(t, 0)\right\|_{L^{2}(0, T)}^{2} \leq C\left\|U_{0}\right\|_{H}^{2}+C T^{1 / 2}\left\|U_{0}\right\|_{H}^{2}\|\underline{u}\|_{L^{2}\left(0, T ; \mathbb{H}_{e}^{1}(\mathcal{T})\right)} .
$$

On a similar way multiplying $(\mathrm{KdVd})$ by $x u_{j}$ and using the last inequality we deduce

$$
\left\|\partial_{x} \underline{u}\right\|_{L^{2}\left(0, T ; \mathbb{L}^{2}(\mathcal{T})\right)}^{2} \leq C\left(\left\|U_{0}\right\|_{H}^{2}+\left\|U_{0}\right\|_{H}^{2}\|\underline{u}\|_{L^{2}\left(0, T ; \mathbb{H}_{e}^{1}(\mathcal{T})\right)}\right) .
$$

Using Young's inequality, we can find $C>0$ such that

$$
\left\|\partial_{x} \underline{u}\right\|_{L^{2}\left(0, T ; \mathbb{L}^{2}(\mathcal{T})\right)}^{2} \leq C\left(\left\|U_{0}\right\|_{H}^{2}+\left\|U_{0}\right\|_{H}^{4}\right) .
$$

Combining the estimates 3.7$)$ and $(3.8)$ we get

$$
\|\underline{u}(T), \underline{z}(T)\|_{H} \leq\left\|U_{0}\right\|_{H}\left(\gamma+C\left\|U_{0}\right\|_{H}+C\left\|U_{0}\right\|_{H}^{3}\right) .
$$

Taking $\left\|U_{0}\right\|_{H} \leq \epsilon$ for $\epsilon$ small enough such that $\gamma+C \epsilon+C \epsilon^{3}<1$, then the proof follows in the same way as Theorem 3.2 .

\subsection{Semi-global Stabilization}

The aim of this section is to prove Theorem 1.3 that is a semi-global result without restriction on the lengths and for $\alpha \geq N / 2$. The main idea is to obtain an observability inequality as $\mathrm{Obs}$ working directly with the nonlinear system $\mathrm{KdVd}$. In this context two main difficulties appears, the first one is to pass to the limit in the nonlinear term and the second that Holmgrem's Theorem does not apply in the nonlinear case.

Proof of Theorem 1.3 :

To prove this result we adapt the techniques of[3]. We need the next Unique Continuation Property of Saut and Scheurer.

Theorem 3.3 (Theorem 4.2, [20]) Let $L>0$ and $y \in L^{2}\left(0, T ; H^{3}(0, L)\right)$ be a solution of

$$
y_{t}+y_{x}+y_{x x x}+y y_{x}=0,
$$

such that $y(t, x)=0$, for $(t, x) \in\left(t_{1}, t_{2}\right) \times \omega$, where $\omega$ is a nonempty open subset of $(0, L)$. Then $y(t, x)=0$, for $(t, x) \in\left(t_{1}, t_{2}\right) \times(0, L)$. 
First defining $z_{j}(t, \rho, x)=\left.u_{j}\right|_{\omega_{j}}\left(t-h_{j} \rho, x\right) x \in \omega_{j}, \rho \in(0,1)$, for $\underline{u}$ solution of (KdVd, we can check that

$$
\begin{cases}h_{j} \partial_{t} z_{j}(t, \rho, x)+\partial_{\rho} z_{j}(t, \rho, x)=0, & x \in \omega_{j}, \rho \in(0,1), t>0 \\ z_{j}(t, 0, x)=u_{j}(t, x), & x \in \omega_{j}, t>0 \\ z_{j}(0, \rho, x)=\left.u_{j}\right|_{\omega_{j}}\left(-h_{j} \rho, x\right)=z_{j}^{0}\left(-h_{j} \rho, x\right), \rho \in(0,1)\end{cases}
$$

Multiplying $(3.10)$ by $z_{j}$ and integrating on $(0, s) \times(0,1) \times \omega_{j}$ we can obtain

$$
\left\|\underline{z}^{0}(-\underline{h} \cdot, \cdot)\right\|_{\mathbb{L}^{2}(\Omega)}^{2} \leq\|\underline{z}(s, \cdot, \cdot)\|_{\mathbb{L}^{2}(\Omega)}^{2}+\sum_{j=1}^{N} \frac{1}{h_{j}} \int_{0}^{T} \int_{\omega_{j}}\left|z_{j}(t, 1, x)\right|^{2} d x d t .
$$

Now integrating this relation on $(0, T)$

$$
\begin{gathered}
T\left\|\underline{z}^{0}(-\underline{h}, \cdot)\right\|_{\mathbb{L}^{2}(\Omega)}^{2} \leq \sum_{j=1}^{N} \int_{0}^{T} \int_{0}^{1} \int_{\omega_{j}}\left|z_{j}(t, \rho, x)\right|^{2} d x d \rho d t \\
+\sum_{j=1}^{N} \frac{T}{h_{j}} \int_{0}^{T} \int_{\omega_{j}}\left|z_{j}(t, 1, x)\right|^{2} d x d t .
\end{gathered}
$$

Note now that for $T>\mathfrak{h}$ we have

$$
\begin{gathered}
\int_{0}^{T} \int_{0}^{1} \int_{\omega_{j}}\left|z_{j}(t, \rho, x)\right|^{2} d x d \rho d t=\int_{0}^{T} \int_{0}^{1} \int_{\omega_{j}}\left|u_{j}\left(t-\rho h_{j}, x\right)\right|^{2} d x d \rho d t \\
=\int_{0}^{T} \int_{t-h_{j}}^{t} \int_{\omega_{j}}\left|u_{j}(s, x)\right|^{2} d x d s d t \leq \frac{T}{h_{j}} \int_{-h_{j}}^{T} \int_{\omega_{j}}\left|u_{j}(s, x)\right|^{2} d x d s \\
=\frac{T-h_{j}}{h_{j}} \int_{-h_{j}}^{T} \int_{\omega_{j}}\left|u_{j}(s, x)\right|^{2} d x d s+\frac{T}{h_{j}} \int_{T-h_{j}}^{T} \int_{\omega_{j}}\left|u_{j}(s, x)\right|^{2} d x d s \\
\leq \frac{T}{h_{j}} \int_{0}^{T} \int_{\omega_{j}}\left(\left|u_{j}(t, x)\right|^{2}+\left|u_{j}\left(t-h_{j}, x\right)\right|^{2}\right) d x d t \\
\leq C\left(\int_{0}^{T} \int_{\omega_{j}} a_{j}\left|u_{j}\right|^{2} d x d t+\int_{0}^{T} \int_{\omega_{j}}\left|z_{j}(t, 1, x)\right|^{2} d x d t\right)
\end{gathered}
$$

which gives us

$$
T\left\|\underline{z}^{0}(-\underline{h} \cdot, \cdot)\right\|_{\mathbb{L}^{2}(\Omega)}^{2} \leq C\left(\sum_{j=1}^{N} \int_{0}^{T} \int_{\omega_{j}} a_{j}\left|u_{j}\right|^{2} d x d t+\int_{0}^{T} \int_{\omega_{j}}\left|z_{j}(t, 1, x)\right|^{2} d x d t\right) .
$$


Multiplying (KdVd) by $u_{j}$ and integrating on time and space, we have

$$
\begin{gathered}
\|\underline{u}(s, \cdot)\|_{\mathbb{L}^{2}(\mathcal{T})}^{2}+(2 \alpha-N) \int_{0}^{s}\left|u_{1}(t, 0)\right|^{2} d t+2 \sum_{j=1}^{N} \int_{0}^{s} \int_{0}^{\ell_{j}} a_{j}\left|u_{j}\right|^{2} d x d t \\
+\sum_{j=1}^{N} \int_{0}^{s}\left|\partial_{x} u_{j}(t, 0)\right|^{2} d t+2 \sum_{j=1}^{N} \int_{0}^{s} \int_{0}^{\ell_{j}} b_{j} u_{j}\left(t-h_{j}, x\right) u_{j}(t, x) d x d t=\left\|\underline{u}^{0}\right\|_{\mathbb{L}^{2}(\mathcal{T})}^{2} .
\end{gathered}
$$

Integrating again over $(0, T)$ this relation, we get,

$$
\begin{gathered}
T\left\|\underline{u}^{0}\right\|_{\mathbb{L}^{2}(\mathcal{T})}^{2} \leq \int_{0}^{T}\|\underline{u}(t, \cdot)\|_{\mathbb{L}^{2}(\mathcal{T})}^{2} d t+T \int_{0}^{T} \sum_{j=1}^{N}\left|\partial_{x} u_{j}(t, 0)\right|^{2} d t \\
+(2 \alpha-N) T \int_{0}^{T}\left|u_{1}(t, 0)\right|^{2} d t+2 T \sum_{j=1}^{N} \int_{0}^{T} \int_{0}^{\ell_{j}} a_{j}\left|u_{j}\right|^{2} d x d t \\
+2 T \sum_{j=1}^{N} \int_{0}^{T} \int_{0}^{\ell_{j}} b_{j} u_{j}\left(t-h_{j}, x\right) u_{j}(t, x) d x d t .
\end{gathered}
$$

Note now that

$$
\begin{gathered}
\int_{0}^{T} \int_{0}^{\ell_{j}} b_{j} u_{j}\left(t-h_{j}, x\right) u_{j}(t, x) d x d t \\
\leq \frac{1}{2} \int_{0}^{T} \int_{\omega_{j}} b_{j}\left|u_{j}\right|^{2} d x d t+\frac{1}{2} \int_{0}^{T} \int_{\omega_{j}} b_{j}\left|u_{j}\left(t-h_{j}, x\right)\right|^{2} d x d t,
\end{gathered}
$$

and then

$$
\begin{gathered}
T\left\|\underline{u}^{0}\right\|_{\mathbb{L}^{2}(\mathcal{T})}^{2} \leq C\left(\int_{0}^{T}\|\underline{u}(t, \cdot)\|_{\mathbb{L}^{2}(\mathcal{T})}^{2} d t+(2 \alpha-N) \int_{0}^{T}\left|u_{1}(t, 0)\right|^{2} d t\right. \\
+\sum_{j=1}^{N} \int_{0}^{T}\left|\partial_{x} u_{j}(t, 0)\right|^{2} d t+\sum_{j=1}^{N} \int_{0}^{T} \int_{0}^{\ell_{j}} a_{j}\left|u_{j}\right|^{2} d x d t \\
\left.+\sum_{j=1}^{N} \int_{0}^{T} \int_{\omega_{j}}\left|z_{j}(t, 1, x)\right|^{2} d x d t\right) .
\end{gathered}
$$

Joining the estimates for $\underline{u}^{0}$ and $\underline{z}^{0}$ we get

$$
\begin{gathered}
\sum_{j=1}^{N} \int_{0}^{\ell_{j}}\left|u_{j}^{0}\right|^{2} d x+\sum_{j=1}^{N} h_{j} \int_{\omega_{j}} \int_{0}^{1} \xi_{j}(x)\left|z_{j}^{0}\left(-h_{j}, \rho, x\right)\right|^{2} d x d \rho \\
\leq C\left(\int_{0}^{T}\|\underline{u}(t, \cdot)\|_{\mathbb{L}^{2}(\mathcal{T})}^{2} d t+\sum_{j=1}^{N} \int_{0}^{T}\left|\partial_{x} u_{j}(t, 0)\right|^{2} d t+(2 \alpha-N) \int_{0}^{T}\left|u_{1}(t, 0)\right|^{2} d t\right. \\
\left.+\sum_{j=1}^{N} \int_{0}^{T} \int_{0}^{\ell_{j}} a_{j}\left|u_{j}\right|^{2} d x d t+\sum_{j=1}^{N} \int_{0}^{T} \int_{\omega_{j}}\left|z_{j}(t, 1, x)\right|^{2} d x d t\right) .
\end{gathered}
$$


This inequality is quite similar to the observability inequality (Obs). Moreover to prove our result it is enough to get that for any $T, R>0$ there exists $C=C(R, T)>0$ such that for any solutions of (KdVd) with $\left\|U_{0}\right\|_{H} \leq R$ we have

$$
\begin{gathered}
\int_{0}^{T}\|\underline{u}(t, \cdot)\|_{\mathbb{L}^{2}(\mathcal{T})}^{2} d t \leq C\left(\sum_{j=1}^{N} \int_{0}^{T}\left|\partial_{x} u_{j}(t, 0)\right|^{2} d t+(2 \alpha-N) \int_{0}^{T}\left|u_{1}(t, 0)\right|^{2} d t\right. \\
\left.+\sum_{j=1}^{N} \int_{0}^{T} \int_{0}^{\ell_{j}} a_{j}\left|u_{j}\right|^{2} d x d t+\sum_{j=1}^{N} \int_{0}^{T} \int_{\omega_{j}}\left|z_{j}(t, 1, x)\right|^{2} d x d t\right) .
\end{gathered}
$$

Suppose that this inequality does not hold. Then there exists $\left(\underline{u}^{n}\right)_{n \in \mathbb{N}} \subset \mathbb{B}$ solution of (KdVd) with $\left\|U_{0}^{n}\right\|_{H} \leq R$ such that

$$
\lim _{n \rightarrow \infty} \frac{\int_{0}^{T}\left\|\underline{u}^{n}(t, \cdot)\right\|_{L^{2}(\mathcal{T})}^{2} d t}{\left\|\partial_{x} \underline{u}^{n}(t, 0)\right\|_{L^{2}(0, T)}^{2}+(2 \alpha-N)\left\|u_{1}^{n}(t, 0)\right\|_{L^{2}(0, T)}^{2}+\sum_{j=1}^{N} \int_{0}^{T} \int_{0}^{\ell_{j}} a_{j}\left|u_{j}^{n}\right|^{2} d x d t+\sum_{j=1}^{N} \int_{0}^{T} \int_{\omega_{j}}\left|z_{j}^{n}(t, 1, x)\right|^{2} d x d t}=\infty .
$$

Take $\lambda^{n}=\left\|\underline{u}^{n}\right\|_{L^{2}\left(0, T ; \mathbb{L}^{2}(\mathcal{T})\right)}, \underline{v}^{n}:=\frac{u^{n}}{\lambda^{n}}$ and $y_{j}^{n}(t, \rho, x)=\left.v_{j}^{n}\right|_{\omega_{j}}\left(t-h_{j} \rho, x\right) x \in \omega_{j}$, $\rho \in(0,1)$. Then, $\underline{v}^{n}$ satisfies

$$
\begin{cases}\partial_{t} v_{j}^{n}(t, x)+\partial_{x} v_{j}^{n}(t, x)+\partial_{x}^{3} v_{j}^{n}(t, x)+a_{j}(x) v_{j}^{n}(t, x) & \\ +b_{j}(x) v_{j}^{n}\left(t-h_{j}, x\right)+\lambda^{n} v_{j}^{n}(t, x) \partial_{x} v_{j}^{n}(t, x)=0, & x \in\left(0, \ell_{j}\right), t>0, j=1, \cdots, N, \\ v_{j}^{n}(t, 0)=v_{k}^{n}(t, 0), & \forall j, k=1, \cdots N, \\ \sum_{j=1}^{N} \partial_{x}^{2} v_{j}^{n}(t, 0)=-\alpha v_{1}^{n}(t, 0)-\lambda^{n} \frac{N}{3}\left(v_{1}^{n}(t, 0)\right)^{2}, & t>0, \\ v_{j}^{n}\left(t, \ell_{j}\right)=\partial_{x} v_{j}^{n}\left(t, \ell_{j}\right)=0, & t>0, j=1, \cdots, N, \\ \left\|\underline{v}^{n}\right\|_{L^{2}\left(0, T ; \mathbb{L}^{2}(\mathcal{T})\right)}=1 . & \end{cases}
$$

and

$$
\begin{gathered}
\left\|\partial_{x} \underline{v}^{n}(t, 0)\right\|_{L^{2}(0, T)}^{2}+(2 \alpha-N)\left\|v_{1}^{n}(t, 0)\right\|_{L^{2}(0, T)}^{2}+\sum_{j=1}^{N} \int_{0}^{T} \int_{0}^{\ell_{j}} a_{j}\left|v_{j}^{n}\right|^{2} d x d t \\
+\sum_{j=1}^{N} \int_{0}^{T} \int_{\omega_{j}}\left|y_{j}^{n}(t, 1, x)\right|^{2} d x d t \rightarrow 0 .
\end{gathered}
$$

Now multiplying 3.11 by $v_{j}^{n}$ and integrating over $(0, T) \times(0, t) \times\left(0, \ell_{j}\right)$ we can get

$$
\begin{gathered}
T\left\|\underline{v}^{n}(0, \cdot)\right\|_{\mathbb{L}^{2}(\mathcal{T})}^{2} \leq C\left(\int_{0}^{T}\left\|\underline{v}^{n}(t, \cdot)\right\|_{\mathbb{L}^{2}(\mathcal{T})}^{2} d t+\left\|\partial_{x} \underline{v}^{n}(t, 0)\right\|_{L^{2}(0, T)}^{2}+\left\|\underline{v}^{n}(-\underline{h} \cdot, \cdot)\right\|_{\mathbb{L}^{2}(\Omega)}^{2}\right. \\
\left.+(2 \alpha-N)\left\|v_{1}^{n}(t, 0)\right\|_{L^{2}(0, T)}^{2}\right) .
\end{gathered}
$$

Now for $T>\mathfrak{h}$, 


$$
\begin{gathered}
\left\|\underline{v}^{n}(-\underline{h}, \cdot)\right\|_{\mathbb{L}^{2}(\Omega)}^{2}=\sum_{j=1}^{N} \int_{\omega_{j}} \int_{0}^{1}\left|v_{j}^{n}\left(-h_{j} \rho, x\right)\right|^{2} d \rho d x=\sum_{j=1}^{N} \frac{1}{h_{j}} \int_{\omega_{j}} \int_{-h_{j}}^{0}\left|v_{j}^{n}(t, x)\right|^{2} d t d x \\
\leq \sum_{j=1}^{N} \frac{1}{h_{j}} \int_{\omega_{j}} \int_{-h_{j}}^{T-h_{j}}\left|v_{j}^{n}(t, x)\right|^{2} d t d x=\sum_{j=1}^{N} \frac{1}{h_{j}} \int_{\omega_{j}} \int_{0}^{T}\left|v_{j}^{n}\left(t-h_{j}, x\right)\right|^{2} d t d x \\
=\sum_{j=1}^{N} \frac{1}{h_{j}} \int_{\omega_{j}} \int_{0}^{T}\left|y_{j}^{n}(t, 1, x)\right|^{2} d t d x .
\end{gathered}
$$

These estimates show us that $\left(\underline{v}^{n}(0, \cdot)\right)_{n \in \mathbb{N}}$ is bounded in $\mathbb{L}^{2}(\mathcal{T})$, also we can see that from the well-posedness of (KdVd) we get

$$
\lambda^{n}=\left\|\underline{u}^{n}\right\|_{L^{2}\left(0, T ; \mathbb{L}^{2}(\mathcal{T})\right)} \leq T\left\|U_{0}^{n}\right\|_{H} \leq T R .
$$

Consequently in the same sense as 3.8 we can obtain

$$
\left\|\underline{\underline{n}}^{n}\right\|_{L^{2}\left(0, T ; \mathbb{H} \mathbb{1}_{e}^{1}(\mathcal{T})\right)}^{2} \leq C\left(\left\|U_{0}^{n}\right\|_{H}^{2}+\left\|U_{0}^{n}\right\|_{H}^{4}\right) .
$$

Thus $\left(\underline{v}^{n}\right)_{n \in \mathbb{N}} \subset L^{2}\left(0, T ; \mathbb{H}_{e}^{1}(\mathcal{T})\right)$ is bounded and

$$
\left\|v_{j}^{n} \partial_{x} v_{j}^{n}\right\|_{L^{2}\left(0, T ; L^{1}\left(0, \ell_{j}\right)\right)} \leq\left\|\underline{v}^{n}\right\|_{C\left([0, T], \mathbb{L}^{2}(\mathcal{T})\right)}\left\|\underline{v}^{n}\right\|_{L^{2}\left(0, T ; ; \mathbb{H}_{e}^{1}(\mathcal{T})\right)},
$$

what implies that $\left(v_{j}^{n} \partial_{x} v_{j}^{n}\right)_{n \in \mathbb{N}}$ is subset of $L^{2}\left(0, T ; L^{1}\left(0, \ell_{j}\right)\right)$.

With this we can see that $\partial_{t} v_{j}^{n}=-\left(\partial_{x}^{3} v_{j}^{n}+\partial_{x} v_{j}^{n}+\lambda^{n} v_{j}^{n} \partial_{x} v_{j}^{n}+a_{j} v_{j}^{n}+b_{j} v_{j}^{n}\left(t-h_{j}\right)\right)$ is bounded in $L^{2}\left(0, T ; H^{-2}\left(0, \ell_{j}\right)\right)$ and hence by Aubin-Lions Lemma we can deduce that $\left(\underline{v}^{n}\right)_{n \in \mathbb{N}}$ is relatively compact $L^{2}\left(0, T ; \mathbb{L}^{2}(\mathcal{T})\right)$ and hence we can assume that $\underline{v}^{n}$ converges strongly at $\underline{v}$ in $L^{2}\left(0, T ; \mathbb{L}^{2}(\mathcal{T})\right)$ with $\|\underline{v}\|_{L^{2}\left(0, T ; \mathbb{L}^{2}(\mathcal{T})\right)}=1$. Furthermore, passing to the limit on 3.12 we get

$$
\begin{gathered}
\left\|\partial_{x} \underline{\underline{v}}(t, 0)\right\|_{L^{2}(0, T)}^{2}+(2 \alpha-N)\left\|v_{1}(t, 0)\right\|_{L^{2}(0, T)}^{2}+\sum_{j=1}^{N} \int_{0}^{T} \int_{\omega_{j}}\left|v_{j}\left(t-h_{j}\right)\right|^{2} d x d t \\
+\sum_{j=1}^{N} \int_{0}^{T} \int_{0}^{\ell_{j}} a_{j}\left|v_{j}\right|^{2} d x d t \leq \liminf \left(\left\|\partial_{x} \underline{v}^{n}(t, 0)\right\|_{L^{2}(0, T)}^{2}+(2 \alpha-N)\left\|v_{1}^{n}(t, 0)\right\|_{L^{2}(0, T)}^{2}\right. \\
\left.+\sum_{j=1}^{N} \int_{0}^{T} \int_{0}^{\ell_{j}} a_{j}\left|v_{j}^{n}\right|^{2} d x d t+\sum_{j=1}^{N} \int_{0}^{T} \int_{\omega_{j}}\left|v_{j}^{n}\left(t-h_{j}\right)\right|^{2} d x d t\right)
\end{gathered}
$$

Thus $v_{j}(t, x) \equiv 0$ in $\left(-h_{j}, T\right) \times \omega_{j}$ and $(2 \alpha-N) v_{j}(t, 0)=\partial_{x} v_{j}(t, 0)=0$ in $(0, T)$ for all $j=1, \cdots, N$. Also as $\left(\lambda^{n}\right)_{n \in \mathbb{N}}$ is bounded, we can extract a convergent subsequence such that $\lambda^{n} \rightarrow \lambda \geq 0$, consequently $\underline{v}$ satisfies $\|\underline{v}\|_{L^{2}\left(0, T ; \mathbb{L}^{2}(\mathcal{T})\right)}=1$ and the following equation 


$$
\begin{cases}\partial_{t} v_{j}+\partial_{x} v_{j}+\partial_{x}^{3} v_{j}+\lambda v_{j} \partial_{x} v_{j}=0, & \forall x \in\left(0, \ell_{j}\right), t>0, j=1, \cdots, N, \\ (2 \alpha-N) v_{j}(t, 0)=\partial_{x} v_{j}(t, 0)=0, & \forall j=1, \cdots N, \\ v_{j}\left(t, \ell_{j}\right)=\partial_{x} v_{j}\left(t, \ell_{j}\right)=0, & \forall j=1, \cdots N, \\ \sum_{j=1}^{N} \partial_{x}^{2} v_{j}(t, 0)=-\alpha v_{1}(t, 0)-\lambda \frac{N}{3}\left(v_{1}(t, 0)\right)^{2}, & t>0, \\ v_{j}(t, x)=0 & (t, x) \in\left(-h_{j}, T\right) \times \omega_{j} .\end{cases}
$$

1. If $\lambda=0$ the system satisfied by $\underline{v}$ is linear, then thanks Holmgrem's Theorem $\underline{v}=0$, that contradicts the fact that $\|\underline{v}\|_{L^{2}\left(0, T ; \mathbb{L}^{2}(\mathcal{T})\right)}=1$.

2. If $\lambda>0$. In this case we have to prove that $v_{j} \in L^{2}\left(0, T ; H^{3}\left(0, \ell_{j}\right)\right)$ in order to apply Theorem 3.3 Consider $w_{j}=\partial_{t} v_{t}$ then

$$
\begin{cases}\partial_{t} w_{j}+\partial_{x} w_{j}+\partial_{x}^{3} w_{j}+\lambda w_{j} \partial_{x} v_{j}+\lambda v_{j} \partial_{x} w_{j}=0, & x \in\left(0, \ell_{j}\right), t>0, j=1, \cdots, N, \\ (2 \alpha-N) w_{j}(t, 0)=\partial_{x} w_{j}(t, 0)=0, & \forall j=1, \cdots N, \\ w_{j}\left(t, \ell_{j}\right)=\partial_{x} w_{j}\left(t, \ell_{j}\right)=0, & \forall j=1, \cdots N, \\ \sum_{j=1}^{N} \partial_{x}^{2} w_{j}(t, 0)=-\alpha w_{1}(t, 0)-\lambda \frac{2 N}{3} w_{1}(t, 0) v_{1}(t, 0), & t>0, \\ w_{j}(t, x)=0 & (t, x) \in\left(-h_{j}, T\right) \times \omega_{j}, \\ w_{j}(0, x)=-v^{\prime}(0, x)-v^{\prime \prime \prime}(0, x)-\lambda v(0, x) v^{\prime}(0, x), & x \in\left(0, \ell_{j}\right), j=1, \cdots, N .\end{cases}
$$

Note that $w_{j}(0, x) \in H^{-3}\left(0, \ell_{j}\right)$, with Lemma A.2 [3] we can get that $w_{j}(0, x) \in$ $L^{2}\left(0, \ell_{j}\right)$ and $w_{j} \in C\left([0, T], L^{2}\left(0, \ell_{l}\right)\right) \cap L^{2}\left(0, T ; H^{1}\left(0, \ell_{j}\right)\right)$. Thus $\partial_{x}^{3} v_{j}=-\left(\partial_{t} v_{j}-\right.$ $\partial_{x} v_{j}-\lambda v_{j} \partial_{x} v_{j} \in L^{2}\left(0, T ; L^{2}(0, T)\right)$ that implies $v_{j} \in L^{2}\left(0, T ; H^{3}\left(0, \ell_{j}\right)\right)$. Applying Theorem 3.3 we obtain that $v_{j}=0$ for all $j=1, \cdots, N$ that contradicts the fact that $\|\underline{v}\|_{L^{2}\left(0, T ; \mathbb{L}^{2}(\mathcal{T})\right)}=1$.

Finally we obtain that (Obs) is valid for a solution $(\mathrm{KdVd})$ with $\left\|U_{0}\right\|_{H} \leq R$. We conclude as in the linear case.

Remark 3.5 We can observe that the semi-global character is given by the assumption $\left\|U_{0}\right\|_{H} \leq R$ which is necessary in our proof. Specifically it is used to show that $\left(\lambda^{n}\right)_{n \in \mathbb{N}}$ is bounded. An interesting open problem is the following: Is (KdVd is globally well-posed and globally exponentially stable?

\section{Stabilization when not all damped terms are activated}

It is known that to obtain exponential stability of a single $\mathrm{KdV}$ equation we only need to add a damped term if the length is critical $(L \in \mathcal{N})$ [17]. In the network case, more precisely in [1] Theorem 3.6, the authors consider damping terms $a_{j}$ applying on the critical lengths edges except at most on one edge.

Now we will prove Theorem 1.4 following closely Section 6 of [21] and [11]. First note that if (1.8) holds the energy of (KdVd) defined by (1.6) satisfies 


$$
\begin{gathered}
\frac{\mathrm{d}}{\mathrm{d} t} E(t) \leq-(2 \alpha-N)\left|u_{1}(t, 0)\right|^{2}-\sum_{j=1}^{N}\left|\partial_{x} u_{j}(t, 0)\right|^{2}-2 \sum_{j \in I} \int_{\text {supp } a_{j}} a_{j}\left|u_{j}\right|^{2} d x \\
+\sum_{j \in I} \int_{\omega_{j}}\left(b_{j}-\xi_{j}\right)\left|u_{j}\left(t-h_{j}, x\right)\right|^{2} d x+\sum_{j \in I} \int_{\omega_{j}}\left(b_{j}+\xi_{j}\right)\left|u_{j}\right|^{2} d x \\
+\sum_{j \in I^{*}} \int_{\omega_{j}}\left(-2 a_{j}(x)+b_{j}(x)+\xi_{j}(x)\right)\left|u_{j}(t, x)\right|^{2} d x-\sum_{j \in I^{*}} \int_{\left(0, \ell_{j}\right) / \omega_{j}} a_{j}(x)\left|u_{j}(t, x)\right|^{2} d x \\
+\sum_{j \in I^{*}} \int_{\omega_{j}}\left(b_{j}(x)-\xi_{j}(x)\right)\left|u_{j}\left(t-h_{j}, x\right)\right|^{2} d x .
\end{gathered}
$$

From the last inequality we can see that in this case the energy of the system $\mathrm{KdVd}$ is not decreasing in general, this by the action of the terms $b_{j}+\xi_{j}>0$ in $\omega_{j}$ for $j \in I$. Following [11] we consider the next auxiliary problem for which the energy will be decreasing. This system is close to $\mathrm{KdVd}$

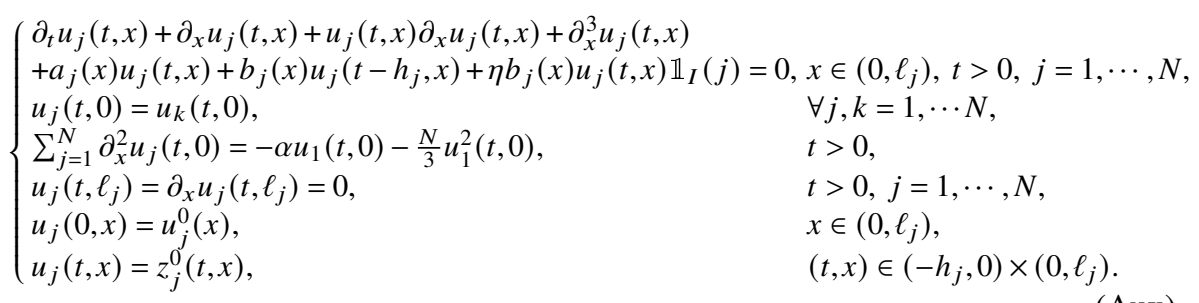

(Aux)

where $\mathbb{1}_{I}(j)$ is the indicator function of the set $I$ and $\eta>0$. Then we consider the energy (1.6) with $\xi_{j}=\eta b_{j}$ for $j \in I$, that is

$$
\begin{gathered}
E(t)=\sum_{j=1}^{N} \int_{0}^{\ell_{j}}\left|u_{j}\right|^{2} d x+\eta \sum_{j \in I} h_{j} \int_{\omega_{j}} \int_{0}^{1} b_{j}\left|u_{j}\left(t-h_{j} \rho, x\right)\right|^{2} d x d \rho \\
+\sum_{j \in I^{*}} h_{j} \int_{\omega_{j}} \int_{0}^{1} \xi_{j}\left|u_{j}\left(t-h_{j} \rho, x\right)\right|^{2} d x d \rho
\end{gathered}
$$

where in this case for all $j \in I^{*}, \xi_{j}$ is a non-negative function belonging to $L^{\infty}\left(0, \ell_{j}\right)$ such that $\operatorname{supp} \xi_{j}=\operatorname{supp} b_{j}=\omega_{j}$ and

$$
b_{j}(x)+c_{0} \leq \xi_{j}(x) \leq 2 a_{j}(x)-b_{j}(x)-c_{0}, \text { in } \omega_{j}, \text { for } j \in I^{*} .
$$

Easy calculations show us that if $\eta>1$, then 


$$
\begin{gathered}
\frac{\mathrm{d}}{\mathrm{d} t} E(t) \leq-(2 \alpha-N)\left|u_{1}(t, 0)\right|^{2}-\sum_{j=1}^{N}\left|\partial_{x} u_{j}(t, 0)\right|^{2}-2 \sum_{j \in I} \int_{\text {supp } a_{j}} a_{j}\left|u_{j}\right|^{2} d x \\
+\sum_{j \in I}(1-\eta) \int_{\omega_{j}} b_{j}\left|u_{j}\right|^{2} d x+\sum_{j \in I}(1-\eta) \int_{\omega_{j}} b_{j}\left|u_{j}\left(t-h_{j}\right)\right|^{2} d x \\
+\sum_{j \in I^{*}} \int_{\omega_{j}}\left(-2 a_{j}(x)+b_{j}(x)+\xi_{j}(x)\right)\left|u_{j}(t, x)\right|^{2} d x-\sum_{j \in I^{*}} \int_{\left(0, \ell_{j}\right) / \omega_{j}} a_{j}(x)\left|u_{j}(t, x)\right|^{2} d x \\
+\sum_{j \in I^{*}} \int_{\omega_{j}}\left(b_{j}(x)-\xi_{j}(x)\right)\left|u_{j}\left(t-h_{j}, x\right)\right|^{2} d x \leq 0 .
\end{gathered}
$$

The main idea to deal with the case when supp $b_{j} \not \subset$ supp $a_{j}$ is to show the exponential stability of the linearization around 0 of Aux via a Lyapunov function following Section 3.1 and then pass to (LKdVd) using a perturbation result.

More precisely we are going to use the following theorem.

Theorem 4.1 (Theorem 1.1, [16]) Let $X$ be a Banach space and let $A$ be a the infinitesimal generator of a $C_{0}$ semigroup $T(t)$ on $X$ satisfying $\|T(t)\| \leq M e^{\omega t}$. If $B$ is bounded linear operator on $X$, then $A+B$ is the infinitesimal generator of a $C_{0}$ semigroup $S(t)$ on $X$ satisfying $\|S(t)\| \leq M e^{(\omega+M\|B\|) t)}$.

Remark 4.1 As we we said before we use a Lyapunov approach for the auxiliary system, for that we expect that our result holds for $L<\frac{\sqrt{3}}{2} \pi, \alpha>n / 2$ and small initial data. Also observing Theorem 4.1 we must require that $\|\underline{b}\|_{\mathcal{L}^{\infty}(\mathcal{T})}$ is small enough.

We start by proving the well-posedness of the linearization of $(\mathrm{Aux})$ around 0 . We omitted the details because they are closely similar to Section 2 .

$$
\begin{cases}\partial_{t} u_{j}(t, x)+\partial_{x} u_{j}(t, x)+\partial_{x}^{3} u_{j}(t, x)+a_{j}(x) u_{j}(t, x) & \\ +b_{j}(x) u_{j}\left(t-h_{j}, x\right)+\eta b_{j}(x) u_{j}(t, x) \mathbb{1}_{I}(j)=0, & x \in\left(0, \ell_{j}\right), t>0, j=1, \cdots, N, \\ u_{j}(t, 0)=u_{k}(t, 0), & \forall j, k=1, \cdots N, \\ \sum_{j=1}^{N} \partial_{x}^{2} u_{j}(t, 0)=-\alpha u_{1}(t, 0), & t>0, \\ u_{j}\left(t, \ell_{j}\right)=\partial_{x} u_{j}\left(t, \ell_{j}\right)=0, & t>0, j=1, \cdots, N, \\ u_{j}(0, x)=u_{j}^{0}(x), & x \in\left(0, \ell_{j}\right), \\ u_{j}(t, x)=z_{j}^{0}(t, x), & (t, x) \in\left(-h_{j}, 0\right) \times\left(0, \ell_{j}\right) .\end{cases}
$$

We set again $z_{j}(t, \rho, x)=\left.u_{j}\right|_{\omega_{j}}\left(t-h_{j} \rho, x\right) x \in \omega_{j}, \rho \in(0,1)$. Note that in this case as $\xi_{j}=\eta b_{j}$ the inner product defined for $H$ in Section 2 becomes

$$
\begin{gathered}
\left\langle(\underline{\underline{u}}),\left(\frac{v}{\underline{y}}\right)\right\rangle_{H}=\sum_{j=1}^{N} \int_{0}^{\ell_{j}} u_{j}(x) v_{j}(x) d x+\eta \sum_{j \in I} h_{j} \int_{\omega_{j}} \int_{0}^{1} b_{j}(x) z_{j}(\rho, x) y_{j}(\rho, x) d \rho d x \\
+\sum_{j \in I^{*}} h_{j} \int_{\omega_{j}} \int_{0}^{1} \xi_{j}(x) z_{j}(\rho, x) y_{j}(\rho, x) d \rho .
\end{gathered}
$$


Then (LAux) can be written as

$$
\left\{\begin{array}{l}
U_{t}(t)=\mathcal{A}_{0} U(t), t>0 \\
U(0)=U_{0}
\end{array}\right.
$$

where, $U=\left(\frac{u}{z}\right), U_{0}=\left(\left.\underline{z}^{0}\right|_{\omega} \frac{\underline{u}^{0}}{(-\underline{h} \cdot, \cdot)}\right)$ and the operator $\mathcal{A}_{0}$ is defined by:

$$
\mathcal{A}_{0} U=\left(\begin{array}{c}
-\left(\mathrm{D}_{x}(\mathcal{T})+\mathrm{D}_{x}^{3}(\mathcal{T})\right) \underline{u}-\underline{a} \cdot * \underline{u}-\underline{b} \cdot * \underline{\tilde{z}}(1, \cdot)-\eta \underline{b}^{I} \cdot * \underline{u} \\
-\frac{1}{\underline{h}} \cdot * \mathrm{D}_{\rho}(\mathcal{T}) \underline{z}
\end{array}\right)
$$

in which

$$
\left(\underline{b}^{I}\right)_{j}=\left\{\begin{array}{c}
b_{j}, \quad j \in I \\
0, \quad j \in I^{*}
\end{array}\right.
$$

and $D\left(\mathcal{A}_{0}\right)=D(\mathcal{A})$.

Theorem 4.2 Assume $\underline{a}, \underline{b} \in \mathbb{L}^{\infty}(\mathcal{T})$ componentwise non-negative that satisfy 1.1 and (1.9]. Let $U_{0} \in H$ and $\eta>1$. Then there exist a unique solution $U \in C([0, \infty) ; H)$ of (4.3). Moreover if $U_{0} \in D(\mathcal{A})$ then $U$ is a classical solution and

$$
U \in C\left([0, \infty) ; D\left(\mathcal{A}_{0}\right)\right) \cap C^{1}([0, \infty) ; H) .
$$

Proof Let $U=\left(\frac{u}{\underline{z}}\right) \in D\left(\mathcal{A}_{0}\right)$, then

$$
\begin{gathered}
\left\langle\mathcal{A}_{0} U, U\right\rangle \leq\left(\frac{N}{2}-\alpha\right) u_{1}^{2}(0)-\frac{1}{2} \sum_{j=1}^{N}\left(\partial_{x} u_{j}(0)\right)^{2}-\sum_{j \in I} \int_{\operatorname{supp} a_{j}} a_{j}\left|u_{j}\right|^{2} d x \\
+\frac{1}{2} \sum_{j \in I}(1-\eta) \int_{\omega} b_{j}\left|u_{j}\right|^{2} d x+\frac{1}{2} \sum_{j \in I}(1-\eta) \int_{\omega} b_{j}\left|u_{j}\left(t-h_{j}\right)\right|^{2} d x \\
-\frac{1}{2} \sum_{j \in I^{*}} \int_{\left(0, \ell_{j}\right) / \omega_{j}} a_{j}(x)\left|u_{j}(t, x)\right|^{2} d x+\sum_{j \in I^{*}} \int_{\omega_{j}}\left(-a_{j}(x)+\frac{b_{j}(x)}{2}+\frac{\xi_{j}(x)}{2}\right)\left|u_{j}(t, x)\right|^{2} d x \\
+\frac{1}{2} \sum_{j \in I^{*}} \int_{\omega_{j}}\left(b_{j}(x)-\xi_{j}(x)\right)\left|u_{j}\left(t-h_{j}, x\right)\right|^{2} d x \leq 0 .
\end{gathered}
$$

thus $\mathcal{A}_{0}$ is dissipative. Moreover

$$
\begin{gathered}
\mathcal{A}_{0}^{*}(\underline{\underline{v}} \underline{\underline{y}})=\left(\begin{array}{c}
\left(\mathrm{D}_{x}(\mathcal{T})+\mathrm{D}_{x}^{3}(\mathcal{T})\right) \underline{v}-\underline{a} \cdot * \underline{v}+\eta \underline{b} \cdot * \underline{\tilde{y}}(0, \cdot)-\eta \underline{b}^{I} \cdot * \underline{v} \\
\frac{1}{h} \cdot * \mathrm{D}_{\rho}(\mathcal{T}) \underline{y}
\end{array}\right) \\
D\left(\mathcal{A}_{0}^{*}\right)=\left\{\left(\frac{\underline{v}}{\underline{y}}\right), \underline{v} \in\left(\prod_{j=1}^{N} H^{3}\left(0, \ell_{j}\right)\right) \cap \mathbb{H}_{e}^{1}(\mathcal{T}), \sum_{j=1}^{N} \frac{\mathrm{d}^{2} v_{j}}{\mathrm{~d} x^{2}}(0)=(\alpha-N) v_{1}(0),\right. \\
\partial_{x} v_{j}(0)=0, \forall j=1, \cdots, N, \underline{y} \in \prod_{j=1}^{N} L^{2}\left(H^{1}(0,1) \times w_{j}\right), y_{j}(1, x)=-\left.\frac{1}{\eta} v_{j}\right|_{\omega_{j}}(x) \\
\text { for } \left.j \in I \text { and } y_{j}(1, x)=-\left.\frac{b_{j}}{\xi_{j}} v_{j}\right|_{\omega_{j}}(x) \text { for } j \in I^{*}\right\} .
\end{gathered}
$$


Let $V=\left(\frac{\underline{v}}{\underline{y}}\right) \in D\left(\mathcal{A}_{0}^{*}\right)$, then

$$
\begin{gathered}
\left\langle\mathcal{A}_{0}^{*} V, V\right\rangle \leq-\frac{1}{2} \sum_{j=1}^{N}\left|\partial_{x} v_{j}\left(\ell_{j}\right)\right|^{2}+\left(\frac{N}{2}-\alpha\right) v_{1}^{2}(0)-\sum_{j=1}^{N} \int_{\operatorname{supp} a_{j}} a_{j}\left|v_{j}\right|^{2} d x \\
+\sum_{j=1}^{N} \int_{\omega_{j}}\left(-\frac{\eta}{2}+\frac{2}{2 \eta}\right) b_{j}(x) v_{j}^{2}(x) d x+\sum_{j \in I^{*}} \int_{\omega_{j}}\left(-a_{j}+\frac{\xi_{j}}{2}+\frac{b_{j}^{2}}{2 \xi_{j}}\right)\left|v_{j}\right|^{2} d x \\
-\sum_{j \in I^{*}} \int_{\left(0, \ell_{j}\right) \backslash \omega_{j}} a_{j}\left|v_{j}\right|^{2} d x-\frac{1}{2} \sum_{j \in I^{*}} \int_{\omega_{j}} \xi_{j}\left|y_{j}(0, x)\right|^{2} d x \leq 0
\end{gathered}
$$

thus $\mathcal{A}_{0}^{*}$ is dissipative.

Now to prove the exponential stability of (LAux we consider the following Lyapunov function:

$$
V(t)=E(t)+\mu_{1} V_{1}+\mu_{2} V_{2}
$$

where $\mu_{1}, \mu_{2}>0, E(t)$ is defined by (4.1), $V_{1}(t)$ defined in (3.1) and $V_{2}(t)$ is given by

$$
\begin{aligned}
V_{2}(t) & =\sum_{j \in I} h_{j} \int_{\omega_{j}} \int_{0}^{1}(1-\rho) b_{j}(x)\left|u_{j}\left(t-h_{j} \rho, x\right)\right|^{2} d x d \rho \\
& +\sum_{j \in I^{*}} h_{j} \int_{\omega_{j}} \int_{0}^{1}(1-\rho)\left|u_{j}\left(t-h_{j} \rho, x\right)\right|^{2} d x d \rho .
\end{aligned}
$$

Proposition 4.1 Assume $\underline{a}, \underline{b} \in \mathbb{L}^{\infty}(\mathcal{T})$ componentwise non-negative that satisfy 1.1 and (1.9). Let $\alpha>\frac{N}{2}, \eta>1$ and $\left(\ell_{j}\right)_{j=1}^{N} \subset(0,+\infty)$ such that $L<\frac{\sqrt{3}}{2} \pi$. Then for every $U_{0} \in H$, the energy of (LAux defined by 4.1) decays exponentially, that is, there exists $C>0, \gamma>0$ such that

$$
E(t) \leq C E(0) e^{-2 \gamma t}
$$

where

$$
\begin{gathered}
\gamma \leq \min \left\{\frac{\left(3 \mu_{1} \pi-\mu_{1} 4 L^{2}\right)}{8 L^{2}\left(1+L \mu_{1}\right)}, \min _{j \in I} \frac{\mu_{2}}{2 h_{j}\left(\eta+\mu_{2}\right)}, \min _{j \in I^{*}} \frac{\mu_{2}}{2 h_{j}\left(\xi_{j}+\mu_{2}\right)}\right\}, \\
C=\left(1+\max \left\{L \mu_{1}, \frac{\mu_{2}}{\eta}, \frac{\mu_{2}}{b_{0}}\right\}\right) .
\end{gathered}
$$

for $\mu_{1}$ and $\mu_{2}$ such that

$$
\begin{aligned}
0<\mu_{1}<\left\{1, \frac{\eta-1}{L}, \frac{1}{N}(2 \alpha-N), \min _{j \in I^{*}}\left\{\inf _{\omega_{j}} \frac{\xi_{j}-b_{j}}{L b_{j}}, \inf _{\omega_{j}} \frac{2 a_{j}-b_{j}-\xi_{j}}{L b_{j}}\right\}\right\} \\
0<\mu_{2}<\min \left\{\eta-1-L \mu_{1}, \min _{j \in I^{*}} 2 a_{j}-b_{j}-\xi_{j}-L \mu_{1} b_{j}\right\} .
\end{aligned}
$$


Proof Let $\underline{u}$ be a regular solution of $(\underline{\mathrm{LAux}})$ with $U_{0} \in D\left(\mathcal{A}_{0}\right)$. Clearly with this definition of $V(t)$ we have that

$$
E(t) \leq V(t) \leq\left(1+\max \left\{L \mu_{1}, \frac{\mu_{2}}{\eta}, \frac{\mu_{2}}{b_{0}}\right\}\right) E(t)
$$

Now, integrating by parts we get

$$
\begin{gathered}
\frac{\mathrm{d}}{\mathrm{d} t} E(t) \leq(N-2 \alpha)\left|u_{1}(t, 0)\right|^{2}-\sum_{j=1}^{N}\left|\partial_{x} u_{j}(t, 0)\right|^{2}-2 \sum_{j \in I} \int_{\operatorname{supp} a_{j}} a_{j}\left|u_{j}\right|^{2} d x \\
+(1-\eta) \sum_{j \in I} \int_{\omega_{j}} b_{j}\left|u_{j}\right|^{2} d x+(1-\eta) \sum_{j \in I} \int_{\omega_{j}} b_{j}\left|u_{j}\left(t-h_{j}, x\right)\right|^{2} d x \\
+\sum_{j \in I^{*}} \int_{\omega_{j}}\left(-2 a_{j}+b_{j}+\xi_{j}\right)\left|u_{j}\right|^{2} d x-\sum_{j \in I^{*}} \int_{\operatorname{supp} a_{j} \backslash \omega_{j}} a_{j}\left|u_{j}\right|^{2} d x \\
+\sum_{j \in I^{*}} \int_{\omega_{j}}\left(b_{j}-\xi_{j}\right)\left|u_{j}\left(t-h_{j}, x\right)\right|^{2} d x
\end{gathered}
$$

together with

$$
\begin{gathered}
\frac{\mathrm{d}}{\mathrm{d} t} V_{1}(t)=\sum_{j=1}^{N} \int_{0}^{\ell_{j}}\left|u_{j}\right|^{2} d x-3 \sum_{j=1}^{N} \int_{0}^{\ell_{j}}\left|\partial_{x} u_{j}\right|^{2} d x-2 \sum_{j=1}^{N} u_{1}(t, 0) \partial_{x} u_{j}(t, 0) \\
-2 \eta \sum_{j \in I} \int_{\omega_{j}} x b_{j}\left|u_{j}\right|^{2} d x-2 \sum_{j=1}^{N} \int_{\text {supp } a_{j}} x a_{j}\left|u_{j}\right|^{2} d x-2 \sum_{j=1}^{N} \int_{\omega_{j}} x b_{j} u_{j}(t, x) u_{j}\left(t-h_{j}, x\right) d x, \\
\frac{\mathrm{d}}{\mathrm{d} t} V_{2}(t)=\sum_{j \in I} \int_{\omega_{j}} b_{j}\left|u_{j}\right|^{2} d x+\sum_{j \in I^{*}} \int_{\omega_{j}}\left|u_{j}\right|^{2} d x-\sum_{j \in I} \int_{\omega_{j}} b_{j} \int_{0}^{1}\left|u_{j}\left(t-h_{j} \rho, x\right)\right|^{2} d \rho d x \\
-\sum_{j \in I^{*}} \int_{\omega_{j}} \int_{0}^{1}\left|u_{j}\left(t-h_{j} \rho, x\right)\right|^{2} d \rho d x .
\end{gathered}
$$
$\gamma>0$

Using integrations by parts and Poincaré's inequality, we can easily check that for

$$
\begin{gathered}
\frac{\mathrm{d}}{\mathrm{d} t} V(t)+2 \gamma V(t) \leq\left(N-2 \alpha+\mu_{1} N\right)\left|u_{1}(t, 0)\right|^{2}+\left(\mu_{1}-1\right) \sum_{j=1}^{N}\left|\partial_{x} u_{j}(t, 0)\right|^{2} \\
+\sum_{j \in I} \int_{\omega_{j}} b_{j}\left(1-\eta+\mu_{2}+\mu_{1} L\right)\left|u_{j}\right|^{2} d x+\sum_{j \in I} \int_{\omega_{j}} b_{j}\left(1-\eta+\mu_{1} L\right)\left|u_{j}\left(t-h_{j}, x\right)\right|^{2} d x \\
+\sum_{j \in I^{*}} \int_{\omega_{j}}\left(-2 a_{j}+b_{j}+\xi_{j}+\mu_{2}+L \mu_{1} b_{j}\right)\left|u_{j}\right|^{2} d x
\end{gathered}
$$




$$
\begin{gathered}
+\sum_{j \in I^{*}} \int_{\omega_{j}}\left(b_{j}-\xi_{j}+\mu_{1} L b_{j}\right)\left|u_{j}\left(t-h_{j}\right)\right|^{2} d x \\
+\sum_{j \in I} \int_{\omega_{j}} \int_{0}^{1}\left(2 \gamma h_{j}\left(\mu_{2}+\eta\right)-\mu_{2}\right)\left|u_{j}\left(t-h_{j} \rho, x\right)\right|^{2} d x \\
+\sum_{j \in I^{*}} \int_{\omega_{j}} \int_{0}^{1}\left(2 \gamma h_{j}\left(\mu_{2}+\xi_{j}\right)-\mu_{2}\right)\left|u_{j}\left(t-h_{j} \rho, x\right)\right|^{2} d x \\
+\left[\frac{4 L^{2}\left(\mu_{1}+2 \mu_{1} \gamma L+2 \gamma\right)}{\pi^{2}}-3 \mu_{1}\right] \sum_{j=1}^{N} \int_{0}^{\ell_{j}}\left|\partial_{x} u_{j}(t, x)\right|^{2} d x .
\end{gathered}
$$

Taking

$$
\begin{aligned}
0<\mu_{1}<\left\{1, \frac{\eta-1}{L}, \frac{1}{N}(2 \alpha-N), \min _{j \in I^{*}}\left\{\inf _{\omega_{j}} \frac{\xi_{j}-b_{j}}{L b_{j}}, \inf _{\omega_{j}} \frac{2 a_{j}-b_{j}-\xi_{j}}{L b_{j}}\right\}\right\} \\
0<\mu_{2}<\min \left\{\eta-1-L \mu_{1}, \min _{j \in I^{*}} 2 a_{j}-b_{j}-\xi_{j}-L \mu_{1} b_{j}\right\},
\end{aligned}
$$

and using that $L>\frac{\sqrt{3}}{2} \pi$ we can take

$$
\gamma \leq \min \left\{\frac{\left(3 \mu_{1} \pi-\mu_{1} 4 L^{2}\right)}{8 L^{2}\left(1+L \mu_{1}\right)}, \min _{j \in I} \frac{\mu_{2}}{2 h_{j}\left(\eta+\mu_{2}\right)}, \min _{j \in I^{*}} \frac{\mu_{2}}{2 h_{j}\left(\xi_{j}+\mu_{2}\right)}\right\} .
$$

With this $\frac{\mathrm{d}}{\mathrm{d} t} V(t)+2 \gamma V(t) \leq 0$ which implies

$$
E(t) \leq\left(1+\max \left\{L \mu_{1}, \frac{\mu_{2}}{\eta}, \frac{\mu_{2}}{b_{0}}\right\}\right) E_{0} e^{-2 \gamma t} .
$$

By density we can extend the result to $U_{0} \in H$.

Now we will obtain a stability result of (LKdVd) using a perturbation argument. Note first that the operator $\mathcal{A}$ introduced in Section 2 and associated with LKdVd) can be written as

$$
\mathcal{A}=\mathcal{A}_{0}+\mathcal{B},
$$

where $D(\mathcal{A})=D\left(\mathcal{A}_{0}\right)$ and $\mathcal{B}$ is the bounded operator on $H$ defined by

$$
\mathcal{B} U=\left(\begin{array}{c}
\eta \underline{b}^{I} \cdot * \underline{u} \\
\underline{0}
\end{array}\right), \quad U=(\underline{u} \underline{z}) \in H .
$$

Proposition 4.2 Assume $\underline{a}, \underline{b} \in \mathbb{L}^{\infty}(\mathcal{T})$ componentwise non-negative that satisfy (1.1) and (1.9). Let $\alpha>\frac{N}{2}, \eta>1$ and $\left(\ell_{j}\right)_{j=1}^{N} \subset(0,+\infty)$ such that $L<\frac{\sqrt{3}}{2} \pi$, then for every $U_{0} \in H$ there exists a unique mild solution $U \in C([0, \infty), H)$ for $(\mathrm{LKdVd}$. Moreover if $U_{0} \in D(\mathcal{A})$ then the solution is classical and $U \in C([0, \infty) ; D(\mathcal{A})) \cap C^{1}([0, \infty), H)$. Furthermore there exists $\delta=\delta(\alpha, \eta, L, \underline{h})>0$ such that if

$$
\|\underline{b}\|_{\mathbb{L}^{\infty}(\mathcal{T})} \leq \delta,
$$


then for every $U_{0} \in H$, the solution of (LKdVd) satisfies

$$
E(t) \leq C E(0) e^{-\gamma t}, \quad t>0,
$$

for $C, \gamma>0$ defined in Proposition 4.1.

Proof It is enough to apply Theorem 4.1. We note that $\|\mathcal{B}\| \leq \eta\|\underline{b}\|_{\mathbb{L}^{\infty}(\mathcal{T})}$ and then remark that

$$
-\frac{\gamma}{2}+\sqrt{C} \eta\|\underline{b}\|_{\mathbb{L}^{\infty}(\mathcal{T})}<0 \Longleftrightarrow\|\underline{b}\|_{\mathbb{L}^{\infty}(\mathcal{T})}<\frac{\gamma}{2 \eta \sqrt{C}}
$$

Finally, we obtain the local exponential stability for $(\mathrm{KdVd})$ in the case when $\operatorname{supp} b_{j} \not \subset$ supp $a_{j}$, for $j \in I^{*} \subset\{1, \cdots, N\}$ stated in Theorem 1.4

Proof of Theorem 1.4:

We just adapt the proof of Theorem 2.2 and Theorem 1.2 to obtain the exponential stability of the nonlinear case using the stability of (LAux) and small initial data.

\section{Numerical Simulations}

The purpose of this section is to illustrate the stabilization results obtained in this work. For that we are going to present some numerical simulations adapting the schemes used in [2, 5, 14]. We choose a final time $T$ and for simplicity we take $\ell_{j}=L$ and $a_{j}, b_{j}$ constant on their support for all $j=1, \cdots, N$. We build a uniform spatial and time discretization of $N_{x}+1$ and $N_{t}+1$ points respectively, separated by the steps $\Delta x=L / N_{x}$ and $\Delta t=T / N_{t}$. To deal with the delay term we choose the delay step $\Delta \rho=1 / N_{\rho}$. Now we introduce the notation $u_{j}(n \Delta t, i \Delta x)=u_{j, i}^{n}$ and $z_{j}(n \Delta t, k \Delta \rho, i \Delta x)=z_{j, i, k}^{n}$ for $i=0, \cdots, N_{x}, k=0, \cdots, N_{\rho}$ and $n=0, \cdots, N_{t}$. We use the following approximation for the derivatives:

$$
\mathrm{D}_{x}^{+} y_{i}=\frac{y_{i+1}-y_{i}}{\Delta x}, \quad \mathrm{D}_{x}^{-} y_{i}=\frac{y_{i}-y_{i-1}}{\Delta x}, \quad \mathrm{D}_{x} y_{i}=\frac{y_{i+1}-y_{i-1}}{2 \Delta x}, \quad \mathrm{D}_{\rho}^{+} e_{k}=\frac{e_{k+1}-e_{k}}{\Delta \rho} .
$$

In order to approximate the term of third order $\partial_{x}^{3}$ we use $\mathrm{D}_{x}^{+} \mathrm{D}_{x}^{+} \mathrm{D}_{x}^{-}$. Now, to consider the nonlinear terms we use explicit approximation $y_{i}^{n} \mathrm{D}_{x}^{+} y_{i}^{n}$ and for the nonlinear boundary condition we use a forward approximation for the second derivative which gives

$$
\left(\frac{1}{(\Delta x)^{2}}+\alpha\right) u_{j, 0}^{n+1}-\frac{2}{(\Delta x)^{2}} u_{j, 1}^{n+1}+\frac{1}{(\Delta x)^{3}} u_{j, 2}^{n+1}=-\frac{1}{3}\left(u_{j, 0}^{n}\right)^{2}, \quad j=1, \cdots, N, n=1 \cdots, N_{t} .
$$

Note now that by the boundary conditions we have that $u_{j, N_{x}}^{n}=u_{j, N_{x}-1}^{n}=0, u_{j, 0}^{n}=u_{k}^{n}$ for all $n=0, \cdot, N_{j}$ and $j, k=1 \cdots, N$. Now we define $I_{\omega_{j}}$, the set of index such that $i \in I_{\omega_{j}}$ if $i \Delta x \in \omega_{j}$. Then taking $C=\mathrm{D}_{x}^{+} \mathrm{D}_{x}^{+} \mathrm{D}_{x}^{-}+\mathrm{D}_{x}$ our scheme can be seen as 


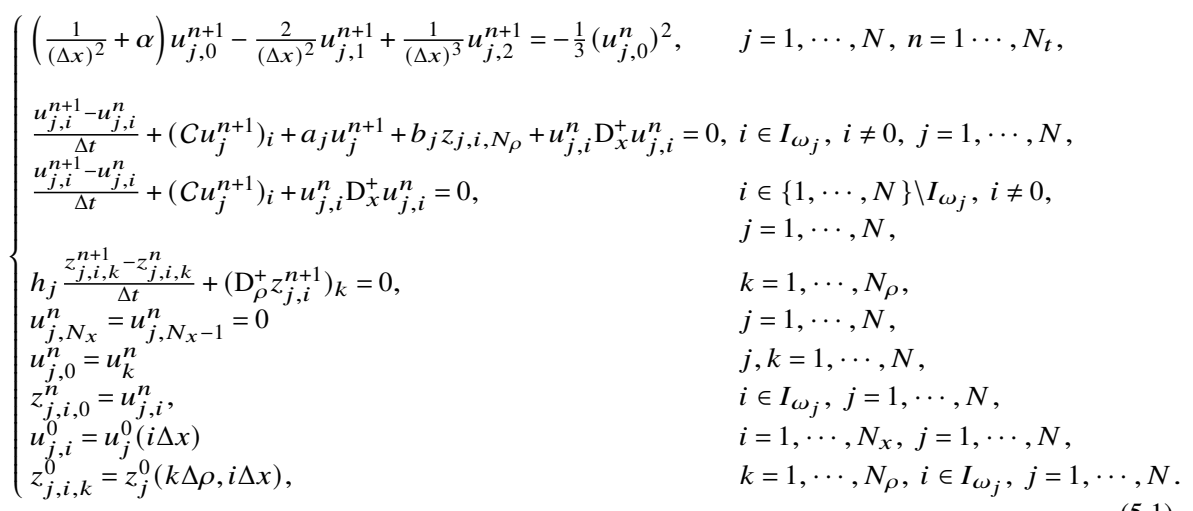

Now we use this scheme with the following parameters, $N=4, L=2$ and $\alpha=3$, for the discretization we use $N_{x}=100, N_{\rho}=100$, the initial conditions are $u_{j}^{0}=(1-\cos (2 \pi x / L))$ and $z_{j}^{0}=(1-\cos (2 \pi x / L)) \cos \left(2 \pi \rho h_{j}\right)$. As we say before we consider that the feedback terms are constant on their support, and we take $\omega_{1}=(0, L / 2), \omega_{2}=(0, L / 4), \omega_{3}=(0, L / 2)$ and $\omega_{4}=(0, L / 4)$.

For Figure 2 we use $T=5, N_{t}=100$ and delay $h_{1}=1, h_{2}=0.5, h_{3}=1$ and $h_{4}=1$. We can see that when there is not feedback terms $\left(a_{j}=b_{j}=0\right)$, the energy is exponentially decreasing and if we only activate the feedback term without delay, the energy decays more quickly. If we activate both feedback terms with and without delay the energy still decrease exponentially but slowly. Similar case happen if we not activate a feedback term without delay but we consider a feedback term with a small delay $\left(a_{4}=0, b_{j}=0.5\right)$. Finally if we consider only the action of delay feedback terms we can observe that in this case the energy decays very slowly.

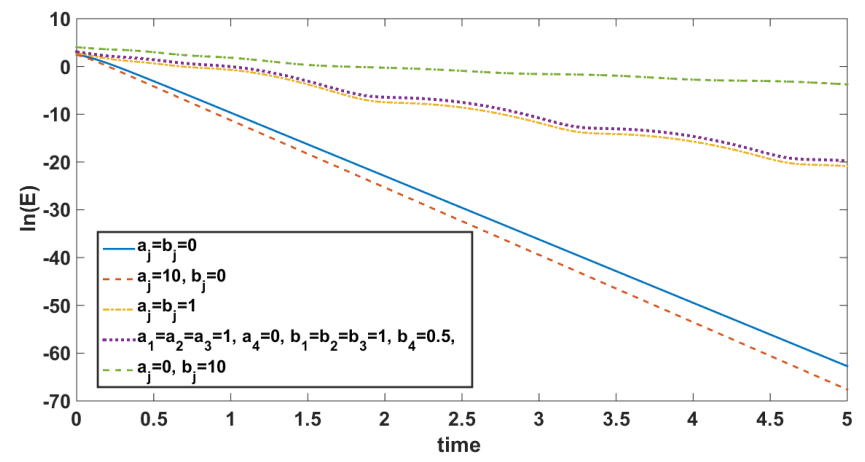

Fig. 2 Time-evolution of $t \mapsto \ln (E(t))$ for different values of feedback terms. 
For Figure 3 we use $T=10, N_{t}=200, a_{j}=0$ for $j=1, \cdots 4$. In this figure we can observe that in the case $a_{j}=0$ the energy decays exponentially if the feedback terms with delay are small enough. Also we can see that if the delay is bigger the feedback term with delay has to be smaller as written in Theorem 1.4

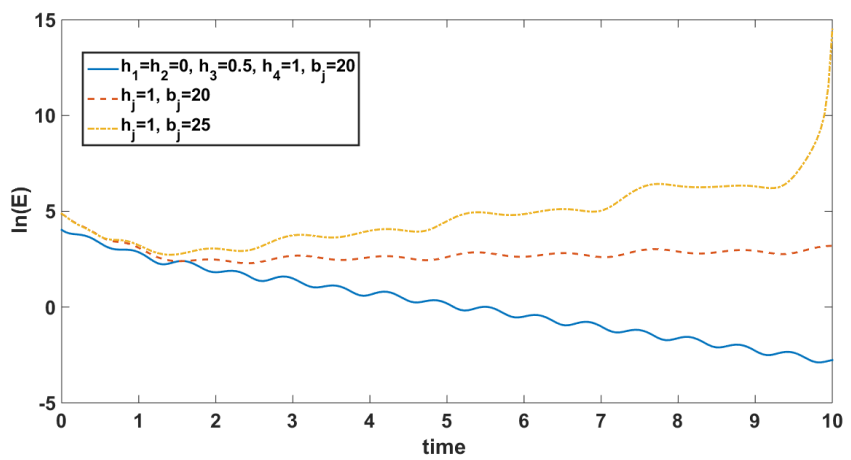

Fig. 3 Time-evolution of $t \mapsto \ln (E(t))$ for different values of feedback with delay term.

For Figure 4 we consider $T=2, N_{t}=100$, delays $h_{1}=0.1, h_{2}=0.2, h_{3}=0.3$ and $h_{4}=0.4$, feedback terms $a_{1}=2, a_{2}=4, a_{3}=6, a_{4}=8, b_{1}=0.5, b_{2}=1.5, b_{3}=2.5$ and $b_{4}=3.5$. We show $\ln (E(t))$ for different initial conditions $u_{j}^{0}=\theta(1-\cos (2 \pi x / L))$ and $z_{j}^{0}=\theta(1-\cos (2 \pi x / L)) \cos \left(2 \pi \rho h_{j}\right)$, for $\theta=0.5,2,5$. We show the graphics of $\ln \left(\frac{E(t)}{E(0)}\right)$ in order to normalize the energy. Here we can see that the decay rate does not seem to depend of the initial data.

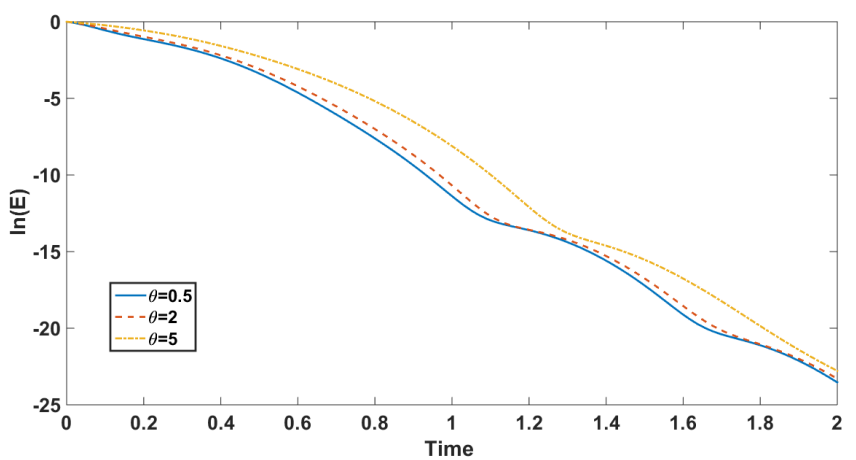

Fig. 4 Time-evolution of $t \mapsto \ln \left(\frac{E(t)}{E(0)}\right)$ for different values of $\theta>0$. 
To end we consider $T=2, N_{t}=100$, delays $h_{1}=0.1, h_{2}=0.2, h_{3}=0.3$ and $h_{4}=0.4$, feedback terms $a_{1}=2, a_{2}=4, a_{3}=6, a_{4}=8, b_{1}=0.5, b_{2}=1.5, b_{3}=2.5$ and $b_{4}=3.5$. We calculate the theoretical decay rate given by Theorem 1.1 we get $\ln (E(t)) \leq 2.9283-1.2143 \cdot 10^{-17} t$. Now we make a linear regression for the numerical obtained data and we get $\ln (E(t)) \approx 4.1129-12.766 t$. From here we can see that the theoretical decay rate given by Theorem 1.1 is much smaller than the one obtained numerically.

\section{Conclusions}

In this paper, was studied the well-posedness and exponential stability of a $\mathrm{KdV}$ equation on a Star Shaped Network with internal delayed feedback terms. The wellposedness was addressed including a new variable in order to take into account the delay and then studying the linearization around 0 of our system we obtain the local well-posedness for the nonlinear equation using the Banach fixed-point theorem.

First was considered the case where the support of delayed terms $b_{j}$ are included in the support of the feedback terms without delay $a_{j}$. In this was possible obtain the local exponential stability using a Lyapunov functional, this result holds for restricted lengths $L<\frac{\sqrt{3}}{2} \pi, \alpha>\frac{N}{2}$ and gives us an estimation of the decay rate. This estimation of the decay rate depends strongly on the Lyapunov Function used. Secondly using a contradiction argument an observability inequality for the linear system was derived that gives the exponential stability of the non linear system without restrictions on the lengths and $\alpha \geq \frac{N}{2}$. On a similar way working directly with the nonlinear system a semi-global stabilization result was obtained.

In the last stabilization results the case where non necessarily the support of delayed terms $b_{j}$ are included in the support of the feedback terms without delay $a_{j}$ has been considered. If this is the case and if the feedback delayed term $b_{j}$ is small enough, the local exponential stability for $L<\frac{\sqrt{3}}{2} \pi$ and $\alpha>\frac{N}{2}$ has been obtained.

Finally some numerical simulations have been presented. We showed how feedback delayed terms affects the stability (see Figure 2 and Figure 3). Also we observe that numerically in the case $a_{j}=0$ if the feedback delayed terms $b_{j}$ is big enough the system becomes unstable. Besides, we showed that the decay rate given by Theorem 1.1 is smaller than those obtained in simulations.

To conclude we present some open questions to be investigated:

1. As was said in Remark 3.1 the restriction on $L$ comes from the multiplier $x$ in $V_{1}$. Finding a new multiplier in order to obtain a result less restrictive is an open problem.

2. In this paper was considered that the delay acts internally. We are working on a delay term acting on the central node. 
3. The tools used in this work are inspired by [9]. For that a future research could be to study a KdV equation with a saturated control on a Star Shaped Network.

4. Typically the KdV equation is globally well-posedness in a bounded domain, the main difficulty to reach global well-posedness in the network case is Proposition 2.6 A global well-posedness of the KdV equation in a Star Shaped Network is an open problem.

5. In [7] a stabilization problem for the linear Kuramoto-Sivashinsky with delayed boundary control was studied. Studying a Kuramoto-Sivashinky equation on networks with or without delay is also a possible future work.

\section{References}

1. Ammari, K., Crépeau, E.: Feedback Stabilization and Boundary Controllability of the Kortewegde Vries Equation on a Star-Shaped Network. SIAM Journal on Control and Optimization 56(3), 1620-1639 (2018). DOI 10.1137/17M113959X. URL https://hal.archives-ouvertes.fr/ hal-02044236

2. Baudouin, L., Crépeau, E., Valein, J.: Two approaches for the stabilization of nonlinear KdV equation with boundary time-delay feedback. IEEE Transactions on Automatic Control 64(4), 1403-1414 (2019). DOI 10.1109/TAC.2018.2849564. URL https://hal .laas.fr/hal-01643321

3. Cerpa, E.: Control of a Korteweg-de Vries equation: a tutorial. Mathematical Control \& Related Fields 4(1), 45 (2014)

4. Cerpa, E., Crépeau, E., Moreno, C.: On the boundary controllability of the Korteweg-de Vries equation on a star-shaped network. IMA Journal of Mathematical Control and Information 37(1), 226-240 (2020)

5. Colin, T., Gisclon, M.: An initial-boundary-value problem that approximate the quarter-plane problem for the korteweg-de vries equation. Nonlinear Analysis: Theory, Methods \& Applications 46(6), 869-892 (2001). DOI 10.1016/s0362-546x(00)00155-3. URL https://doi .org/10.1016\% 2Fs0362-546x\%2800\%2900155-3

6. Datko, R.: Not all feedback stabilized hyperbolic systems are robust with respect to small time delays in their feedbacks. SIAM Journal on Control and Optimization 26(3), 697-713 (1988)

7. Guzmán, P., Marx, S., Cerpa, E.: Stabilization of the linear Kuramoto-Sivashinsky equation with a delayed boundary control. IFAC-PapersOnLine 52(2), 70-75 (2019)

8. Korteweg, D., de Vries, G.: On the change of form of long waves advancing in a rectangular channel, and a new type of long stationary wave. Phil. Mag 39, 422-443 (1895)

9. Marx, S., Cerpa, E., Prieur, C., Andrieu, V.: Global Stabilization of a Korteweg-de Vries Equation with saturating distributed control. SIAM Journal on Control and Optimization 55(3), 1452-1480 (2017)

10. Nicaise, S., Pignotti, C.: Stability and instability results of the wave equation with a delay term in the boundary or internal feedbacks. SIAM Journal on Control and Optimization 45(5), 1561-1585 (2006)

11. Nicaise, S., Pignotti, C.: Stabilization of second-order evolution equations with time delay. Mathematics of Control, Signals, and Systems 26(4), 563-588 (2014). DOI 10.1007/s00498-014-0130-1. URL https: //doi.org/10.1007\%2Fs00498-014-0130-1

12. Nicaise, S., Valein, J.: Stabilization of second order evolution equations with unbounded feedback with delay. ESAIM: Control, Optimisation and Calculus of Variations 16(2), 420-456 (2010)

13. Nicaise, S., Valein, J., Fridman, E.: Stability of the heat and of the wave equations with boundary time-varying delays. Discrete and Continuous Dynamical Systems Series S 2(3), 559 (2009)

14. Pazoto, A., Sepúlveda, M., Vera Villagran, O.: Uniform stabilization of numerical schemes for the critical generalized Korteweg-de Vries equation with damping. Numerische Mathematik 116, 317-356 (2010). DOI 10.1007/s00211-010-0291-X

15. Pazoto, A.F.: Unique continuation and decay for the Korteweg-de Vries equation with localized damping. ESAIM: Control, Optimisation and Calculus of Variations 11(3), 473-486 (2005)

16. Pazy, A.: Semigroups of linear operators and applications to partial differential equations, vol. 44 Springer Science \& Business Media (2012) 
17. Perla Menzala, G., Vasconcellos, C.F., Zuazua, E.: Stabilization of the Korteweg-de Vries equation with localized damping. Quarterly of Applied Mathematics 60(1), 111-129 (2002)

18. Rosier, L.: Exact boundary controllability for the Korteweg-de Vries equation on a bounded domain. ESAIM: Control, Optimisation and Calculus of Variations 2, 33-55 (1997). DOI 10.1051/cocv: 1997102. URL https://doi.org/10.1051\%2Fcocv\%3A1997102

19. Rosier, L., Zhang, B.Y.: Control and stabilization of the Korteweg-de Vries equation: recent progresses. Journal of Systems Science and Complexity 22(4), 647-682 (2009)

20. Saut, J.C., Scheurer, B.: Unique continuation for some evolution equations. Journal of differential equations 66(1), 118-139 (1987)

21. Valein, J.: On the asymptotic stability of the Korteweg-de Vries equation with time-delayed internal feedback (2021). URL https://hal.archives-ouvertes.fr/hal-02020757 Working paper or preprint

22. Zhang, B.Y.: Boundary stabilization of the Korteweg-de Vries equation. In: Control and estimation of distributed parameter systems: nonlinear phenomena, pp. 371-389. Springer (1994) 